DOI https://doi.org/10.24297/jam.v19i.9024

\title{
Four Bosons EM Conservation Laws
}

\author{
R. Doria ${ }^{1}$, I. Soares ${ }^{2}$ \\ ${ }^{1}$ Aprendanet, Petrópolis, Brazil,Quarks, Petrópolis, Brazil \\ ${ }^{2}$ CBPF, Rio de Janeiro, Brazil, Quarks Petrópolis, Brazil \\ ${ }^{1}$ doria@aprendanet.com.br, ${ }^{2}$ w.inacio@hotmail.com
}

\begin{abstract}
:
Electromagnetism is expressed from two basic postulates. They are light invariance and charge conservation. At this work one extends the Maxwell scenario from macroscopic to microscopic electromagnetism by following the elementary particles electric charge microscopic behavior. It yields a triune electric charge interrelationship. Three charges $\{+, 0,-\}$ be exchanged through a vector bosons quadruplet. It is called Four Bosons Electromagnetism.
\end{abstract}

A systemic EM physics appears to be understood. Maxwell photon is not enough for describing the microscopic electric charge physics. An extension for electromagnetic energy is obtained. The fields quadruplet $\left\{A_{\mu}, U_{\mu}, V_{\mu}^{ \pm}\right\}$are the porters of electromagnetic energy. They are the usual photon $A_{\mu}$, massive photon $U_{\mu}$ and two charged photons $V_{\mu}^{ \pm}$. A new understanding on EM phenomena has to be considered. A set determinism based on granular and collective fields is developed. A space-time evolution associated to a whole.

Conservation laws are studied. The EM phenomena is enlarged to three charges interchanges to $\{+, 0,-\}$. Two novelties appear. New features on nonlinear fields acting as own sources and on electric charge physics. Properties as conservation, conduction, transmission, interaction are extended to a systemic electromagnetism. A whole conservation law for electric charge emerges from three charges interwoven. Electric charge has a systemic behavior. Although there is no Coulomb law for zero electric charge, the Four Bosons Electromagnetism contains an EM energy which provides a neutral electromagnetism. Particles with zero charge $\left\{A_{\mu}, U_{\mu}\right\}$ are carrying EM energy.

Another consideration is on EM energy being transported by four nonlinear fields. A new physicality appears. The abelian nonlinearity generates fields charges. Fields are working as own sources through mass terms, trilinear and quadrilinear interactions, spin couplings. Consequently the photon is more than being a consequence from electric charge oscillations. It is able to generate its own charge. Introduce the meaning of photonics.

Thus, electric charge is no more the isolate electromagnetic source. There are another conservation laws. Fields sources appear through corresponding equations of motion, Bianchi identities, energy-momentum, Noether laws and angular momentum conservation laws. They move EM to a fields charges dependence. Together with electric charge they carry the electromagnetic flux. Supporting the Ahranov-Bohm experiment of potential fields as primitive entities.

\section{Introduction}

Electromagnetism phenomena was first observed through electric charge and magnets. During centuries we have been aggregating a more deep understanding on its meaning. From greek amber and magnets, electrostatic machines, condensers, batteries, the $18^{\text {th }}$ started to understand on charges and currents. At $19^{\text {th }}$ century, currents effects were studied by Oersted, Biot-Savart, Ampere, Ohm. A qualitative change happened for this first phase in 1831 when 
Faraday understood the existence of EM fields. From wires EM moved to invisible lines of force. This second phase takes form when Maxwell in 1864 wrote his equations in terms of fields as variables and charges as sources[1].

Maxwell equations express not only the relationship between charges and fields as a track for exploring fields as a physical entity. It preludes a physics beyond electric charge. It includes Faraday law and the displacement current. Given that, the $19^{\text {th }}$ century heritage was a field physics responsible for moving motors, energy distribution and light. Maxwell opened the door for something more than electric charge as source. For instance, it shows through Poynting vector that the EM flux does not follow the same direction of the electric charge current.

Nevertheless, a third EM phase is still being expected. Manifest an EM based on spin, nonlinear EM fields, photonics. There is an EM beyond Maxwell and QED to be discovered. A physics where fields generates fields. Current literature displays nearly 50 models beyond Maxwell [2]. Between them are 15 nonlinear models [3]. Most of them are effective theories indicating that a fundamental physics should exist. In this class of nonlinear models the Four Bosons Electromagnetism is being investigated [4].

A fundamental nonlinear EM is prospected. Its discovery was through the electric charge microscopic behaviour exposed by elementary particles. A nonlinear antireductionist abelian model which introduces four bosons quadruplet in order to promote the charge exchange $\Delta Q=0, \pm 1$. It performs a new relationship between charges and fields. It opens the possibility for understanding EM beyond electric charge. A physics where EM fields are acting as own sources.

Physics laws are not only understood by equations of motion and interaction but also through conservation laws. This microscopic electromagnetism flows based on the interchange between three charges $\{+, 0,-\}$ to be studied through four intermediate bosons $A_{\mu I} \equiv\left\{A_{\mu}, U_{\mu}, V_{\mu}^{ \pm}\right\}$. Interlaced nonlinear relationships between EM fields to be understood through conservation laws. $A_{\mu}$ means the usual photon, $U_{\mu}$ a massive photon, $V_{\mu}^{ \pm}$represent charged photons. Five types of conservation laws are obtained. They are from equations of motion, Bianchi identities, energy momentum tensor, Noether theorem, total momentum angular conservation. Their fluxes create sources depending on fields. They will show an EM flux beyond electric charge conservation and with the presence of potential fields as predicted by Aharanov-Bohm [5].

Our effort at this work is to explore the four bosons electromagnetism conservation laws. The understanding of this nonlinear behaviour beyond electric charge is a challenge to be analysed through conservation laws. At section 1, the Spin-Valued Four Bosons EM is rewritten [6]. Continuity equations are studied at sections 2 and 3. The energy momentum tensor is analysed at sections 5-9. At section 10, Noether conservation laws are considered. The angular momentum conservation law is observed at section 11. At conclusion, one discusses on perspectives from an EM moved by nonlinear fields.

\section{Lagrangian}

Electromagnetism contains a third element beyond charges and currents which is spin. Electron is also depending on spin- $\frac{1}{2}$ [7]. Stern-Gerlach experiment [8], Dirac equation [9] and spintronics [10] are showing the spin importance of EM phenomena. At this way through Four Bosons EM spin is incorporated as ab initio. It associates the four potentials responsible for EM energy transmissions to the Lorentz Group generators, $A_{\mu}^{I}=A_{\mu, \kappa, \lambda}^{I} \Sigma^{\kappa, \lambda}$. As consequence, from first principles, the subsequent set of nonlinear Maxwell equations will derive the interactions between magnetic moment and EM fields. 
The four bosons EM composes the fields set $\left\{A_{\mu}, U_{\mu}, V_{\mu}^{ \pm}\right\}$integrated into a common symmetry. The charge conservation interconnect them through an abelian gauge the $\mathrm{U}(1) \times \mathrm{SO}(2)_{\text {global }}$ symmetry. Considering the spin presence one associates each field to the Lorentz Group generator. It gives the following spin-valued Lagrangian

$$
\mathcal{L}^{\text {spin }}=\mathcal{L}_{K}^{\text {spin }}+\mathcal{L}_{M}^{\text {spin }}+\mathcal{L}_{I}^{\text {spin }}
$$

The kinect sector is

$$
\mathcal{L}_{K}^{\mathrm{spin}}=\mathcal{L}_{K}^{\mathrm{spin} A}+\mathcal{L}_{K}^{\mathrm{spin} S}
$$

where the antisymmetric part is

$$
\begin{aligned}
& \mathcal{L}_{K}^{\operatorname{spin} A}=\frac{1}{8}\left(\Sigma^{\mu \nu}\right)_{\sigma}^{\rho}\left(\Sigma^{\kappa \lambda}\right)_{\rho}^{\sigma} a_{1} F_{\mu \nu} F_{\kappa \lambda}+\frac{1}{4}\left(\Sigma^{\mu \kappa}\right)_{\sigma}^{\rho}\left(\Sigma^{\nu \lambda}\right)_{\rho}^{\sigma} a_{1} F_{\mu \nu} F_{\kappa \lambda}+ \\
& +\frac{1}{8}\left(\Sigma^{\mu \nu}\right)_{\sigma}^{\rho}\left(\Sigma^{\kappa \lambda}\right)_{\rho}^{\sigma} a_{2} U_{\mu \nu} U_{\kappa \lambda}+\frac{1}{4}\left(\Sigma^{\mu \kappa}\right)_{\sigma}^{\rho}\left(\Sigma^{\nu \lambda}\right)_{\rho}^{\sigma} a_{2} U_{\mu \nu} U_{\kappa \lambda}+ \\
& +\frac{1}{4}\left(\Sigma^{\mu \nu}\right)_{\sigma}^{\rho}\left(\Sigma^{\kappa \lambda}\right)_{\rho}^{\sigma} a_{3} V_{\mu \nu}^{+} V_{\kappa \lambda}^{-}+\frac{1}{2}\left(\Sigma^{\mu \kappa}\right)_{\sigma}^{\rho}\left(\Sigma^{\nu \lambda}\right)_{\rho}^{\sigma} a_{3} V_{\mu \nu}^{+} V_{\kappa \lambda}^{-}
\end{aligned}
$$

with

$$
\left(\Sigma_{\mu \nu}\right)_{\alpha \beta}=-i\left(g_{\mu \alpha} g_{\nu \beta}-g_{\mu \beta} g_{\nu \alpha}\right)
$$

which gives,

$$
\mathcal{L}_{K}^{\text {spin } A}=a_{1} F_{\mu \nu} F^{\mu \nu}+a_{2} U_{\mu \nu} U^{\mu \nu}+2 a_{3} V_{\mu \nu}^{+} V^{-\mu \nu}
$$

The symmetric sector is

$$
\begin{aligned}
& \mathcal{L}_{K}^{\operatorname{spin} S}=-\frac{1}{2}\left(\Sigma^{\mu \kappa}\right)_{\sigma}^{\rho}\left(\Sigma^{\nu \lambda}\right)_{\rho}^{\sigma} b_{(11)} S_{\mu \nu}^{1} S_{\kappa \lambda}^{1}-\frac{1}{2}\left(\Sigma^{\mu \kappa}\right)_{\sigma}^{\rho}\left(\Sigma^{\nu \lambda}\right)_{\rho}^{\sigma} b_{(22)} S_{\mu \nu}^{2} S_{\kappa \lambda}^{2}+ \\
& -\frac{1}{4}\left(\Sigma^{\mu \kappa}\right)_{\sigma}^{\rho}\left(\Sigma^{\nu \lambda}\right)_{\rho}^{\sigma} b_{(33)} S_{\mu \nu}^{+} S_{\kappa \lambda}^{-}-\frac{1}{24}\left(\Sigma_{\kappa \lambda}\right)_{\sigma}^{\rho}\left(\Sigma^{\kappa \lambda}\right)_{\rho}^{\sigma} c_{(11)} S_{\alpha}^{\alpha 1} S_{\beta}^{\beta 1}+ \\
& -\frac{1}{24}\left(\Sigma_{\kappa \lambda}\right)_{\sigma}^{\rho}\left(\Sigma^{\kappa \lambda}\right)_{\rho}^{\sigma} c_{(22)} S_{\alpha}^{\alpha 2} S_{\beta}^{\beta 2}-\frac{1}{12}\left(\Sigma_{\kappa \lambda}\right)_{\sigma}^{\rho}\left(\Sigma^{\kappa \lambda}\right)_{\rho}^{\sigma} c_{(12)} S_{\alpha}^{\alpha 1} S_{\beta}^{\beta 2}+ \\
& -\frac{1}{12}\left(\Sigma_{\kappa \lambda}\right)_{\sigma}^{\rho}\left(\Sigma^{\kappa \lambda}\right)_{\rho}^{\sigma} c_{(33)} S_{\alpha}^{\alpha+} S_{\beta}^{\beta-},
\end{aligned}
$$

which gives, 


$$
\mathcal{L}_{K}^{\operatorname{spin} S}=\mathcal{L}_{K}^{S}+\Delta_{K}^{S}
$$

where

$$
\begin{aligned}
& \mathcal{L}_{K}^{\operatorname{spin} S}=b_{(11)} S_{\mu \nu}^{1} S^{1 \mu \nu}+b_{(22)} S_{\mu \nu}^{2} S^{2 \mu \nu}+b_{(33)} S_{\mu \nu}^{+} S^{-\mu \nu}+ \\
& +\left(c_{(11)}-b_{(11)}\right) S_{\alpha}^{1 \alpha} S_{\beta}^{1 \beta}+c_{(12)} S_{\alpha}^{1 \alpha} S_{\beta}^{2 \beta}+ \\
& +\left(c_{(22)}-b_{(22)}\right) S_{\alpha}^{2 \alpha} S_{\beta}^{2 \beta}+\left(c_{(33)}-b_{(33)}\right) S_{\alpha}^{1 \alpha} S_{\beta}^{1 \beta}
\end{aligned}
$$

and

$$
\Delta_{K}^{S}=-b_{(11)} S_{\alpha}^{1 \alpha} S_{\beta}^{1 \beta}-b_{(22)} S_{\alpha}^{2 \alpha} S_{\beta}^{2 \beta}-b_{(33)} S_{\alpha}^{+\alpha} S_{\beta}^{-\beta}
$$

with the following definitions for the granular field strength tensors

$$
\begin{array}{lll}
F_{\mu \nu} \equiv \partial_{\mu} A_{\nu}-\partial_{\nu} A_{\mu}, & U_{\mu \nu} \equiv \partial_{\mu} U_{\nu}-\partial_{\nu} U_{\mu}, & V_{\mu \nu}^{ \pm} \equiv \partial_{\mu} V_{\nu}^{ \pm}-\partial_{\nu} V_{\mu}^{ \pm}, \\
S_{\mu \nu}^{1} \equiv \partial_{\mu} A_{\nu}+\partial_{\nu} A_{\mu}, & S_{\mu \nu}^{2} \equiv \partial_{\mu} U_{\nu}+\partial_{\nu} U_{\mu}, & S_{\mu \nu}^{ \pm} \equiv \partial_{\mu} V_{\nu}^{ \pm}+\partial_{\nu} V_{\mu}^{ \pm}
\end{array}
$$

The gauge fixing term $\mathcal{L}_{G F}^{\text {spin }}$ is

$$
\begin{aligned}
& \mathcal{L}_{G F}^{\mathrm{spin}}=-\frac{1}{96}\left(\Sigma_{\kappa \lambda}\right)_{\sigma}^{\rho}\left(\Sigma^{\kappa \lambda}\right)_{\rho}^{\sigma} \xi_{(11)} S_{\alpha}^{1 \alpha} S_{\beta}^{1 \beta}-\frac{1}{96}\left(\Sigma_{\kappa \lambda}\right)_{\sigma}^{\rho}\left(\Sigma^{\kappa \lambda}\right)_{\rho}^{\sigma} \xi_{(22)} S_{\alpha}^{2 \alpha} S_{\beta}^{2 \beta}+ \\
& -\frac{1}{48}\left(\Sigma_{\kappa \lambda}\right)_{\sigma}^{\rho}\left(\Sigma^{\kappa \lambda}\right)_{\rho}^{\sigma} \xi_{(12)} S_{\alpha}^{1 \alpha} S_{\beta}^{2 \beta}-\frac{1}{48}\left(\Sigma_{\kappa \lambda}\right)_{\sigma}^{\rho}\left(\Sigma^{\kappa \lambda}\right)_{\rho}^{\sigma} \xi_{(33)} S_{\alpha}^{+\alpha} S_{\beta}^{-\beta},
\end{aligned}
$$

or

$$
\mathcal{L}_{G F}^{\mathrm{spin}}=\frac{1}{4} \xi_{(11)} S_{\alpha}^{1 \alpha} S_{\beta}^{1 \beta}+\frac{1}{4} \xi_{(22)} S_{\alpha}^{2 \alpha} S_{\beta}^{2 \beta}+\frac{1}{2} \xi_{(12)} S_{\alpha}^{2 \alpha} S_{\beta}^{2 \beta}+\frac{1}{2} \xi_{(33)} S_{\alpha}^{+\alpha} S_{\beta}^{-\beta}
$$

The mass term $\mathcal{L}_{m}^{\text {spin }}$ is

$$
\begin{aligned}
& \mathcal{L}_{m}^{\text {spin }}=\frac{1}{48}\left(\Sigma_{\kappa \lambda}\right)_{\sigma}^{\rho}\left(\Sigma^{\kappa \lambda}\right)_{\rho}^{\sigma} \mu_{U}^{2} U_{\sigma} U^{\sigma}+\frac{1}{12}\left(\Sigma^{\alpha \lambda}\right)_{\sigma}^{\rho}\left(\Sigma_{\lambda}^{\beta}{ }_{\lambda}^{\sigma} \mu_{\rho}^{2} U_{\alpha} U_{\beta}+\right. \\
& +\frac{1}{24}\left(\Sigma_{\kappa \lambda}\right)_{\sigma}^{\rho}\left(\Sigma^{\kappa \lambda}\right)_{\rho}^{\sigma} \mu_{ \pm}^{2} V_{\sigma}^{+} V^{-\sigma}+\frac{1}{6}\left(\Sigma^{\alpha \lambda}\right)_{\sigma}^{\rho}\left(\Sigma_{\lambda}^{\beta}\right)_{\rho}^{\sigma} \mu_{ \pm}^{2} V_{\alpha}^{+} V_{\beta}^{-}
\end{aligned}
$$


which gives,

$$
\mathcal{L}_{M}=-\frac{1}{2} \mu_{U}^{2} U_{\sigma} U^{\sigma}-\mu_{ \pm}^{2} V_{\alpha}^{+} V_{\beta}^{-}
$$

The interacting sector is

$$
\mathcal{L}_{I}^{\text {spin }}=\mathcal{L}_{3}^{\text {spin }}+\mathcal{L}_{4}^{\text {spin }}
$$

The trilinear part is

$$
\mathcal{L}_{3}^{\text {spin }}=\mathcal{L}_{3}^{\text {spin A }}+\mathcal{L}_{3}^{\text {spin S }}+\mathcal{L}_{3}^{\text {spin st }}
$$

The trilinear antisymmetric term $\mathcal{L}_{3}^{\text {spin A }}$ is given by

$$
\begin{aligned}
& \mathcal{L}_{3}^{\operatorname{spin} A}=\left(\frac{1}{4}\left(\Sigma^{\mu \nu}\right)_{\sigma}^{\rho}\left(\Sigma^{\kappa \lambda}\right)_{\rho}^{\sigma}+\frac{1}{2}\left(\Sigma^{\mu \kappa}\right)_{\sigma}^{\rho}\left(\Sigma^{\nu \lambda}\right)_{\rho}^{\sigma}\right)\left(4 b_{1} F_{\mu \nu} \stackrel{[12]}{z}[\kappa \lambda]_{[\kappa]}+4 b_{2} U_{\mu \nu}{ }_{[}^{[12]}{ }_{[\kappa \lambda]}+\right. \\
& +4 b_{1} F_{\mu \nu} \stackrel{[+-]}{z}[\kappa \lambda]_{[\kappa \lambda]}+4 b_{2} U_{\mu \nu} \stackrel{[+--]}{z}_{[\kappa \lambda]}+4 \beta_{1} F_{\mu \nu} \stackrel{(+-)}{z}_{[\kappa \lambda]}+4 \beta_{2} U_{\mu \nu} \stackrel{(+-)}{z}_{[\kappa \lambda]}+ \\
& \left.+4 b_{3} V_{\mu \nu}^{+} \stackrel{[-1]}{z}_{[\kappa \lambda]}+4 b_{3} V_{\mu \nu}^{+} \stackrel{[-2]}{z}_{[\kappa \lambda]}+4 b_{3} V_{\mu \nu}^{-} \stackrel{[+1]}{z}_{[\kappa \lambda]}+4 b_{3} V_{\mu \nu}^{-} \stackrel{[+2]}{z}[\kappa \lambda]_{[\kappa \lambda}\right)
\end{aligned}
$$

Making the simplifications, we have

$$
\begin{aligned}
& \mathcal{L}_{3}^{\operatorname{spin} A}=4 b_{1} F_{\mu \nu}{ }^{[12]}[\mu \nu]+4 b_{2} U_{\mu \nu}{ }^{[12]}{ }^{[\mu \nu]}+4 b_{1} F_{\mu \nu}{ }^{[+-]^{[\mu \nu]}}+4 b_{2} U_{\mu \nu}{ }^{[+-]^{[\mu \nu]}}+ \\
& +4 \beta_{1} F_{\mu \nu}{ }^{(+-)}{ }^{[}[\mu \nu]+4 \beta_{2} U_{\mu \nu}{ }^{(+-)}{ }^{[\mu \nu]}+4 b_{3} V_{\mu \nu}^{+} \stackrel{[-1]}{z}[\mu \nu]+4 b_{3} V_{\mu \nu}^{+} \stackrel{[-2]}{z}[\mu \nu]+ \\
& \left.+4 b_{3} V_{\mu \nu}^{-}{ }^{[+11}\right]_{[\mu \nu]}+4 b_{3} V_{\mu \nu}^{-}{ }_{z}^{[+2]}[\mu \nu]
\end{aligned}
$$

At Appendix A the collective fields $z_{\mu \nu}$ are defined. 
The trilinear symmetric sector $\mathcal{L}_{3}^{\text {spin } S}$ is given by

$$
\begin{aligned}
& \mathcal{L}_{3}^{\operatorname{spin} S}=2 \beta_{1} S_{\mu \nu}^{1} \stackrel{(11)}{z} \mu \nu+4 \beta_{1} S_{\mu \nu}^{1} \stackrel{(12)}{z} \mu \nu+2 \beta_{1} S_{\mu \nu}^{1} \stackrel{(22)}{z} \mu \nu+4 \beta_{1} S_{\mu \nu}^{1}+{ }^{-3} \mu \nu+ \\
& +2 \beta_{2} S_{\mu \nu}^{2} \stackrel{(11)}{z} \mu \nu+4 \beta_{2} S_{\mu \nu}^{2} \stackrel{(12)}{z} \mu \nu+2 \beta_{2} S_{\mu \nu}^{2} \stackrel{(22)}{z} \mu \nu+4 \beta_{2} S_{\mu \nu}^{2}{ }^{+-3} \mu \nu+ \\
& +4 \beta_{3} S_{\mu \nu}^{+} \stackrel{(-1)}{z} \mu \nu+4 \beta_{3} S_{\mu \nu}^{+} \stackrel{(-2)}{z} \mu \nu+4 \beta_{3} S_{\mu \nu}^{-} \stackrel{(+1)}{z} \mu \nu+4 \beta_{3} S_{\mu \nu}^{-} \stackrel{(+2)}{z} \mu \nu+ \\
& +2\left(\rho_{1}-\beta_{1}\right) S_{\mu}^{\mu 1} \stackrel{(11)}{z} \underset{\nu}{\nu}+4\left(\rho_{1}-\beta_{1}\right) S_{\mu}^{\mu 1} \stackrel{(12)}{z}_{\nu}+ \\
& +2\left(\rho_{1}-\beta_{1}\right) S_{\mu}^{\mu 1} \stackrel{(22)}{z}{ }_{\nu}+4\left(\rho_{1}-\beta_{1}\right) S_{\mu}^{\mu 1}{ }_{z}^{+-3}{ }_{\nu}+ \\
& +2\left(\rho_{2}-\beta_{2}\right) S_{\mu}^{\mu 2} \stackrel{(11)}{z}_{\nu}+4\left(\rho_{2}-\beta_{2}\right) S_{\mu}^{\mu 2} \stackrel{(12)}{z}_{\nu}+ \\
& +2\left(\rho_{2}-\beta_{2}\right) S_{\mu}^{\mu 2} \stackrel{(22)}{z}_{\nu}+4\left(\rho_{2}-\beta_{2}\right) S_{\mu}^{\mu 2}{ }_{z}^{+-3}{ }_{\nu}+ \\
& +4\left(\rho_{3}-\beta_{3}\right) S_{\mu}^{\mu+}{ }_{\nu}^{(-1)}{ }_{\nu}+4\left(\rho_{3}-\beta_{3}\right) S_{\mu}^{\mu+}{ }_{\nu}^{(-2)}{ }_{\nu}+ \\
& +4\left(\rho_{3}-\beta_{3}\right) S_{\mu}^{\mu-} \stackrel{(+1)}{z}_{\nu}+4\left(\rho_{3}-\beta_{3}\right) S_{\mu}^{\mu-}{ }_{z}^{(+2)}{ }_{\nu}+ \\
& +2\left(\beta_{1}+4 \rho_{1}\right) S_{\mu}^{\mu 1} \stackrel{(11)}{\omega}_{\nu}+4\left(\beta_{1}+4 \rho_{1}\right) S_{\mu}^{\mu 1} \stackrel{(12)}{\omega}_{\nu}+ \\
& +2\left(\beta_{1}+4 \rho_{1}\right) S_{\mu}^{\mu 1} \stackrel{(22)}{\omega}_{\nu}+4\left(\beta_{1}+4 \rho_{1}\right) S_{\mu}^{\mu 1}{ }^{+-3}{ }_{\nu}+ \\
& +2\left(\beta_{2}+4 \rho_{2}\right) S_{\mu}^{\mu 2} \stackrel{(11)}{\omega}_{\nu}+4\left(\beta_{2}+4 \rho_{2}\right) S_{\mu}^{\mu 2} \stackrel{(12)}{\omega}_{\nu}+ \\
& +2\left(\beta_{2}+4 \rho_{2}\right) S_{\mu}^{\mu 2} \stackrel{(22)}{\omega}_{\nu}^{\nu}+4\left(\beta_{2}+4 \rho_{2}\right) S_{\mu}^{\mu 2}+\bar{\omega}_{\nu}{ }_{\nu}+ \\
& +4\left(\beta_{3}+4 \rho_{3}\right) S_{\mu}^{\mu+} \stackrel{(-1)}{\omega}_{\nu}+4\left(\beta_{3}+4 \rho_{3}\right) S_{\mu}^{\mu+}{ }_{\nu}^{(-2)}{ }_{\nu}+ \\
& +4\left(\beta_{3}+4 \rho_{3}\right) S_{\mu}^{\mu-} \stackrel{(+1)}{\omega}_{\nu}+4\left(\beta_{3}+4 \rho_{3}\right) S_{\mu}^{\mu-} \stackrel{(+2)}{\omega}_{\nu}
\end{aligned}
$$

The trilinear semitopological antisymmetric term $\mathcal{L}_{3}^{\text {spinst }}$, is

$$
\begin{aligned}
& \mathcal{L}_{3}^{\text {spinst }}=\left(-\frac{1}{24}\left(\Sigma^{\mu \nu}\right)_{\sigma}^{\rho}\left(\Sigma_{\mu \nu}\right)_{\rho}^{\sigma}\right) \epsilon^{\mu \nu \rho \sigma}\left\{2 i \stackrel{[1+]}{z}_{\mu \nu} V_{\rho \sigma}^{-}-2 i{ }_{z \nu}^{[1-]} V_{\rho \sigma}^{+}+\right. \\
& \left.+2 i \stackrel{[2+]}{z}_{\mu \nu} V_{\rho \sigma}^{-}-2 i \stackrel{[2-]}{z}_{\mu \nu} V_{\rho \sigma}^{+}+\left(\alpha_{0} F_{\rho \sigma}+\alpha_{1} U_{\rho \sigma}\right)\left[i\left({ }^{[+-]} z_{\mu \nu}+\stackrel{[-+]}{z}_{\mu \nu}\right)\right]+4 \stackrel{[12]}{z}_{\mu \nu}\left(\alpha_{0} F_{\rho \sigma}+\alpha_{1} U_{\rho \sigma}\right)\right\}
\end{aligned}
$$

where we have

$$
\begin{aligned}
& \stackrel{[1 \pm]}{z}_{\mu \nu}=\alpha_{0}\left(A_{\mu} V_{\nu}^{ \pm}-A_{\nu} V_{\mu}^{ \pm}\right) \\
& \stackrel{[2 \pm]}{z}_{\mu \nu}=\alpha_{1}\left(U_{\mu} V_{\nu}^{ \pm}-U_{\nu} V_{\mu}^{ \pm}\right)
\end{aligned}
$$

The quadratic sector is

$$
\mathcal{L}_{4}^{\text {spin }}=\mathcal{L}_{4}^{\text {spin } A}+\mathcal{L}_{4}^{\text {spin } S}+\mathcal{L}_{4}^{\text {spin } s t}
$$


The antisymmetric part $\mathcal{L}_{4}^{\text {spin } A}$ is

$$
\mathcal{L}_{4}^{\operatorname{spin} A}=\left(\frac{1}{4}\left(\Sigma^{\mu \nu}\right)_{\sigma}^{\rho}\left(\Sigma^{\kappa \lambda}\right)_{\rho}^{\sigma}+\frac{1}{2}\left(\Sigma^{\mu \kappa}\right)_{\sigma}^{\rho}\left(\Sigma^{\nu \lambda}\right)_{\rho}^{\sigma}\right) \mathcal{L}_{4}^{A}
$$

where the term $\mathcal{L}_{4}^{A}$ is defined in [?]. Simplifying, we have

$$
\begin{aligned}
& \mathcal{L}_{4}^{\text {spin } A}=\mathcal{L}_{4}^{A}+\Delta_{4}^{I A} \\
& \Delta_{4}^{I A}=0
\end{aligned}
$$


The symmetric part $\mathcal{L}_{4}^{\operatorname{spin} S}$ is

$$
\begin{aligned}
& \mathcal{L}_{4}^{\operatorname{spin} S}=\frac{1}{2}\left(\Sigma^{\mu \kappa}\right)_{\sigma}^{\rho}\left(\Sigma^{\nu \lambda}\right)_{\rho}^{\sigma}\left(2 \stackrel{[12]}{z}_{(\mu \nu)} \stackrel{[12]}{z}_{(\kappa \lambda)}+2 \stackrel{[12]}{z}_{(\mu \nu)} \stackrel{[21]}{z}_{(\kappa \lambda)}+4 \stackrel{[13+]}{z}_{(\mu \nu)}^{[13-]} z_{(\kappa \lambda)}+\right.
\end{aligned}
$$

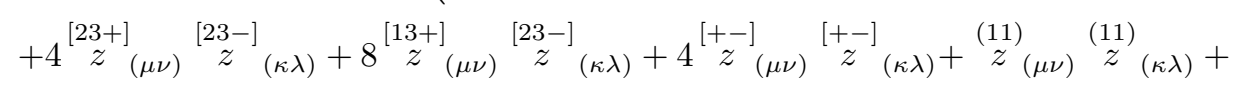

$$
\begin{aligned}
& +\stackrel{(22)}{z}_{(\mu \nu)} \stackrel{(22)}{z}_{(\kappa \lambda)}+2 \stackrel{(11)}{z}_{(\mu \nu)} \stackrel{(22)}{z}_{(\kappa \lambda)}+4 \stackrel{(12)}{z}_{(\mu \nu)} \stackrel{(12)}{z}_{(\kappa \lambda)}+2 \stackrel{(11)}{z}_{(\mu \nu)} \stackrel{(12)}{z}_{(\kappa \lambda)}+ \\
& +4 \stackrel{(22)}{z}_{(\mu \nu)} \stackrel{(12)}{z}_{(\kappa \lambda)}+4\left(\stackrel{(11)}{z}_{(\mu \nu)}+\stackrel{(22)}{z}_{(\mu \nu)}\right){ }^{+-3}{ }_{(\kappa \lambda)}+4 \stackrel{(11)}{\omega}_{(\mu \nu)} \stackrel{(11)}{\omega}_{(\kappa \lambda)}+ \\
& +4 \stackrel{(22)}{\omega}_{(\mu \nu)} \stackrel{(22)}{\omega}_{(\kappa \lambda)}+16 \stackrel{(11)}{\omega}_{(\mu \nu)} \stackrel{(12)}{\omega}_{(\kappa \lambda)}+16 \stackrel{(22)}{\omega}_{(\mu \nu)} \stackrel{(12)}{\omega}_{(\kappa \lambda)}+16 \stackrel{(12)}{\omega}_{(\mu \nu)} \stackrel{(12)}{\omega}_{(\kappa \lambda)}+ \\
& +2 \stackrel{(11)}{z}_{(\mu \nu)} \stackrel{(11)}{\omega}_{(\kappa \lambda)}+2 \stackrel{(22)}{z}_{(\mu \nu)} \stackrel{(22)}{\omega}_{(\kappa \lambda)}+8 \stackrel{(12)}{z}_{(\mu \nu)} \stackrel{(11)}{\omega}_{(\kappa \lambda)}+8 \stackrel{(12)}{z}_{(\mu \nu)} \stackrel{(22)}{\omega}_{(\kappa \lambda)}+
\end{aligned}
$$

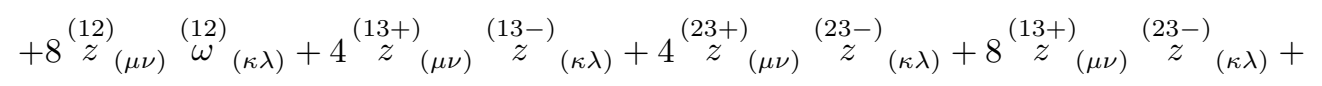

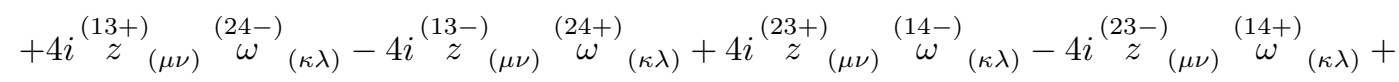

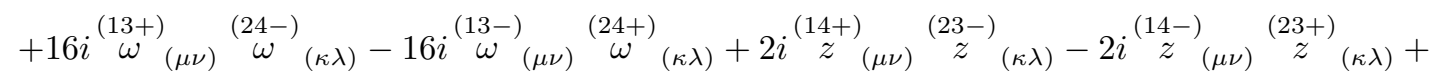

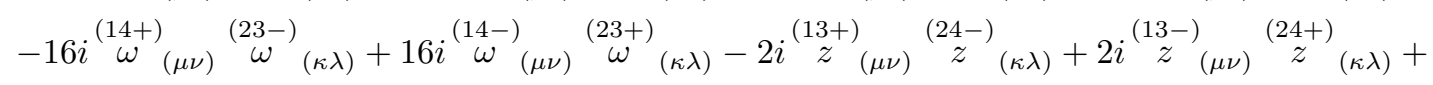

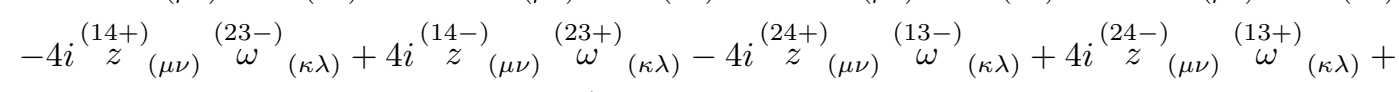

$$
\begin{aligned}
& \left.+8 \stackrel{(12)}{z}(\mu \nu)^{+-3}{ }_{(\kappa \lambda)}+4{ }^{+-3}{ }_{(\mu \nu)}+z_{(\kappa \lambda)}^{4}\right)+
\end{aligned}
$$

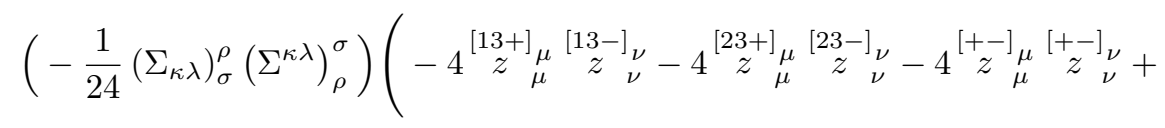

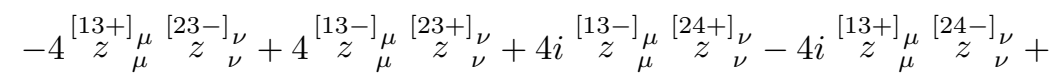

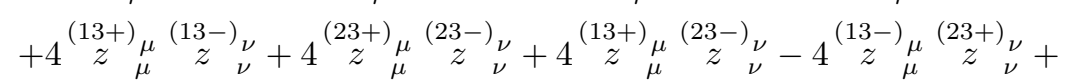

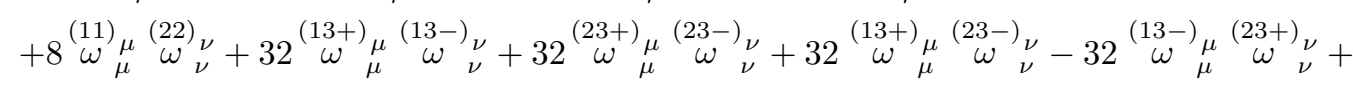

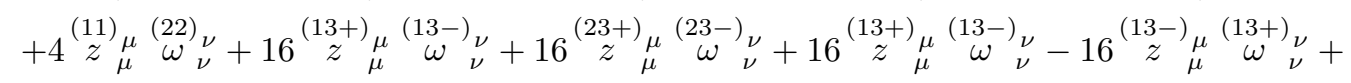

$$
\begin{aligned}
& +8\left\{\stackrel{(11)}{z}_{\mu}^{\mu}+\stackrel{(22)}{z}_{\mu}+2 \stackrel{(11)}{\omega}_{\mu}+2 \stackrel{(22)}{\omega}_{\mu}^{\mu}+2 \stackrel{(12)}{z}_{\mu}^{\mu}+4 \stackrel{(12)}{\omega}_{\mu}^{\mu}\right\}+{ }^{-3}{ }_{\nu}+ \\
& \left.-4{ }_{z}^{(+-)}{ }_{\mu}{ }_{z}^{(+-)}{ }_{\nu}+8+z_{\mu}^{+3}{ }_{\mu}^{+-4}{ }_{\nu}+16{ }^{+-3}{ }_{\mu}{ }^{+-}{ }^{-4}{ }_{\nu}\right)
\end{aligned}
$$


Using the relations in the previous section, we have

$$
\mathcal{L}_{4}^{\text {spin } S}=\mathcal{L}_{4}^{S}+\Delta_{4}^{S}
$$

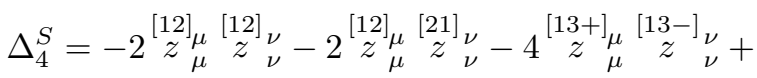

$$
\begin{aligned}
& -4{ }_{z}^{[23+]_{\mu}}{ }^{[23-]_{\nu}}{ }_{\nu}-8{ }^{[13+]_{\mu}}{ }_{\mu}^{[23-]_{\nu}}{ }_{\nu}-4{ }^{[+-]_{\mu}}{ }_{\mu}^{[+-]_{\nu}} \nu
\end{aligned}
$$

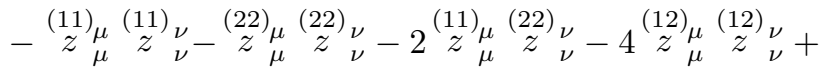

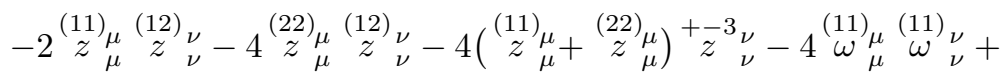

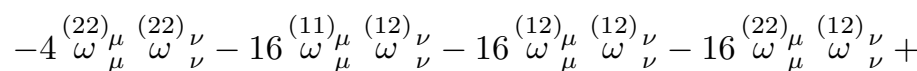

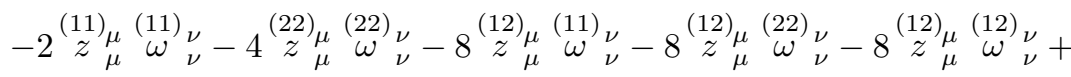

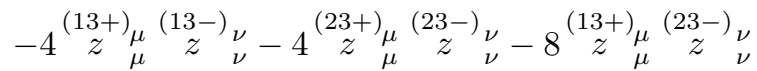

$$
\begin{aligned}
& \left.\left.\left.\left.-4 i{ }_{z}^{(13+)}\right)_{\mu} \stackrel{(24-)}{\omega}{ }_{\nu}+4 i{ }_{z}^{(13-)}\right)_{\mu} \stackrel{(24+)}{\omega}{ }_{\nu}-4 i{ }_{z}^{(23+)}\right)_{\mu} \stackrel{(14-)}{\omega}_{\nu}+4 i{ }_{z}^{(23-)}\right)_{\mu} \stackrel{(14+)}{\omega}{ }_{\nu}+
\end{aligned}
$$

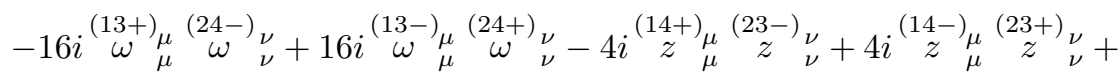

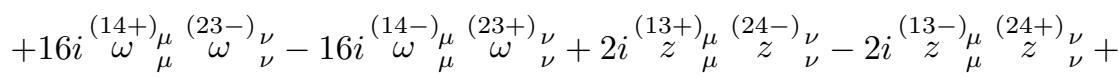

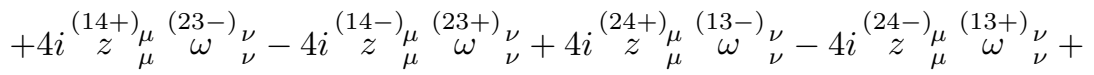

$$
\begin{aligned}
& -8{ }_{z}^{(12)}{ }_{\mu}+\bar{z}_{\nu}^{-3}{ }_{\nu}-4{ }_{z}^{+-3}{ }_{\mu}+\bar{z}_{\nu}{ }_{\nu}
\end{aligned}
$$

The semitopological part is $\mathcal{L}_{4}^{\text {spin st } S}$

$$
\begin{aligned}
& \mathcal{L}_{4}^{s t}=\left(-\frac{1}{24}\left(\Sigma^{\mu \nu}\right)_{\sigma}^{\rho}\left(\Sigma_{\mu \nu}\right)_{\rho}^{\sigma}\right) \epsilon^{\mu \nu \rho \sigma}\left\{44_{\mu \nu}^{[12]}{ }_{\rho \sigma}^{[12]}\right]_{\rho \sigma}+ \\
& \left.+4 \stackrel{[12]}{z}_{\mu \nu}\left(\stackrel{[+-]}{z}_{\rho \sigma}+\stackrel{[-+]}{z}_{\rho \sigma}\right)+\left(\stackrel{[+-]}{z}_{\mu \nu}+\stackrel{[-+]}{z}_{\mu \nu}\right)\left(\stackrel{[+-]}{z} \rho \sigma_{\rho \sigma}+{ }^{[-+]}{ }_{\rho \sigma}\right)\right\} .
\end{aligned}
$$

Summarizing, by taking fields Lorentz algebra valued, one derives a spin-valued Four Bosons Lagrangian

$$
\mathcal{L}^{\text {spin }}=\mathcal{L}_{\text {Four EM }}+\triangle_{\text {spin algebra valued }}
$$

where only trilinear and quadrilinear symmetric terms receive spin algebra valued contributions.

Thus, considering the three EM elements, which are electric charge, nonlinear fields and spin, it yields new Faraday lines of force getting through space. Currents flow and continuity equations are expressed. Two sets of conservation laws are inherent. They are the continuity equations and energy momentum tensor conservation. They will be studied at next chapters. 


\section{Conserved currents}

New sources are introduced to be understood through conservation laws. Given that electric charge physics should be understood from the interchange between positive, negative and zero charges, a new performance for transmitting EM energy happens throught a fields set $\left\{A_{\mu}, U_{\mu}, V_{\mu}^{ \pm}\right\}$. It includes new aspects as nonlinearity, spin, photonics.

Distinctly from QED [11], eq.(2.1) composes a system for electric charge transmission without external source. It yields a nonlinear electromagnetism where fields are their own charges and currents. While at QED the photon is just a messenger, a passive particle, eq.(2.1) derives a generic equation of motion by introducing nonlinear fields with a dependency beyond electric charge. It gives the generic expression

$$
\partial_{\nu}\left(F_{I}^{\nu \mu}+z_{I}^{\nu \mu}+g^{\mu \nu}\left(S_{\alpha I}^{\alpha}+z_{\alpha I}^{\alpha}\right)\right)-m_{I}^{2} A_{I}^{\mu}=J_{I}^{\mu}
$$

where $I$ is the flavour index. For the case, $I$ varies from 1 to $4 . F_{I}^{\nu \mu}$ and $z_{I}^{\nu \mu}$ are the corresponding granular and collective antisymmetric fields strengths; $S_{\alpha}^{\alpha I}$ and $z_{\alpha}^{\alpha}$ the corresponding granular and collective symmetric fields strengths; $m_{I}$ is the mass of the field $A_{I}^{\mu}$, and $J_{I}^{\mu}\left(A_{I}\right)$ are nonlinear currents depending on fields set $\left\{A_{I}^{\mu}\right\}$.

Eq.(3.1) can be decomposed on transverse and longitudinal sectors. Taking $A_{\mu I}^{T}=\Theta_{\mu \nu} A_{I}^{\nu}$ and $A_{\mu I}^{L}=\omega_{\mu \nu} A_{I}^{n u}$ where the transverse and longitudinal operators are $\Theta_{\mu \nu}=\eta_{\mu \nu}-\frac{\partial_{\mu} \partial_{\nu}}{\square}$ and $\omega_{\mu \nu}=\frac{\partial_{\mu} \partial_{\nu}}{\square}$

For spin-1 dynamics:

$$
\partial_{\nu}\left(F_{I}^{\nu \mu}+z_{I}^{[\nu \mu]}\right)-m_{I}^{2}\left(A_{I}^{\mu}\right)^{T}=\left(J_{I}^{\mu}\right)^{T}
$$

For spin-0 dynamics:

$$
\partial^{\mu}\left(S_{\alpha I}^{\alpha}+z_{\alpha I}^{\alpha}\right)-m_{I}^{2}\left(A_{I}^{\mu}\right)^{L}=\left(J_{I}^{\mu}\right)^{L}
$$

where these equations produce the following continuity equations

$$
\partial \cdot\left(m_{I}^{2}\left(A_{I}\right)^{T}-\left(J_{I}\right)^{T}\right)=0
$$

and

$$
\square\left(S_{\alpha I}^{\alpha}+z_{\alpha I}^{\alpha}\right)=\partial \cdot\left(J_{I}\right)^{L}
$$


Thus the quadruplet $A_{\mu I} \equiv\left\{A_{\mu}, U_{\mu}, V_{\mu}^{ \pm}\right\}$develops $(4+4)$ internal charges just depending on fields. They correspond to spin-1 and spin-0 fields. Notice that eq.(3.4) contains a dependence on mass term and on $\mathcal{L}_{3}^{I}$ and $\mathcal{L}_{4}^{I}$ through $J_{I}^{\mu}$. While eq.(3.5) introduce longitudinal sources depending on $\mathcal{L}_{K}^{S}, \mathcal{L}_{3}^{I}, \mathcal{L}_{4}^{I}$ and mass. They are showing a physics expliciting the presence of potential fields according to the expected physics beyond Maxwell [12].

A new photon appears which is no more an eletric charge consequence. It contains its own charge. The vectorial and longitudinal photons are distinguished by eqs.(3.2) and (3.3). Each of them contains its own source. A fact to consider a photonics without electric charge. As consequence photon-photon interaction beyond Breit-Wheeler [13], Adler [14], and Delbruck approaches [15].

\section{Bianchi identities}

Given the Bianchi collective antisymmetric identities

$$
\partial_{\mu} z_{[\nu \rho]}+\partial_{\rho} z_{[\mu \nu]}+\partial_{\nu} z_{[\rho \mu]}=\gamma_{[I J]} A_{\mu}^{I} F_{\rho \nu}^{J}+\gamma_{[I J]} A_{\nu}^{I} F_{\mu \rho}^{J}+\gamma_{[I J]} A_{\rho}^{I} F_{\nu \mu}^{J}
$$

which can be rewritten as

$$
\partial_{\mu} \tilde{z}^{[\mu \nu]}=j_{B}^{\nu}
$$

one gets the continuity equation

$$
\partial_{\nu} j_{B}^{\nu}=0
$$

Eqs.(4.2) and (4.3) are introducting a kind of collective fields monopole. Instead of Dirac puntiform magnetic charge [16] this compositon is more near to t'Hooft and Polyakov monopoles on Higgs fields [17]. It shows a collective fields charge based on the interaction between potential fields and electric and magnetic fields. Exposing a collective nonlinearity beyond Euler-Heisenberg [18] and Born-Infeld [19]. Eq.(4.1) introduces a Bianchi identity for the polarization and magnetization vectors [20].

\section{$5 \quad$ Energy-Momentum Tensor}

We should now analyze on the corresponding improved energy momentum tensor $\Theta_{\mu \nu}$. It follows the general conservation law [21]

$$
\partial_{\mu} \Theta^{\mu \nu}=0
$$

which is guaranteed by translation invariance Splitting in parts according to the Lagrangian, one gets 


$$
\Theta_{\mu \nu}=\Theta_{\mu \nu}^{K}+\Theta_{\mu \nu}^{M}+\Theta_{\mu \nu}^{G F}+\Theta_{\mu \nu}^{I}
$$

The kinect contribution is

$$
\Theta_{\mu \nu}^{K}=\Theta_{\mu \nu}^{A}+\Theta_{\mu \nu}^{S}
$$

where

$$
\begin{aligned}
& \Theta_{K \mu \nu}^{A}=\frac{1}{2} a_{1} F_{\alpha \beta} F^{\alpha \beta} g_{\mu \nu}-a_{1} F_{\mu \alpha} F_{\nu}^{\alpha}-a_{1} F_{\nu \alpha} F_{\mu}^{\alpha} \\
& +\frac{1}{2} a_{2} U_{\alpha \beta} U^{\alpha \beta} g_{\mu \nu}-a_{2} U_{\mu \alpha} U_{\nu}^{\alpha}-a_{2} U_{\nu \alpha} U_{\mu}^{\alpha} \\
& +a_{3} V_{\alpha \beta}^{-} V^{+\alpha \beta} g_{\mu \nu}-2 a_{3} V_{\mu \alpha}^{-} V_{\nu}^{+\alpha}-2 a_{3} V_{\nu \alpha}^{-} V_{\mu}^{+\alpha}
\end{aligned}
$$

and

$$
\begin{aligned}
& \Theta_{K \mu \nu}^{S}=\frac{1}{2} b_{11} S_{1 \alpha \beta} S_{1}^{\alpha \beta} g_{\mu \nu}-b_{11} S_{1 \mu \alpha} S_{1 \nu}^{\alpha}-b_{11} S_{1 \nu \alpha} S_{1 \mu}^{\alpha} \\
& +\frac{1}{2} b_{22} S_{2 \alpha \beta} S_{2}^{\alpha \beta} g_{\mu \nu}-b_{22} S_{2 \mu \alpha} S_{2 \nu}^{\alpha}-b_{22} S_{2 \alpha \nu} S 2_{\mu}^{\alpha}+ \\
& +b_{33} S_{\alpha \beta}^{-} S^{+\alpha \beta} g_{\mu \nu}-2 b_{33} S_{\mu \alpha}^{-} S_{\nu}^{+\alpha}-2 b_{33} S_{\nu \alpha}^{-} S_{\mu}^{+\alpha}+ \\
& \frac{1}{2} c_{11} S_{1 \alpha}^{\alpha} S_{1 \beta}^{\beta} g_{\mu \nu}-2 c_{11} S_{1 \mu \nu} S_{1 \alpha}^{\alpha}+ \\
& +\frac{1}{2} c_{22} S_{2 \alpha}^{\alpha} S_{2 \beta}^{\beta} g_{\mu \nu}-2 c_{22} S_{2 \mu \nu} S_{2 \alpha}^{\alpha}+ \\
& +c_{12} S_{1 \alpha}^{\alpha} S_{2 \beta}^{\beta} g_{\mu \nu}-2 c_{12} S_{1 \mu \nu} S_{2 \alpha}^{\alpha}-2 c_{12} S_{1 \alpha}^{\alpha} S_{2 \mu \nu}+ \\
& +c_{33} S_{\alpha}^{+\alpha} S_{\beta}^{-\beta} g_{\mu \nu}-2 c_{33} S_{\mu \nu}^{+} S_{\alpha}^{-\alpha}-2 c_{33} S_{\mu \nu}^{-} S_{\alpha}^{+\alpha}
\end{aligned}
$$

The mass term is

$$
\begin{aligned}
& \Theta_{\mu \nu}^{M}=-\frac{1}{4} \mu_{U}{ }^{2} U_{\alpha} U^{\alpha} g_{\mu \nu}+\frac{1}{2} \mu_{U}{ }^{2} U_{\mu} U_{\nu}+ \\
& -\frac{1}{2} \mu_{ \pm}{ }^{2} V_{\beta}^{-} V_{\beta}^{+} g_{\mu \nu}+\frac{1}{2} \mu_{ \pm}{ }^{2} V_{\mu}^{-} V_{\nu}^{+}+\frac{1}{2} \mu_{ \pm}{ }^{2} V_{\nu}^{-} V_{\mu}^{+}
\end{aligned}
$$

The gauge fixing term contribution is

$$
\begin{aligned}
& \Theta_{\mu \nu}^{G F}=\frac{1}{4} \xi_{11} S_{1 \alpha}^{\alpha} S_{1 \beta}^{\beta} g_{\mu \nu}-\frac{1}{2} \xi_{11} S_{1 \mu \nu} S_{1 \alpha}^{\alpha}+ \\
& +\frac{1}{4} \xi_{22} S_{2 \alpha}^{\alpha} S_{2 \beta}^{\beta} g_{\mu \nu}-\frac{1}{2} \xi_{22} S_{2 \mu \nu} S_{2 \alpha}^{\alpha}+ \\
& +\frac{1}{4} \xi_{12} S_{1 \alpha}^{\alpha} S_{2 \beta}^{\beta} g_{\mu \nu}-\frac{1}{2} \xi_{12} S_{1 \mu \nu} S_{2 \alpha}^{\alpha}-\frac{1}{2} \xi_{12} S_{1 \alpha}^{\alpha} S_{2 \mu \nu}+ \\
& +\frac{1}{4} \xi_{33} S_{\alpha}^{+\alpha} S_{\beta}^{-\beta} g_{\mu \nu}-\frac{1}{2} \xi_{33} S_{\mu \nu}^{+} S_{\alpha}^{-\alpha}-\frac{1}{2} \xi_{33} S_{\mu \nu}^{-} S_{\alpha}^{+\alpha}
\end{aligned}
$$

The interaction term is 


$$
\Theta_{\mu \nu}^{I}=\Theta_{\mu \nu}^{3 I}+\Theta_{\mu \nu}^{4 I}
$$

The trilinear contribution is

$$
\Theta_{\mu \nu}^{3 I}=\Theta_{\mu \nu}^{3 A}+\Theta_{\mu \nu}^{3 S}
$$

The trilinear antisymmetric contribution is

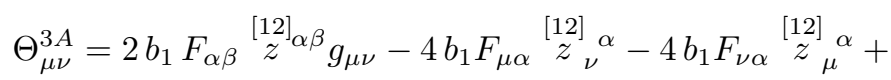

$$
\begin{aligned}
& +2 b_{2} U_{\alpha \beta}{ }^{[12]}{ }^{\alpha \beta} g_{\mu \nu}-4 b_{2} U_{\mu \alpha} \stackrel{[12]}{z}_{\nu}^{\alpha}-4 b_{2} U_{\nu \alpha} \stackrel{[12]}{z}_{\mu}^{\alpha}+
\end{aligned}
$$

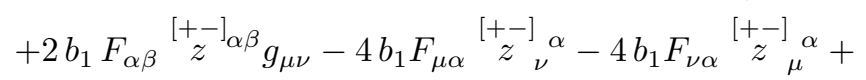

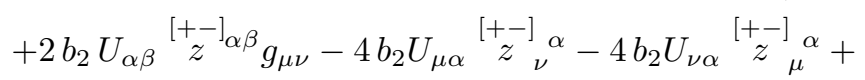

$$
\begin{aligned}
& +2 \beta_{1} F_{\alpha \beta}{ }^{(+-)}{ }^{+} \alpha \beta g_{\mu \nu}-4 \beta_{1} F_{\mu \alpha}{ }^{(+-)}{ }_{\nu}^{\alpha}-4 \beta_{1} F_{\nu \alpha}{ }^{(+-)}{ }_{\mu}^{\alpha}+ \\
& +2 \beta_{2} U_{\alpha \beta} \stackrel{(+-)}{z} \alpha \beta g_{\mu \nu}-4 \beta_{2} U_{\mu \alpha} \stackrel{(+-)}{z}_{\nu}^{\alpha}-4 \beta_{2} U_{\nu \alpha}{ }^{(+-)}{ }_{\mu}^{\alpha}+
\end{aligned}
$$

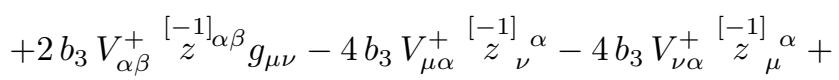

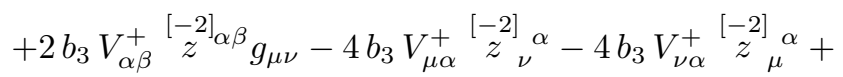

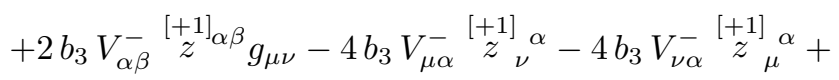

$$
\begin{aligned}
& +2 b_{3} V_{\alpha \beta}^{-}{ }_{z}^{[+2]} \alpha \beta g_{\mu \nu}-4 b_{3} V_{\mu \alpha}^{-}{ }_{z}^{[+2]}{ }_{\nu}^{\alpha}-4 b_{3} V_{\nu \alpha}^{-}{ }_{z}^{[+2]}{ }_{\mu}^{\alpha}
\end{aligned}
$$


The trilinear symmetric contribution is

$$
\begin{aligned}
& \Theta_{\mu \nu}^{3 S}=\beta_{1} S_{\alpha \beta}^{1} \stackrel{(11)}{z}{ }^{\alpha \beta} g_{\mu \nu}-2 \beta_{1} S_{\mu \alpha}^{1} \stackrel{(11)}{z}_{\nu}^{\alpha}-2 \beta_{1} S_{\nu \alpha}^{1} \stackrel{(11)}{z}{ }_{\mu}^{\alpha}+ \\
& +2 \beta_{1} S_{\alpha \beta}^{1} \stackrel{(12)}{z} \alpha \beta g_{\mu \nu}-4 \beta_{1} S_{\mu \alpha}^{1} \stackrel{(12)}{z}{ }_{\nu}^{\alpha}-4 \beta_{1} S_{\nu \alpha}^{1} \stackrel{(12)}{z}_{\mu}^{\alpha}+ \\
& +\beta_{1} S_{\alpha \beta}^{1} \stackrel{(22)}{z}{ }^{\alpha \beta} g_{\mu \nu}-2 \beta_{1} S_{\mu \alpha}^{1} \stackrel{(22)}{z}_{\nu}^{\alpha}-2 \beta_{1} S_{\nu \alpha}^{1} \stackrel{(22)}{z}_{\mu}^{\alpha}+ \\
& +\beta_{2} S_{\alpha \beta}^{2} \stackrel{(11)}{z} \alpha \beta g_{\mu \nu}-2 \beta_{2} S_{\mu \alpha}^{2} \stackrel{(11)}{z}_{\nu}^{\alpha}-2 \beta_{2} S_{\nu \alpha}^{2} \stackrel{(11)}{z}_{\mu}^{\alpha}+ \\
& +2 \beta_{2} S_{\alpha \beta}^{2} \stackrel{(12)}{z} \alpha \beta g_{\mu \nu}-4 \beta_{2} S_{\mu \alpha}^{2} \stackrel{(12)}{z}_{\nu}^{\alpha}-4 \beta_{2} S_{\nu \alpha}^{2} \stackrel{(12)}{z}_{\mu}^{\alpha}+ \\
& +\beta_{2} S_{\alpha \beta}^{2} \stackrel{(22)}{z} \alpha \beta g_{\mu \nu}-2 \beta_{2} S_{\mu \alpha}^{2} \stackrel{(22)}{z}_{\nu}^{\alpha}-2 \beta_{2} S_{\nu \alpha}^{2} \stackrel{(22)}{z}_{\mu}^{\alpha}+ \\
& +2 \beta_{3} S_{\alpha \beta}^{+}{ }^{(-1)}{ }^{\alpha \beta} g_{\mu \nu}-4 \beta_{3} S_{\mu \alpha}^{+} \stackrel{(-1)}{z}{ }_{\nu}^{\alpha}-4 \beta_{3} S_{\nu \alpha}^{+} \stackrel{(-1)}{z}_{\mu}^{\alpha}+ \\
& +2 \beta_{3} S_{\alpha \beta}^{+}{ }^{(-2)}{ }^{2 \beta} g_{\mu \nu}-4 \beta_{3} S_{\mu \alpha}^{+}{\stackrel{(-2)}{z}{ }_{\nu} \alpha}-4 \beta_{3} S_{\nu \alpha}^{+} \stackrel{(-2)}{z}_{\mu}^{\alpha}+ \\
& +2 \beta_{3} S_{\alpha \beta}^{-}{ }^{(+1)} \alpha \beta g_{\mu \nu}-4 \beta_{3} S_{\mu \alpha}^{-} \stackrel{(+1)}{z}_{\nu}^{\alpha}-4 \beta_{3} S_{\nu \alpha}^{-} \stackrel{(+1)}{z}_{\mu}^{\alpha}+ \\
& +2 \beta_{3} S_{\alpha \beta}^{-} \stackrel{(+2)}{z} \alpha \beta g_{\mu \nu}-4 \beta_{3} S_{\mu \alpha}^{-} \stackrel{(+2)}{z}_{\nu}^{\alpha}-4 \beta_{3} S_{\nu \alpha}^{+} \stackrel{(-1)}{z}_{\mu}^{\alpha}+ \\
& +2 \beta_{1} S_{\alpha \beta}^{1}{ }^{(+-3)}{ }^{(+\beta} g_{\mu \nu}-4 \beta_{1} S_{\mu \alpha}^{1}{ }^{(+-3)}{ }_{\nu}^{\alpha}-4 \beta_{1} S_{\nu \alpha}^{1}{ }^{(+-3)}{ }_{\mu}^{\alpha}+ \\
& +2 \beta_{2} S_{\alpha \beta}^{2}{ }^{(+-3)} z \beta g_{\mu \nu}-4 \beta_{2} S_{\mu \alpha}^{+}{ }^{(+-3)}{ }_{\nu}^{\alpha}-4 \beta_{2} S_{\nu \alpha}^{2}{ }^{(+-3)}{ }_{\mu}^{\alpha}+ \\
& +\rho_{1} S_{\alpha}^{1 \alpha}{\stackrel{(11)}{z}{ }_{\beta}}_{g_{\mu \nu}}-2 \rho_{1} S_{\mu \nu}^{1} \stackrel{(11)}{z}_{\alpha}-2 \rho_{1} S_{\alpha}^{1 \alpha} \stackrel{(11)}{z}_{\mu \nu}+ \\
& +2 \rho_{1} S_{\alpha}^{1 \alpha} \stackrel{(12)}{z}_{\beta} g_{\mu \nu}-4 \rho_{1} S_{\mu \nu}^{1} \stackrel{(12)}{z}_{\alpha}-4 \rho_{1} S_{\alpha}^{1 \alpha} \stackrel{(12)}{z}(\mu \nu)^{(12)}+ \\
& +\rho_{1} S_{\alpha}^{1 \alpha} \stackrel{(22)}{z}_{\beta} g_{\mu \nu}-2 \rho_{1} S_{\mu \nu}^{1} \stackrel{(22)}{z}_{\alpha}^{\alpha}-2 \rho_{1} S_{\alpha}^{1 \alpha} \stackrel{(22)}{z}_{\mu \nu}+ \\
& +\rho_{2} S_{\alpha}^{2 \alpha} \stackrel{(11)}{z}_{\beta} g_{\mu \nu}-2 \rho_{2} S_{\mu \nu}^{2} \stackrel{(11)}{z}_{\alpha}^{\alpha}-2 \rho_{2} S_{\alpha}^{2 \alpha} \stackrel{(11)}{z}_{\mu \nu}+ \\
& +2 \rho_{2} S_{\alpha}^{2 \alpha} \stackrel{(12)}{z}_{\beta} g_{\mu \nu}-4 \rho_{2} S_{\mu \nu}^{2} \stackrel{(12)}{z}_{\alpha}-4 \rho_{2} S_{\alpha}^{2 \alpha} \stackrel{(12)}{z}_{(\mu \nu)}+ \\
& +\rho_{2} S_{\alpha}^{2 \alpha} \stackrel{(22)}{z}_{\beta} g_{\mu \nu}-2 \rho_{2} S_{\mu \nu}^{2} \stackrel{(22)}{z}_{\alpha}^{\alpha}-2 \rho_{2} S_{\alpha}^{2 \alpha} \stackrel{(22)}{z}_{\mu \nu}+ \\
& +\left(\beta_{1}+4 \rho_{1}\right) S_{\alpha}^{1 \alpha} \stackrel{(11)}{\omega}_{\beta} g_{\mu \nu}-2\left(\beta_{1}+4 \rho_{1}\right) S_{\mu \nu}^{1} \stackrel{(11)}{\omega}_{\alpha}^{\alpha}-2\left(\beta_{1}+4 \rho_{1}\right) S_{\alpha}^{1 \alpha} \stackrel{(11)}{\omega}_{\mu \nu}+ \\
& +2\left(\beta_{1}+4 \rho_{1}\right) S_{\alpha}^{1 \alpha} \stackrel{(12)}{\omega}_{\beta} g_{\mu \nu}-4\left(\beta_{1}+4 \rho_{1}\right) S_{\mu \nu}^{1} \stackrel{(12)}{\omega}_{\alpha}^{\alpha}-4\left(\beta_{1}+4 \rho_{1}\right) S_{\alpha}^{1 \alpha} \stackrel{(12)}{\omega}_{(\mu \nu)}+ \\
& +\left(\beta_{1}+4 \rho_{1}\right) S_{\alpha}^{1 \alpha} \stackrel{(22)}{\omega}_{\beta} g_{\mu \nu}-2\left(\beta_{1}+4 \rho_{1}\right) S_{\mu \nu}^{1} \stackrel{(22)}{\omega}_{\alpha}^{\alpha}-2\left(\beta_{1}+4 \rho_{1}\right) S_{\alpha}^{1 \alpha} \stackrel{(22)}{\omega}_{\mu \nu}+ \\
& +\left(\beta_{2}+4 \rho_{2}\right) S_{\alpha}^{2 \alpha} \stackrel{(11)}{\omega}_{\beta} g_{\mu \nu}-2\left(\beta_{2}+4 \rho_{2}\right) S_{\mu \nu}^{2} \stackrel{(11)}{\omega}_{\alpha}^{\alpha}-2\left(\beta_{2}+4 \rho_{2}\right) S_{\alpha}^{2 \alpha} \stackrel{(11)}{\omega}_{\mu \nu}+ \\
& +2\left(\beta_{2}+4 \rho_{2}\right) S_{\alpha}^{2 \alpha} \stackrel{(12)}{\omega}_{\beta} g_{\mu \nu}-4\left(\beta_{2}+4 \rho_{2}\right) S_{\mu \nu}^{2} \stackrel{(12)}{\omega}_{\alpha}^{\alpha}-4\left(\beta_{2}+4 \rho_{2}\right) S_{\alpha}^{2 \alpha} \stackrel{(12)}{\omega}_{(\mu \nu)}+ \\
& +\left(\beta_{2}+4 \rho_{2}\right) S_{\alpha}^{2 \alpha} \stackrel{(22)}{\omega}_{\beta} g_{\mu \nu}-2\left(\beta_{2}+4 \rho_{2}\right) S_{\mu \nu}^{2} \stackrel{(22)}{\omega}_{\alpha}^{\alpha}-2\left(\beta_{2}+4 \rho_{2}\right) S_{\alpha}^{2 \alpha} \stackrel{(22)}{\omega}_{\mu \nu}+
\end{aligned}
$$




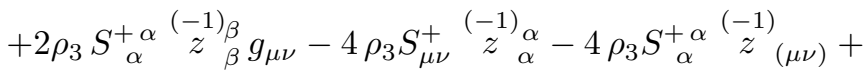

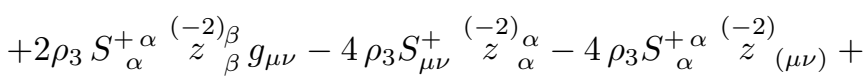

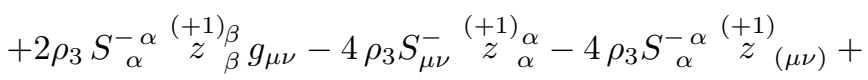

$$
\begin{aligned}
& +2 \rho_{3} S_{\alpha}^{-\alpha}{\stackrel{(+2)}{z}{ }_{\beta}}_{g_{\mu \nu}}-4 \rho_{3} S_{\mu \nu}^{-} \stackrel{(+2)}{z}_{\alpha}-4 \rho_{3} S_{\alpha}^{-\alpha} \stackrel{(+2)}{z}(\mu \nu)^{(-1)} \\
& +2\left(\beta_{3}+4 \rho_{3}\right) S_{\alpha}^{+\alpha} \stackrel{(-1)}{\omega}_{\beta} g_{\mu \nu}-4\left(\beta_{3}+4 \rho_{3}\right) S_{\mu \nu}^{+} \stackrel{(-1)}{\omega}_{\alpha}^{\alpha}-4\left(\beta_{3}+4 \rho_{3}\right) S_{\alpha}^{+\alpha} \stackrel{(-1)}{\omega}_{\mu \nu}+ \\
& +2\left(\beta_{3}+4 \rho_{3}\right) S_{\alpha}^{+\alpha} \stackrel{(-2)}{\omega}_{\beta} g_{\mu \nu}-4\left(\beta_{3}+4 \rho_{3}\right) S_{\mu \nu}^{+} \stackrel{(-2)}{\omega}_{\alpha}-4\left(\beta_{3}+4 \rho_{3}\right) S_{\alpha}^{+\alpha} \stackrel{(-2)}{\omega}_{\mu \nu}+ \\
& +2\left(\beta_{3}+4 \rho_{3}\right) S_{\alpha}^{-\alpha} \stackrel{(+1)}{\omega}_{\beta} g_{\mu \nu}-4\left(\beta_{3}+4 \rho_{3}\right) S_{\mu \nu}^{-} \stackrel{(+1)}{\omega}_{\alpha}-4\left(\beta_{3}+4 \rho_{3}\right) S_{\alpha}^{-\alpha} \stackrel{(+1)}{\omega}_{\mu \nu}+ \\
& +2\left(\beta_{3}+4 \rho_{3}\right) S_{\alpha}^{-\alpha} \stackrel{(+2)}{\omega}_{\beta} g_{\mu \nu}-4\left(\beta_{3}+4 \rho_{3}\right) S_{\mu \nu}^{-} \stackrel{(+2)}{\omega}_{\alpha}^{\alpha}-4\left(\beta_{3}+4 \rho_{3}\right) S_{\alpha}^{-\alpha} \stackrel{(+2)}{\omega}_{\mu \nu}+ \\
& +2 \rho_{1} S_{\alpha}^{1 \alpha}{ }^{+} \bar{z}_{\beta}^{3} g_{\mu \nu}-4 \rho_{1} S_{\mu \nu}^{1}{ }^{+{ }^{3}}{ }_{\alpha} \alpha-4 \rho_{1} S_{\alpha}^{1 \alpha}{ }_{z}^{+3}{ }_{\mu \nu}+ \\
& +2 \rho_{2} S_{\alpha}^{2 \alpha}{ }^{+-3}{ }_{\beta}^{3} g_{\mu \nu}-4 \rho_{2} S_{\mu \nu}^{2}{ }^{+}{ }^{-3}{ }_{\alpha}-4 \rho_{2} S_{\alpha}^{2 \alpha}{ }^{+}{ }^{-3}{ }_{\mu \nu}+ \\
& +2\left(\beta_{1}+4 \rho_{1}\right) S_{\alpha}^{1 \alpha}+{ }_{\omega}^{-3 \beta} g_{\mu \nu}-4\left(\beta_{1}+4 \rho_{1}\right) S_{\mu \nu}^{1}{ }^{+-3}{ }^{-3}{ }_{\alpha}-4\left(\beta_{1}+4 \rho_{1}\right) S_{\alpha}^{1 \alpha}{ }^{+}{ }^{-3}{ }_{\mu \nu}+ \\
& +2\left(\beta_{2}+4 \rho_{2}\right) S_{\alpha}^{2 \alpha}{ }^{+} \bar{\omega}_{\beta}^{-3} g_{\mu \nu}-4\left(\beta_{2}+4 \rho_{2}\right) S_{\mu \nu}^{2}{ }^{+}{ }^{-3}{ }_{\alpha}^{\alpha}-4\left(\beta_{2}+4 \rho_{2}\right) S_{\alpha}^{2 \alpha}{ }^{+}{ }^{-3}{ }_{\mu \nu}
\end{aligned}
$$

The four-linear contribution is

$$
\Theta_{\mu \nu}^{4 I}=\Theta_{\mu \nu}^{4 A}+\Theta_{\mu \nu}^{4 S}
$$

where the antisymmetric term is 


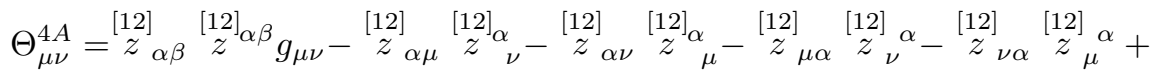

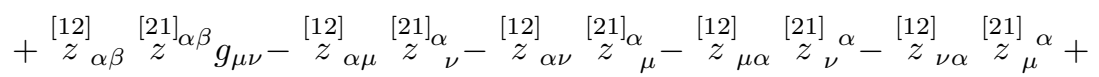

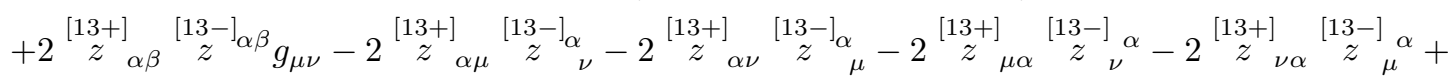

$$
\begin{aligned}
& +2 \stackrel{[23+]}{z}_{\alpha \beta} \stackrel{[23-]_{\alpha \beta}}{z} g_{\mu \nu}-2 \stackrel{[23+]}{z}_{\alpha \mu} \stackrel{[23-]_{\alpha}}{z}-2 \stackrel{[23+]}{z}_{\alpha \nu}^{[23-]_{\alpha}}{ }_{\mu}-2 \stackrel{[23+]}{z}_{\mu \alpha}^{[23-]} z_{\nu} \alpha-2 \stackrel{[23+]}{z}_{\nu \alpha}^{[23-]}{ }_{\mu}^{\alpha}+
\end{aligned}
$$

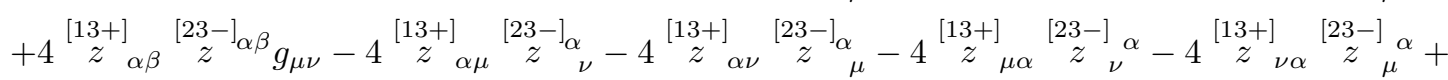

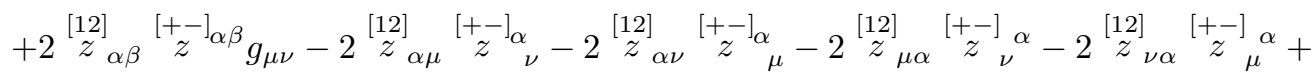

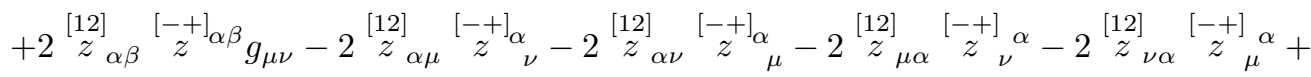

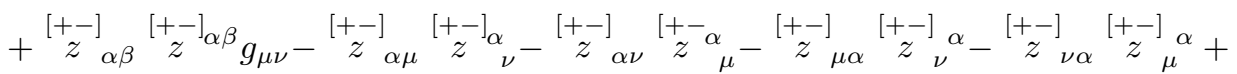

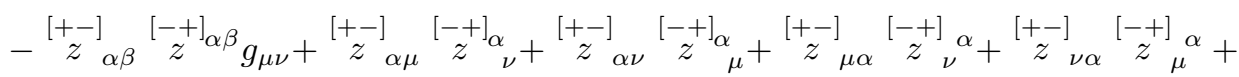

$$
\begin{aligned}
& -2{ }_{z}^{[13+]_{\alpha}}{ }_{z}^{[13-]_{\beta}} g_{\mu \nu}+4 \stackrel{[13+]}{z}(\mu \nu) \stackrel{[13-]_{\alpha}}{z}+4 \stackrel{[13-]}{z}(\mu \nu) \stackrel{[13+]_{\alpha}}{z}+
\end{aligned}
$$

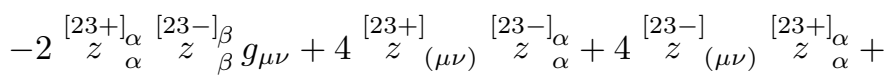

$$
\begin{aligned}
& -2{ }_{z}^{[13+]_{\alpha}}{ }_{z}^{[23-]_{\beta}} g_{\mu \nu}+4 \stackrel{[13+]}{z}(\mu \nu) \stackrel{[23-]_{\alpha}}{z}+4 \stackrel{[23-]}{z}(\mu \nu) \stackrel{[13+]_{\alpha}}{z}+ \\
& +2 \stackrel{[13-]_{\alpha}}{z}{ }_{z}^{[23+]_{\beta}} g_{\mu \nu}-4 \stackrel{[13-]}{z}(\mu \nu) \stackrel{[23+]_{\alpha}}{z}-4 \stackrel{[23+]}{z}(\mu \nu)_{z}^{[13-]_{\alpha}}+
\end{aligned}
$$

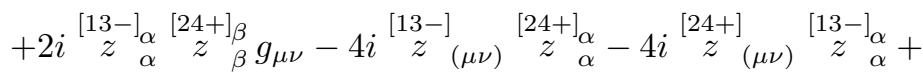

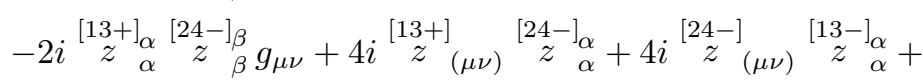

$$
\begin{aligned}
& -2{ }_{z}^{[+-]_{\alpha}}{ }^{[+-]_{\beta}} g_{\mu \nu}+4{ }_{z}^{[+-]}(\mu \nu){ }_{z}^{[+-]_{\alpha}}+4{ }_{z}^{[+-]}(\mu \nu){ }_{z}^{[+-]_{\alpha}}
\end{aligned}
$$

The symmetric contribution is 


$$
\begin{aligned}
& \Theta_{\mu \nu}^{4 S}=\frac{1}{2} \stackrel{(11)}{z}_{\alpha \beta} \stackrel{(11)}{z} \alpha \beta_{\mu \nu}-\stackrel{(11)}{z}_{\alpha \mu} \stackrel{(11)}{z}_{\nu} \alpha-\stackrel{(11)}{z}_{\alpha \nu} \stackrel{(11)}{z}_{\mu} \alpha+ \\
& +\frac{1}{2} \stackrel{(22)}{z}_{\alpha \beta} \stackrel{(22)}{z}_{\alpha \beta} g_{\mu \nu}-\stackrel{(22)}{z}_{\alpha \mu} \stackrel{(22)}{z}_{\nu}{ }^{\alpha}-\stackrel{(22)}{z}_{\alpha \nu} \stackrel{(22)}{z}_{\mu}^{\alpha}+ \\
& +\stackrel{(11)}{z}_{\alpha \beta} \stackrel{(11)}{\omega}^{\alpha \beta} g_{\mu \nu}-2 \stackrel{(11)}{\omega}_{\alpha \mu} \stackrel{(11)}{z}_{\nu} \alpha-2 \stackrel{(11)}{z}_{\alpha \nu} \stackrel{(11)}{\omega}_{\mu} \alpha+ \\
& +\stackrel{(22)}{z}_{\alpha \beta} \stackrel{(22)}{\omega}^{\alpha \beta} g_{\mu \nu}-2 \stackrel{(22)}{\omega}_{\alpha \mu} \stackrel{(22)}{z}_{\nu} \alpha-2 \stackrel{(22)}{z}_{\alpha \nu} \stackrel{(22)}{\omega}_{\mu} \alpha+ \\
& +2 \stackrel{(11)}{\omega}_{\alpha \beta} \stackrel{(11)}{\omega}^{\alpha \beta} g_{\mu \nu}-4 \stackrel{(11)}{\omega}_{\alpha \mu} \stackrel{(11)}{\omega}_{\nu} \alpha-4 \stackrel{(11)}{\omega}_{\alpha \nu} \stackrel{(11)}{\omega}_{\mu} \alpha+ \\
& +2 \stackrel{(22)}{\omega}_{\alpha \beta} \stackrel{(22)}{\omega}^{\alpha \beta} g_{\mu \nu}-4 \stackrel{(22)}{\omega}_{\alpha \mu} \stackrel{(22)}{\omega}_{\nu} \alpha-4 \stackrel{(22)}{\omega}_{\alpha \nu} \stackrel{(22)}{\omega}_{\mu} \alpha+
\end{aligned}
$$

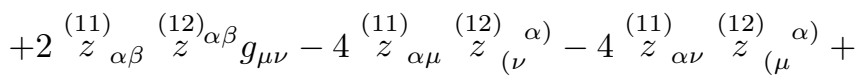

$$
\begin{aligned}
& +2 \stackrel{(22)}{z}_{\alpha \beta} \stackrel{(12)}{z} \alpha \beta g_{\mu \nu}-4 \stackrel{(22)}{z}_{\alpha \mu} \stackrel{(12)}{z}_{(\nu}^{\alpha)}-4 \stackrel{(22)}{z}_{\alpha \nu} \stackrel{(12)}{\omega}_{(\mu}^{\alpha)}+
\end{aligned}
$$

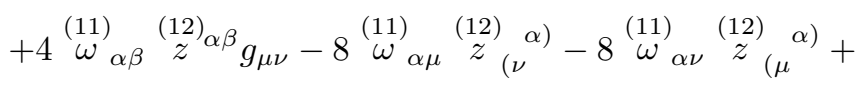

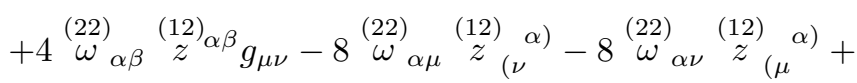

$$
\begin{aligned}
& +8 \stackrel{(11)}{\omega}_{\alpha \beta} \stackrel{(12)}{\omega}^{\alpha \beta} g_{\mu \nu}-16 \stackrel{(11)}{\omega}_{\alpha \mu} \stackrel{(12)}{\omega}_{(\nu}^{\alpha)}-16 \stackrel{(11)}{\omega}_{\alpha \nu} \stackrel{(12)}{\omega}_{(\mu}^{\alpha)}+ \\
& +8 \stackrel{(22)}{\omega}_{\alpha \beta} \stackrel{(12)}{\omega}^{\alpha \beta} g_{\mu \nu}-16 \stackrel{(22)}{\omega}_{\alpha \mu} \stackrel{(12)}{\omega}_{(\nu}^{\alpha)}-8 \stackrel{(22)}{\omega}_{\alpha \nu} \stackrel{(12)}{\omega}_{(\mu}^{\alpha)}+ \\
& +\stackrel{(22)}{z}_{\alpha \beta} \stackrel{(11)}{z}^{\alpha \beta} g_{\mu \nu}-2 \stackrel{(11)}{z}_{\alpha \mu} \stackrel{(11)}{z}_{\nu} \alpha-2 \stackrel{(22)}{z}_{\alpha \nu} \stackrel{(11)}{z}_{\mu} \alpha+ \\
& +\stackrel{(12)}{z}_{\alpha \beta} \stackrel{(12)}{z} \alpha \beta_{g_{\mu \nu}}-\stackrel{(12)}{z}_{\alpha \mu} \stackrel{(12)}{z}_{\nu}-\stackrel{(12)}{z}_{\alpha \nu} \stackrel{(12)}{z}_{\mu}-\stackrel{(12)}{z}_{\mu \alpha} \stackrel{(12)}{z}_{\nu} \alpha_{-} \stackrel{(12)}{z}_{\nu \alpha} \stackrel{(12)}{z}_{\mu} \alpha+ \\
& +4 \stackrel{(12)}{\omega}_{\beta \alpha} \stackrel{(12)}{z}_{\alpha \beta} g_{\mu \nu}-4 \stackrel{(12)}{z}_{\alpha \mu} \stackrel{(12)}{\omega}_{\nu} \alpha-4 \stackrel{(12)}{z}_{\alpha \nu} \stackrel{(12)}{\omega}_{\mu} \alpha-4 \stackrel{(12)}{z}_{\mu \alpha} \stackrel{(12)}{\omega}_{\alpha}{ }_{\nu}-4 \stackrel{(12)}{z}_{\nu \alpha} \stackrel{(12)}{\omega}_{\mu}+ \\
& +8 \stackrel{(12)}{\omega}_{\beta \alpha} \stackrel{(12)}{\omega}^{\alpha \beta} g_{\mu \nu}-8 \stackrel{(12)}{\omega}_{\alpha \mu} \stackrel{(12)}{\omega}_{\nu} \alpha-8 \stackrel{(12)}{\omega}_{\alpha \nu} \stackrel{(12)}{\omega}_{\mu} \alpha-8 \stackrel{(12)}{\omega}_{\mu \alpha} \stackrel{(12)}{\omega}_{\alpha}{ }_{\nu}-8 \stackrel{(12)}{\omega}_{\nu \alpha} \stackrel{(12)}{\omega}_{\alpha}{ }_{\mu}+ \\
& +\stackrel{(12)}{z}_{\beta \alpha} \stackrel{(12)}{z} \alpha \beta_{\mu \nu}-\stackrel{(12)}{z}_{\alpha \mu} \stackrel{(12)}{z}_{\nu} \alpha-\stackrel{(12)}{z}_{\alpha \nu} \stackrel{(12)}{z}_{\mu} \alpha_{-} \stackrel{(12)}{z}_{\mu \alpha} \stackrel{(12)}{z} \alpha_{\nu}-\stackrel{(12)}{z}_{\nu \alpha} \stackrel{(12)}{z}_{\mu}+ \\
& \left.+2 \stackrel{(11)}{z}_{\alpha \beta}+{ }^{-3}{ }^{\alpha \beta} g_{\mu \nu}-4 \stackrel{(11)}{z}_{\alpha \mu}+\bar{z}_{(\nu}^{-3} \alpha\right)-4 \stackrel{(11)}{z}_{\alpha \nu}+\stackrel{-3}{z}_{(\mu}^{\alpha)}+ \\
& +2 \stackrel{(22)}{z}_{\alpha \beta}+{ }^{-3}{ }^{\alpha \beta} g_{\mu \nu}-4 \stackrel{(22)}{z}_{\alpha \mu}+\bar{z}_{(\nu}^{-3}{ }^{\alpha)}-4 \stackrel{(22)}{z}_{\alpha \nu}+{ }^{z}{ }_{(\mu}^{\alpha)}+ \\
& +2 \stackrel{(13+)}{z}_{\alpha \beta}^{(13-)} \stackrel{(13 \beta}{z}_{g_{\mu \nu}-2} \stackrel{(13+)}{z}_{\alpha \mu} \stackrel{(13-)}{z}_{\nu}-2 \stackrel{(13+)}{z}_{\alpha \nu} \stackrel{(13-)}{z}_{\mu}-2 \stackrel{(13+)}{z}_{\mu \alpha}^{(13-)}{ }_{\nu}^{\alpha}-2 \stackrel{(13+)}{z}_{\nu \alpha}^{(13-)}{ }_{\mu}^{\alpha}+ \\
& +2 \stackrel{(23+)}{z}_{\alpha \beta}^{(23-)}{ }^{\alpha \beta} g_{\mu \nu}-2 \stackrel{(23+)}{z}_{\alpha \mu} \stackrel{(23-)}{z}{ }_{\nu}-2 \stackrel{(23+)}{z}_{\alpha \nu} \stackrel{(23-)}{z}_{\mu}-2 \stackrel{(23+)}{z}_{\mu \alpha}^{(23-)}{ }_{\nu}^{\alpha}-2 \stackrel{(23+)}{z}_{\nu \alpha}^{(23-)}{ }_{\mu}^{\alpha}+ \\
& +4 \stackrel{(13+)}{z}_{\alpha \beta} \stackrel{(23-)}{z}^{\alpha \beta} g_{\mu \nu}-4 \stackrel{(13+)}{z}_{\alpha \mu} \stackrel{(23-)}{z}_{\nu}-4 \stackrel{(13+)}{z}_{\alpha \nu} \stackrel{(23-)}{z}_{\mu}-4 \stackrel{(13+)}{z}_{\mu \alpha}^{(23-)}{ }_{\nu} \alpha-4 \stackrel{(13+)}{z}_{\nu \alpha}^{(23-)}{ }_{\mu}^{\alpha}+ \\
& +2 \stackrel{(12)}{z}_{\alpha \beta}+{ }_{z}^{-3} \alpha \beta g_{\mu \nu}-2 \stackrel{(12)}{z}_{\alpha \mu}+{ }_{z}^{-3}{ }_{\nu}-2 \stackrel{(12)}{z}_{\alpha \nu}+{ }_{z}^{-3}{ }_{\mu}-2 \stackrel{(12)}{z}_{\mu \alpha}+{ }_{z}^{-3}{ }_{\nu} \alpha-2 \stackrel{(12)}{z}_{\nu \alpha}+\bar{z}_{\mu}{ }^{\alpha}+
\end{aligned}
$$

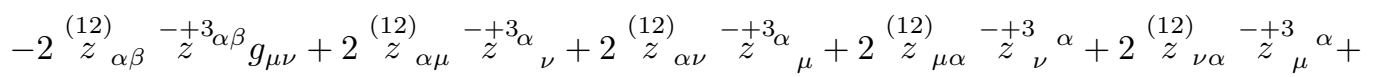


$+4 i \stackrel{(13+)}{z}_{\alpha \beta} \stackrel{(24-)}{\omega} \alpha \beta g_{\mu \nu}-4 i \stackrel{(13+)}{z}_{\alpha \mu} \stackrel{(24-)}{\omega} \alpha{ }_{\nu}-4 i \stackrel{(13+)}{z}_{\alpha \nu} \stackrel{(24-)}{\omega} \alpha{ }_{\mu}-4 i \stackrel{(13+)}{z}_{\mu \alpha} \stackrel{(24-)}{\omega}{ }_{\nu} \alpha+$ $-4 i \stackrel{(13+)}{z} \nu \alpha \stackrel{(24-)}{\omega}{ }_{\mu}^{\alpha}+$

$-4 i \stackrel{(13-)}{z}_{\alpha \beta} \stackrel{(24+)}{\omega} \alpha \beta g_{\mu \nu}+4 i \stackrel{(13-)}{z}_{\alpha \mu} \stackrel{(24+)}{\omega}_{\nu}+4 i \stackrel{(13-)}{z}_{\alpha \nu} \stackrel{(24+)}{\omega}_{\mu}+4 i \stackrel{(13-)}{z}_{\mu \alpha}^{(24+)}{ }_{\nu}^{\alpha}+$ $+4 i \stackrel{(13-)}{z}_{\nu \alpha}^{(24+)} \stackrel{(1)}{\omega}_{\mu}^{\alpha}+$

$+2 i \stackrel{(14-)}{\omega}_{\alpha \beta} \stackrel{(23+)}{z} \beta \alpha_{\beta} g_{\mu \nu}-2 i \stackrel{(23+)}{z} \alpha \mu \stackrel{(14-)}{\omega}_{\nu}^{\alpha}-2 i \stackrel{(23+)}{z}_{\alpha \nu} \stackrel{(14-)}{\omega}_{\mu} \alpha-2 i \stackrel{(23+)}{z} \mu \alpha \stackrel{(14-)}{\omega}{ }_{\nu}+$ $-2 i \stackrel{(23+)}{z}_{\nu \alpha}^{(14-)} \stackrel{(1)}{\omega}_{\mu}+$

$+2 i \stackrel{(14+)}{\omega}_{\alpha \beta} \stackrel{(23-)}{z} \beta \alpha_{\beta} g_{\mu \nu}-2 i \stackrel{(23-)}{z}_{\alpha \mu} \stackrel{(14+)}{\omega}_{\nu}^{\alpha}-2 i \stackrel{(23-)}{z}_{\alpha \nu} \stackrel{(14+)}{\omega}_{\mu} \alpha-2 i \stackrel{(23-)}{z}_{\mu \alpha}^{(14+)}{ }_{\alpha}{ }_{\nu}+$ $-2 i \stackrel{(23-)}{z} \nu \alpha \stackrel{(14+)}{\omega}{ }_{\mu}+$

$+8 i \stackrel{(24-)}{\omega}_{\alpha \beta} \stackrel{(13+)}{\omega} \beta \alpha g_{\mu \nu}-8 i \stackrel{(13+)}{\omega}_{\alpha \mu} \stackrel{(24-)}{\omega}{ }_{\nu} \alpha-8 i \stackrel{(13+)}{\omega}_{\alpha \nu} \stackrel{(24-)}{\omega}_{\mu} \alpha-8 i \stackrel{(13+)}{\omega}_{\mu \alpha} \stackrel{(24-)}{\omega}{ }_{\nu}+$ $-8 i \stackrel{(13+)}{\omega}_{\nu \alpha} \stackrel{(24-)}{\omega}{ }_{\mu}+$

$-8 i \stackrel{(24+)}{\omega}_{\alpha \beta} \stackrel{(13-)}{\omega}^{\prime} \alpha g_{\mu \nu}+8 i \stackrel{(13-)}{\omega}_{\alpha \mu} \stackrel{(24+)}{\omega}_{\nu} \alpha+8 i \stackrel{(13-)}{\omega}_{\alpha \nu} \stackrel{(24+)}{\omega}_{\mu} \alpha+8 i \stackrel{(13-)}{\omega}_{\mu \alpha}^{(24+)} \underset{\omega}{\omega}+$ $+8 i \stackrel{(13-)}{\omega}_{\nu \alpha} \stackrel{(24+)}{\omega}{ }_{\mu}+$

$+8 i \stackrel{(23+)}{\omega}_{\alpha \beta} \stackrel{(14-)}{\omega}^{\prime} \alpha g_{\mu \nu}-8 i \stackrel{(14-)}{\omega}_{\alpha \mu} \stackrel{(23+)}{\omega}{ }_{\nu} \alpha-8 i \stackrel{(14-)}{\omega}_{\alpha \nu} \stackrel{(23+)}{\omega}_{\mu} \alpha-8 i \stackrel{(14-)}{\omega}_{\mu \alpha}^{(23+)} \alpha{ }_{\nu}+$ $-8 i \stackrel{(14-)}{\omega}_{\nu \alpha} \stackrel{(23+)}{\omega}{ }_{\mu}+$

$-8 i \stackrel{(23-)}{\omega}_{\alpha \beta} \stackrel{(14+)}{\omega}_{\beta \alpha} g_{\mu \nu}+8 i \stackrel{(14+)}{\omega}_{\alpha \mu} \stackrel{(23-)}{\omega}{ }_{\nu} \alpha+8 i \stackrel{(14+)}{\omega}_{\alpha \nu} \stackrel{(23-)}{\omega}_{\mu} \alpha+8 i \stackrel{(14+)}{\omega}_{\mu \alpha}^{(23-)} \stackrel{(}{\omega}_{\nu}+$ $+8 i \stackrel{(14+)}{\omega}_{\nu \alpha} \stackrel{(23-)}{\omega}{ }_{\mu}+$

$+i \stackrel{(24+)}{z} \alpha \beta \stackrel{(13-)}{z} \beta \alpha g_{\mu \nu}-i \stackrel{(13-)}{z}_{\alpha \mu} \stackrel{(24+)}{z}_{\nu}^{\alpha}-i \stackrel{(13-)}{z}_{\alpha \nu} \stackrel{(24+)}{z}_{\mu}^{\alpha}-i \stackrel{(13-)}{z}_{\mu \alpha}^{(24+)}{ }_{\alpha}{ }_{\nu}+$ $-i \stackrel{(13-)}{z}_{\nu \alpha}^{(24+)_{\alpha}}{ }_{\mu}+$

$-i \stackrel{(24-)}{z} \alpha \beta \stackrel{(13+)}{z} \beta \alpha g_{\mu \nu}+i \stackrel{(13+)}{z}_{\alpha \mu} \stackrel{(24-)}{z}_{\nu}^{\alpha}+i \stackrel{(13+)}{z}_{\alpha \nu} \stackrel{(24-)}{z}_{\mu}^{\alpha}+i \stackrel{(13+)}{z}_{\mu \alpha} \stackrel{(24-)}{z}_{\nu}+$ $+i \stackrel{(13+)}{z}_{\nu \alpha}^{(24-)}{ }_{z}{ }_{\mu}+$

$+i \stackrel{(23-)}{z}_{\alpha \beta} \stackrel{(14+)}{z} \beta \alpha g_{\mu \nu}-i \stackrel{(14+)}{z}_{\alpha \mu} \stackrel{(23-)}{z}_{\nu}^{\alpha}-i \stackrel{(14+)}{z}_{\alpha \nu} \stackrel{(23-)}{z}_{\mu} \alpha-i \stackrel{(14+)}{z}_{\mu \alpha}^{(23-)}{ }_{z}{ }_{\nu}+$ $-i \stackrel{(14+)}{z}_{\nu \alpha}^{(23-)}{ }^{(23}{ }_{\mu}+$

$-i \stackrel{(23+)}{z} \alpha \beta \stackrel{(14-)}{z} \beta \alpha g_{\mu \nu}+i \stackrel{(14-)}{z}_{\alpha \mu} \stackrel{(23+)}{z}_{\nu}^{\alpha}+i \stackrel{(14-)}{z}_{\alpha \nu} \stackrel{(23+)}{z}_{\mu}^{\alpha}+i \stackrel{(14-)}{z}_{\mu \alpha}^{(23+)_{\alpha}}{ }_{\nu}+$ $+i \stackrel{(14-)}{z}_{\nu \alpha}^{(23+)}{ }^{(23}{ }_{\mu}+$ 
$+2 i \stackrel{(23+)}{\omega}_{\alpha \beta} \stackrel{(14-)}{z} \beta \alpha_{\beta} g_{\mu \nu}-2 i \stackrel{(14-)}{z}_{\alpha \mu} \stackrel{(23+)}{\omega}{ }_{\nu} \alpha-2 i \stackrel{(14-)}{z}_{\alpha \nu} \stackrel{(23+)}{\omega}_{\mu} \alpha-2 i \stackrel{(14-)}{z} \mu \alpha \stackrel{(23+)}{\omega} \alpha \underset{\nu}{ }+$ $-2 i \stackrel{(14-)}{z}_{\nu \alpha} \stackrel{(23+)}{\omega}{ }_{\mu}+$

$-2 i \stackrel{(23-)}{\omega}_{\alpha \beta} \stackrel{(14+)}{z} \beta \alpha_{\mu \nu}+2 i \stackrel{(14+)}{z}_{\alpha \mu} \stackrel{(23-)}{\omega}_{\nu}^{\alpha}+2 i \stackrel{(14+)}{z}_{\alpha \nu} \stackrel{(23-)}{\omega}_{\mu} \alpha+2 i \stackrel{(14+)}{z}_{\mu \alpha} \stackrel{(23-)}{\omega}{ }_{\nu}+$ $+2 i \stackrel{(14+)}{z} \nu \alpha \stackrel{(23-)}{\omega}{ }_{\mu}+$

$+2 i \stackrel{(13+)}{\omega}_{\alpha \beta} \stackrel{(24-)}{z} \beta \alpha_{\mu \nu}-2 i \stackrel{(24-)}{z}_{\alpha \mu} \stackrel{(13+)}{\omega}_{\nu} \alpha-2 i \stackrel{(24-)}{z}_{\alpha \nu} \stackrel{(13+)}{\omega}_{\mu} \alpha-2 i \stackrel{(24-)}{z} \mu \alpha \stackrel{(13+)}{\omega}{ }_{\nu}+$ $-2 i \stackrel{(24-)}{z}_{\nu \alpha}^{(13+)} \stackrel{(1)}{\omega}_{\mu}+$

$-2 i \stackrel{(13-)}{\omega}_{\alpha \beta} \stackrel{(24+)}{z} \beta \alpha_{\beta} g_{\mu \nu}+2 i \stackrel{(24+)}{z}_{\alpha \mu} \stackrel{(13-)}{\omega}_{\nu}^{\alpha}+2 i \stackrel{(24+)}{z}_{\alpha \nu} \stackrel{(13-)}{\omega}_{\mu} \alpha+2 i \stackrel{(24+)}{z} \mu \alpha \stackrel{(13-)}{\omega}{ }_{\nu}+$ $+2 i \stackrel{(24+)}{z}_{\nu \alpha} \stackrel{(13-)}{\omega}{ }_{\mu}+$

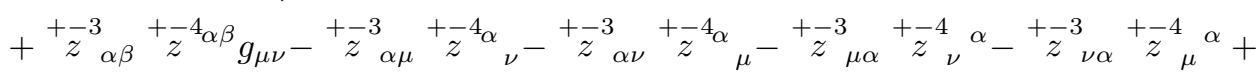

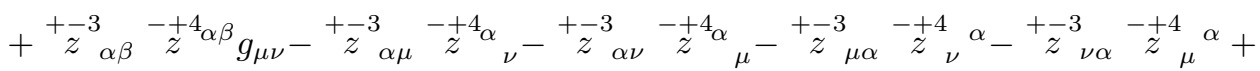

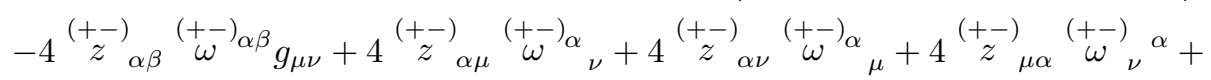

$+4 \stackrel{(+-)}{z}_{\nu \alpha} \stackrel{(+-)}{\omega}{ }_{\mu} \alpha+$

$+4 \stackrel{(+-)}{z}_{\alpha \beta} \stackrel{(-+)}{\omega}^{\alpha \beta} g_{\mu \nu}-4 \stackrel{(+-)}{z}_{\alpha \mu} \stackrel{(-+)}{\omega}_{\alpha}{ }_{\nu}-4 \stackrel{(+-)}{z}_{\alpha \nu} \stackrel{(-+)}{\omega}_{\mu}-4 \stackrel{(+-)}{z}_{\mu \alpha} \stackrel{(-+)}{\omega}_{\nu} \alpha+$

$-4 \stackrel{(+-)}{z}_{\nu \alpha} \stackrel{(-+)}{\omega}{ }_{\mu} \alpha+$

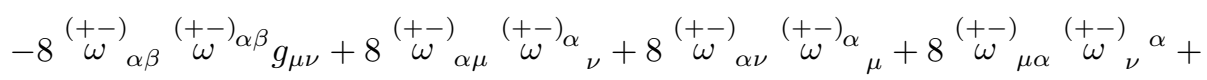

$+8 \stackrel{(+-)}{\omega}_{\nu \alpha} \stackrel{(+-}{\omega}_{\mu} \alpha+$

$+8 \stackrel{(+-)}{\omega}_{\alpha \beta} \stackrel{(-+)}{\omega}_{\alpha \beta} g_{\mu \nu}-8 \stackrel{(+-)}{\omega}_{\alpha \mu} \stackrel{(-+)}{\omega}_{\nu}-8 \stackrel{(++)}{\omega}_{\alpha \nu} \stackrel{(-+)}{\omega}_{\mu}-8 \stackrel{(+-)}{\omega}_{\mu \alpha} \stackrel{(-+)}{\omega}_{\nu}{ }+$ $-8 \stackrel{(+-)}{\omega}_{\nu \alpha} \stackrel{(-+)}{\omega}{ }_{\mu}^{\alpha}+$ 


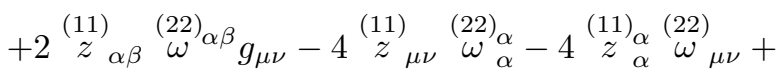

$$
\begin{aligned}
& +4 \stackrel{(11)}{\omega}_{\alpha \beta} \stackrel{(22)}{\omega}_{\alpha \beta} g_{\mu \nu}-8 \stackrel{(11)}{\omega}_{\mu \nu} \stackrel{(22)}{\omega}_{\alpha}-8 \stackrel{(11)}{\omega}_{\alpha} \stackrel{(22)}{\omega}_{\mu \nu}+
\end{aligned}
$$

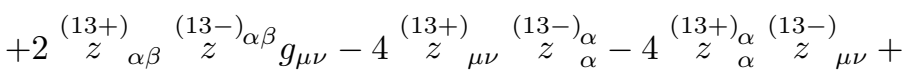

$$
\begin{aligned}
& +2 \stackrel{(23+)}{z}{ }_{\alpha \beta} \stackrel{(23-)}{z} \alpha \beta g_{\mu \nu}-4 \stackrel{(23+)}{z}_{\mu \nu}^{(23--)} \underset{\alpha}{z}-4{\underset{z}{z}}_{\alpha}^{(23+)_{\alpha}} z_{\mu \nu}^{(23-)}+
\end{aligned}
$$

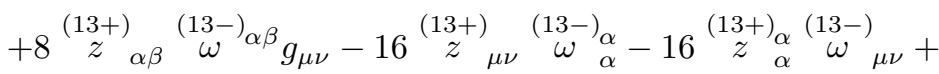

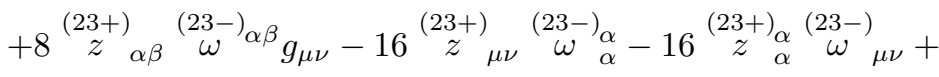

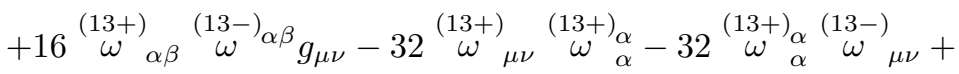

$$
\begin{aligned}
& +4\left(\stackrel{(11)}{z}_{\alpha \beta}+\stackrel{(22)}{z}_{\alpha \beta}+2 \stackrel{(11)}{\omega}_{\alpha \beta}+2 \stackrel{(22)}{\omega}_{\alpha \beta}+\stackrel{(+-3)}{\omega}_{\alpha \beta}+2 \stackrel{(12)}{z}_{\alpha \beta}+4 \stackrel{(12)}{\omega}_{\alpha \beta}\right)+{ }^{+3}{ }^{3}{ }_{\alpha \beta} g_{\mu \nu}+ \\
& -4\left(\stackrel{(11)}{z}_{\mu \nu}+\stackrel{(22)}{z}_{\mu \nu}+2 \stackrel{(11)}{\omega}_{\mu \nu}+2 \stackrel{(22)}{\omega}_{\mu \nu}+\stackrel{(+-3)}{\omega}_{\mu \nu}+2 \stackrel{(12)}{z}_{\mu \nu}+4 \stackrel{(12)}{\omega}_{\mu \nu}\right){ }^{+-3}{ }_{\alpha}+ \\
& -4\left(\stackrel{(11)}{z}_{\alpha}^{\alpha}+\stackrel{(22)}{z}_{\alpha} \alpha+2 \stackrel{(11)}{\omega}_{\alpha}^{\alpha}+2 \stackrel{(22)}{\omega}_{\alpha}^{\alpha}+\stackrel{(+-3)}{\omega}_{\alpha} \alpha+2 \stackrel{(12)}{z}_{\alpha}^{\alpha}+4 \stackrel{(12)}{\omega}_{\alpha} \alpha\right)+\overline{-}^{+3}{ }_{\mu \nu}+
\end{aligned}
$$

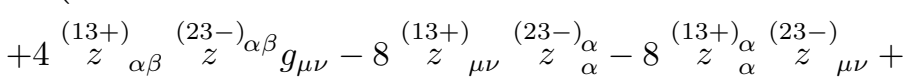

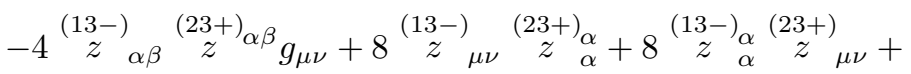

$$
\begin{aligned}
& +8 \stackrel{(13+)}{z}_{\alpha \beta} \stackrel{(13-)}{\omega}{ }^{\alpha \beta} g_{\mu \nu}-16 \stackrel{(13+)}{z}_{\mu \nu} \stackrel{(13-)}{\omega}_{\alpha}-16 \stackrel{(13+)}{z}_{\alpha} \stackrel{(13-)}{\omega}_{\mu \nu}+
\end{aligned}
$$

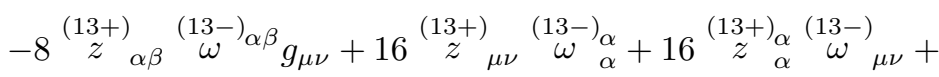

$$
\begin{aligned}
& +16 \stackrel{(13+)}{\omega}_{\alpha \beta} \stackrel{(23-)}{\omega}^{\alpha \beta} g_{\mu \nu}-32 \stackrel{(13+)}{\omega}_{\mu \nu} \stackrel{(23-)}{\omega}_{\alpha}-32 \stackrel{(13+)}{\omega}_{\alpha} \stackrel{(23-)}{\omega}_{\mu \nu}+
\end{aligned}
$$

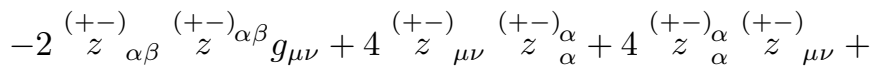

$$
\begin{aligned}
& +4 \stackrel{(+-)}{z}_{\alpha \beta} \stackrel{(+-)}{\omega}_{\alpha \beta} g_{\mu \nu}+4 \stackrel{(+-)}{z}_{\mu \nu} \stackrel{(+-)}{\omega}_{\alpha}+4 \stackrel{(+-}{z}_{\alpha} \stackrel{(++)}{\omega}_{\mu \nu}+ \\
& +8 \stackrel{(+-)}{\omega}_{\alpha \beta} \stackrel{(+-)}{\omega}_{\alpha \beta} g_{\mu \nu}+16 \stackrel{(+-)}{\omega}_{\mu \nu} \stackrel{(+-)}{\omega}_{\alpha}+16 \stackrel{(+-)}{\omega}_{\alpha} \stackrel{(+-)}{\omega}_{\mu \nu}+
\end{aligned}
$$

The above relationships satisfy the expected relationship

$$
\Theta_{\mu \nu}=\Theta_{\nu \mu}
$$

For spliting the spin-1 and spin-0 sectors the energy momentum tensor can also be rewritten in terms of transverse and longitudinal pieces [22].

\section{Vectorial Energy-Momentum Tensor}

The physical content for Faraday invisible lines is to rewrite the energy-momentum conservation law (5.1) in its vectorial expression. It yields the following conservation laws 


$$
\begin{aligned}
& \frac{\partial U}{\partial t}+\vec{\nabla} \cdot \vec{S}=0 \\
& \frac{\partial S}{\partial t}+\vec{\nabla} \cdot \overleftrightarrow{T}=0
\end{aligned}
$$

Eqs.(6.1) are differentials describing the EM flow through energy density, Poynting Vector and Stress tensor. We should write down the fields strengths in vectorial form. Defining

$$
\begin{aligned}
& F_{[\mu \nu]}=\left(\begin{array}{cccc}
0 & E_{x} & E_{y} & E_{z} \\
-E_{x} & 0 & -B_{z} & B_{y} \\
-E_{y} & B_{z} & 0 & -B_{x} \\
-E_{z} & -B_{y} & B_{x} & 0
\end{array}\right), z_{[\mu \nu]}=\left(\begin{array}{cccc}
0 & e_{x} & e_{y} & e_{z} \\
-e_{x} & 0 & -b_{z} & b_{y} \\
-e_{y} & b_{z} & 0 & -b_{x} \\
-e_{z} & -b_{y} & b_{x} & 0
\end{array}\right), \\
& S_{\mu \nu}=\left(\begin{array}{cccc}
S & S_{x} & S_{y} & S_{z} \\
S_{x} & S_{x x} & \stackrel{\leftrightarrow}{S}_{z} & \stackrel{S}{S}_{y} \\
S_{y} & \overleftrightarrow{S}_{z} & S_{y y} & \overleftrightarrow{S}_{x} \\
S_{z} & \overleftrightarrow{S}_{y} & \overleftrightarrow{S}_{x} & S_{z z}
\end{array}\right), z_{(\mu \nu)}=\left(\begin{array}{cccc}
s & s_{x} & s_{y} & s_{z} \\
s_{x} & s_{x x} & \overleftrightarrow{s}_{z} & \overleftrightarrow{s}_{y} \\
s_{y} & \overleftrightarrow{s}_{z} & s_{y y} & \overleftrightarrow{s}_{x} \\
s_{z} & \overleftrightarrow{s}_{y} & \overleftrightarrow{s}_{x} & s_{z z}
\end{array}\right)
\end{aligned}
$$

we have

$$
\begin{aligned}
& F_{0 i} \equiv \vec{E}_{A i}, \quad F_{i j} \equiv \epsilon_{i j k} \vec{B}_{A k}, \quad U_{0 i} \equiv \vec{E}_{U i}, \quad U_{i j} \equiv \epsilon_{i j k} \vec{B}_{U k}, \quad V_{0 i}^{ \pm} \equiv \vec{E}_{ \pm i}, \quad V_{i j}^{ \pm} \equiv \epsilon_{i j k} \vec{B}_{ \pm k}, \\
& S_{00}^{1} \equiv S_{A}, \quad S_{0 i}^{1} \equiv \vec{S}_{A}, \quad S_{i j}^{1} \equiv \stackrel{\leftrightarrow}{S}_{A}, S_{00}^{2} \equiv S_{U}, \quad S_{0 i}^{2} \equiv \vec{S}_{U}, \quad S_{i j}^{2} \equiv \stackrel{\leftrightarrow}{S}_{U}, \quad S_{00}^{ \pm} \equiv S_{ \pm i}, \\
& S_{0 i}^{ \pm} \equiv \vec{S}_{ \pm}, \quad S_{i j}^{ \pm} \equiv \stackrel{\leftrightarrow}{S}_{ \pm}, \quad z_{[0 i]} \equiv \vec{e}_{i}, \quad z_{[i j]} \equiv \epsilon_{i j k} \vec{b}_{k}, \quad z_{(00)} \equiv s, \quad z_{(0 i)} \equiv \vec{s}, \quad z_{(i j)} \equiv \stackrel{\leftrightarrow}{s} \\
& \omega_{[0 i]} \equiv \vec{e}_{\omega i}, \quad \omega_{[i j]} \equiv \epsilon_{i j k} \vec{b}_{\omega k}, \quad \omega_{(00)} \equiv s_{\omega}, \quad \omega_{(0 i)} \equiv \vec{s}_{\omega}, \quad \omega_{(i j)} \equiv \stackrel{\leftrightarrow}{s}_{\omega}
\end{aligned}
$$

\section{Energy Density in Vectorial Form}

The energy density of the system is

$$
U=U_{K}+U_{M}+U_{G F}+U_{I}
$$

Working out (5.2), one gets for $\mathcal{L}_{K}^{A}$

$$
U_{K}^{A}=a_{1}\left(\left|\vec{E}_{A}\right|^{2}+\left|\vec{B}_{A}\right|^{2}\right)+a_{2}\left(\left|\vec{E}_{U}\right|^{2}+\left|\vec{B}_{U}\right|^{2}\right)+a_{3}\left(\vec{E}_{+} \cdot \vec{E}_{-}+\vec{B}_{+} \cdot \vec{B}_{-}\right)
$$

which is positively well defined. 
For $\mathcal{L}_{K}^{S}$ :

$$
\begin{aligned}
& U_{K}^{S}=-\frac{3}{2} b_{(11)} S_{A}^{2}-b_{11} \vec{S}_{A} \cdot \vec{S}_{A}+\frac{1}{2} \stackrel{\leftrightarrow}{S}_{A} \cdot \stackrel{\leftrightarrow}{S}_{A}+ \\
& -\frac{3}{2} b_{(22)} S_{U}^{2}-b_{(22)} \vec{S}_{U} \cdot \vec{S}_{U}+\frac{1}{2} b_{(22)} \stackrel{\leftrightarrow}{S}_{U} \cdot \stackrel{\leftrightarrow}{S}_{U}-3 b_{(33)} S_{+} S_{-}-2 b_{(33)} \vec{S}_{+} \cdot \vec{S}_{-}+b_{33} \stackrel{\leftrightarrow}{S}_{+} \cdot \stackrel{\leftrightarrow}{S}_{-} \\
& -\frac{5}{2} c_{(11)} S_{A}^{2}-3 c_{(11)} S_{A} S_{i A}^{i}-\frac{1}{2} c_{(11)} S_{i A}^{i 2}-\frac{5}{2} c_{(22)} S_{U}^{2}-3 c_{(22)} S_{U} S_{i U}^{i}-\frac{1}{2} c_{(22)} S_{i U}^{i 2}+ \\
& -3 c_{(33)} S_{+} S_{-}-4 c_{(33)} S_{+} S_{-i}^{i}-4 c_{(33)} S_{-} S_{+i}^{i}+c_{(33)} S_{i+}^{i} S_{j-}^{j}
\end{aligned}
$$

which is not well defined positively. Its positive analysis is shown at Appendix B. Consequently, it yields that eqs.(7.2) and eq.(7.3) are showing a health ground state. Saying that, the model is stable. Limited by below.

For $\mathcal{L}_{M}$ :

$$
U_{M}=\frac{3}{4} m_{U}^{2} \phi_{U}^{2}-\frac{1}{4} m_{u}^{2}|\vec{U}|^{2}+\frac{3}{2} m_{ \pm}^{2} \phi_{+} \phi_{-}-\frac{1}{2} m_{ \pm}^{2} \vec{V}_{+} \cdot \vec{V}_{-}
$$

For $\mathcal{L}_{G F}$ :

$$
\begin{aligned}
& U_{\mathrm{GF}}=-\frac{5}{8} \xi_{(11)} S_{A}^{2}-\frac{3}{4} \xi_{(11)} S_{A} S_{A i}^{i}-\frac{1}{8} \xi_{(11)} S_{A i}^{2 i}-\frac{5}{8} \xi_{(22)} S_{U}^{2}-\frac{3}{4} \xi_{(22)} S_{U} S_{U i}^{i}-\frac{1}{8} \xi_{(22)} S_{U i}^{i 2}+ \\
& -\frac{3}{2} \xi_{(12)} S_{A} S_{U}-2 \xi_{(12)} S_{A} S_{U i}^{i}-2 \xi_{(12)} S_{U} S_{A i}^{i}+\frac{1}{2} \xi_{(12)} S_{A i}^{i} S_{U j}^{j}+ \\
& -\frac{3}{2} \xi_{(33)} S_{+} S_{-}-2 \xi_{(33)} S_{+} S_{-i}^{i}-2 \xi_{(33)} S_{-} S_{+i}^{i}+\frac{1}{2} \xi_{(33)} S_{+i}^{i} S_{-j}^{j}
\end{aligned}
$$

For $\mathcal{L}_{I}^{3 A}$ :

$$
\begin{aligned}
& U_{I}^{3 A}=4 b_{1}\left(\vec{E}_{A} \cdot \vec{e}^{[12]}+\vec{B}_{A} \cdot \vec{b}^{[12]}\right)+4 b_{2}\left(\vec{E}_{U} \cdot \vec{e}^{[12]}+\vec{B}_{U} \cdot \vec{b}^{[12]}\right)+ \\
& +4 b_{1}\left(\vec{E}_{A} \cdot \vec{e}^{[+-]}+\vec{B}_{U} \cdot \vec{b}^{[+-]}\right)+4 b_{2}\left(\vec{E}_{U} \cdot \vec{e}^{[+-]}+\vec{B}_{U} \cdot \vec{b}^{[+-]}\right)+ \\
& +4 \beta_{1}\left(\vec{E}_{A} \cdot \vec{e}^{(+-)}+\vec{B}_{U} \cdot \vec{b}^{(+-)}\right)+4 \beta_{2}\left(\vec{E}_{U} \cdot \vec{e}^{(+-)}+\vec{B}_{U} \cdot \vec{b}^{(+-)}\right)+ \\
& +4 b_{3}\left(\vec{E}_{+} \cdot \vec{e}^{[-1]}+\vec{B}_{+} \cdot \vec{b}^{[-1]}+\vec{E}_{+} \cdot \vec{e}^{[-2]}+\vec{B}_{+} \cdot \vec{b}^{[-2]}\right)+ \\
& +4 b_{3}\left(\vec{E}_{-} \cdot \vec{e}^{[+1]}+\vec{B}_{-} \cdot \vec{b}^{[+1]}+\vec{E}_{-} \cdot \vec{e}^{[+2]}+\vec{B}_{-} \cdot \vec{b}^{[+2]}\right)
\end{aligned}
$$

For $\mathcal{L}_{I}^{3 S}$ 


$$
\begin{aligned}
& U_{I}^{3 S}=-3 \beta_{1} S_{1} s^{(11)}-2 \beta_{1} \vec{S}_{1} \cdot \vec{s}^{(11)}+\beta_{1} \stackrel{\leftrightarrow}{S}_{1} \cdot \stackrel{\leftrightarrow}{s}(11)+ \\
& -3 \beta_{1} S_{1} s^{(22)}-2 \beta_{1} \vec{S}_{1} \cdot \vec{s}^{(22)}+\beta_{1} \stackrel{\leftrightarrow}{S}_{1} \cdot \stackrel{\leftrightarrow}{s}^{(22)}+ \\
& -3 \beta_{1} S_{1} s^{(12)}-2 \beta_{1} \vec{S}_{1} \cdot \vec{s}^{(12)}+\beta_{1} \stackrel{\leftrightarrow}{S}_{1} \cdot \stackrel{\leftrightarrow}{s}^{(12)}+ \\
& -3 \beta_{1} S_{1} s^{+-3}-2 \beta_{1} \vec{S}_{1} \cdot \vec{s}^{+-3}+\beta_{1} \stackrel{\leftrightarrow}{S}_{1} \cdot \stackrel{\leftrightarrow}{s}^{+-3}+ \\
& -3 \beta_{2} S_{2} s^{(11)}-2 \beta_{2} \vec{S}_{2} \cdot \vec{s}^{(11)}+\beta_{2} \stackrel{\leftrightarrow}{S}_{2} \cdot \stackrel{\leftrightarrow}{s}(11)+ \\
& -3 \beta_{2} S_{2} s^{(22)}-2 \beta_{2} \vec{S}_{2} \cdot \vec{s}^{(22)}+\beta_{2} \stackrel{\leftrightarrow}{S}_{2} \cdot \stackrel{\leftrightarrow}{s}^{(22)}+ \\
& -3 \beta_{2} S_{2} s^{(12)}-2 \beta_{2} \vec{S}_{2} \cdot \vec{s}^{(12)}+\beta_{2} \stackrel{\leftrightarrow}{S}_{2} \cdot \stackrel{\leftrightarrow}{s}^{(12)}+ \\
& -3 \beta_{2} S_{2} s^{+-3}-2 \beta_{2} \vec{S}_{2} \cdot \vec{s}^{+-3}+\beta_{2} \stackrel{\leftrightarrow}{S}_{2} \cdot \stackrel{\leftrightarrow}{s}{ }^{+-3}+ \\
& -6 \beta_{3} S_{+} s^{(-1)}-4 \beta_{3} \vec{S}_{+} \cdot \vec{s}^{(-1)}+2 \beta_{3} \overleftrightarrow{S}_{+} \cdot \stackrel{\leftrightarrow}{s}(-1)+ \\
& -6 \beta_{3} S_{+} s^{(-2)}-4 \beta_{3} \vec{S}_{+} \cdot \vec{s}^{(-2)}+2 \beta_{3} \stackrel{\leftrightarrow}{S}_{+} \cdot \stackrel{\leftrightarrow}{s}^{(-2)}+ \\
& -6 \beta_{3} S_{-} s^{(+1)}-4 \beta_{3} \vec{S}_{-} \cdot \vec{s}^{(+1)}+2 \beta_{3} \stackrel{\leftrightarrow}{S}_{-} \cdot \stackrel{\leftrightarrow}{s}(+1)+ \\
& -6 \beta_{3} S_{-} s^{(+2)}-4 \beta_{3} \vec{S}_{-} \cdot \vec{s}^{(+2)}+2 \beta_{3} \stackrel{\leftrightarrow}{S}_{-} \cdot \stackrel{\leftrightarrow}{s}(+2)+ \\
& -3 \rho_{1} S_{1} s^{(11)}-4 \rho_{1} S_{1} s_{i}^{(11) i}-4 \rho_{1} s^{(11)} S_{1 i}^{i}+\rho_{1} S_{1 i}^{i} s_{j}^{(11) j} \\
& -3 \rho_{1} S_{1} s^{(22)}-4 \rho_{1} S_{1} s_{i}^{(22) i}-4 \rho_{1} s^{(22)} S_{1 i}^{i}+\rho_{1} S_{1 i}^{i} s_{j}^{(22) j} \\
& -3 \rho_{1} S_{1} s^{(12)}-4 \rho_{1} S_{1} s_{i}^{(12) i}-4 \rho_{1} s^{(12)} S_{1 i}^{i}+\rho_{1} S_{1 i}^{i} s_{j}^{(12) j} \\
& -3 \rho_{1} S_{1} s^{+-3}-4 \rho_{1} S_{1} s_{i}^{+-3 i}-4 \rho_{1} s^{+-3} S_{1 i}^{i}+\rho_{1} S_{1 i}^{i} s_{j}^{+-3 j} \\
& -3\left(\rho_{1}+4 \beta_{1}\right) S_{1} s_{\omega}^{(11)}-4\left(\rho_{1}+4 \beta_{1}\right) S_{1} s_{\omega i}^{(11) i}-4\left(\rho_{1}+4 \beta_{1}\right) s_{\omega}^{(11)} S_{1 i}^{i}+\left(\rho_{1}+4 \beta_{1}\right) S_{1 i}^{i} s_{\omega j}^{(11) j} \\
& -3\left(\rho_{1}+4 \beta_{1}\right) S_{1} s_{\omega}^{(22)}-4\left(\rho_{1}+4 \beta_{1}\right) S_{1} s_{\omega i}^{(22) i}-4\left(\rho_{1}+4 \beta_{1}\right) s_{\omega}^{(22)} S_{1 i}^{i}+\left(\rho_{1}+4 \beta_{1}\right) S_{1 i}^{i} s_{\omega j}^{(22) j} \\
& -6\left(\rho_{1}+4 \beta_{1}\right) S_{1} s_{\omega}^{(12)}-8\left(\rho_{1}+4 \beta_{1}\right) S_{1} s_{\omega i}^{(12) i}-8\left(\rho_{1}+4 \beta_{1}\right) s_{\omega}^{(12)} S_{1 i}^{i}+2\left(\rho_{1}+4 \beta_{1}\right) S_{1 i}^{i} s_{\omega j}^{(12) j} \\
& -6\left(\rho_{1}+4 \beta_{1}\right) S_{1} s_{\omega}^{+-3}-8\left(\rho_{1}+4 \beta_{1}\right) S_{1} s_{\omega i}^{+-3 i}-8\left(\rho_{1}+4 \beta_{1}\right) s_{\omega}^{+-3} S_{1 i}^{i}+2\left(\rho_{1}+4 \beta_{1}\right) S_{1 i}^{i} s_{\omega j}^{+-3 j} \\
& -3 \rho_{2} S_{2} s^{(11)}-4 \rho_{2} S_{2} s_{i}^{(11) i}-4 \rho_{2} s^{(11)} S_{2 i}^{i}+\rho_{2} S_{2 i}^{i} s_{j}^{(11) j} \\
& -3 \rho_{2} S_{2} s^{(22)}-4 \rho_{2} S_{2} s_{i}^{(22) i}-4 \rho_{1} s^{(22)} S_{1 i}^{i}+\rho_{2} S_{2 i}^{i} s_{j}^{(22) j} \\
& -3 \rho_{2} S_{2} s^{(12)}-4 \rho_{2} S_{2} s_{i}^{(12) i}-4 \rho_{1} s^{(12)} S_{1 i}^{i}+\rho_{2} S_{2 i}^{i} s_{j}^{(12) j} \\
& -3 \rho_{2} S_{2} s^{+-3}-4 \rho_{2} S_{2} s_{i}^{+-3 i}-4 \rho_{2} s^{+-3} S_{2 i}^{i}+\rho_{2} S_{2 i}^{i} s_{j}^{+-3 j} \\
& -3\left(\rho_{2}+4 \beta_{2}\right) S_{2} s_{\omega}^{(11)}-4\left(\rho_{2}+4 \beta_{2}\right) S_{1} s_{\omega i}^{(11) i}-4\left(\rho_{2}+4 \beta_{2}\right) s_{\omega}^{(11)} S_{2 i}^{i}+\left(\rho_{2}+4 \beta_{2}\right) S_{2 i}^{i} s_{\omega j}^{(11) j} \\
& -3\left(\rho_{2}+4 \beta_{2}\right) S_{2} s_{\omega}^{(22)}-4\left(\rho_{2}+4 \beta_{2}\right) S_{2} s_{\omega i}^{(22) i}-4\left(\rho_{2}+4 \beta_{2}\right) s_{\omega}^{(22)} S_{2 i}^{i}+\left(\rho_{2}+4 \beta_{2}\right) S_{2 i}^{i} s_{\omega j}^{(22) j}
\end{aligned}
$$




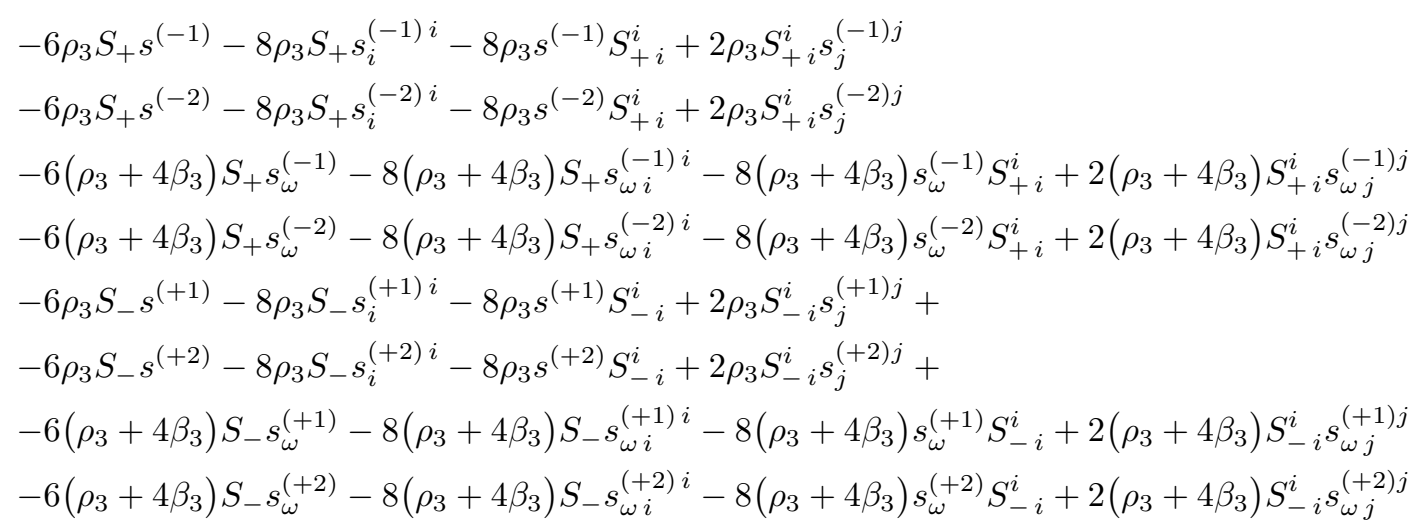

For $\mathcal{L}_{I}^{4 A}$ :

$$
\begin{aligned}
& U_{I}^{4 A}=2\left(\left|\vec{e}^{[12]}\right|^{2}+\left|\vec{b}^{[12]}\right|^{2}\right)+2\left(\vec{e}^{[12]} \cdot \vec{e}^{[21]}+\vec{b}^{[12]} \cdot \vec{b}^{[21]}\right)+ \\
& +4\left(\vec{e}^{[13+]} \cdot \vec{e}^{[13-]}+\vec{b}^{[13+]} \cdot \vec{b}^{[13-]}\right)+4\left(\vec{e}^{[23+]} \cdot \vec{e}^{[23-]}+\vec{b}^{[23+]} \cdot \vec{b}^{[23-]}\right)+ \\
& +8\left(\vec{e}^{[13+]} \cdot \vec{e}^{[23-]}+\vec{b}^{[13+]} \cdot \vec{b}^{[23-]}\right)+4\left(\vec{e}^{[12]} \cdot \vec{e}^{[+-]}+\vec{b}^{[12]} \cdot \vec{b}^{[+-]}\right) \\
& -8\left(\vec{e}^{(12)} \cdot \vec{e}_{\omega}^{(12)}+\vec{b}^{(12)} \cdot \vec{b}_{\omega}^{(12)}\right)-16\left(\left|\vec{e}_{\omega}^{(12)}\right|^{2}+\left|\vec{b}_{\omega}^{(12)}\right|^{2}\right)+ \\
& +4\left(\vec{e}^{(13+)} \cdot \vec{e}^{(13-)}+\vec{b}^{(13+)} \cdot \vec{b}^{(13-)}\right)+4\left(\vec{e}^{(23+)} \cdot \vec{e}^{(23-)}+\vec{b}^{(23+)} \cdot \vec{b}^{(23-)}\right)+ \\
& +8\left(\vec{e}^{(13+)} \cdot \vec{e}^{(23-)}+\vec{b}^{(13+)} \cdot \vec{b}^{(23-)}\right)-4 i\left(\vec{e}^{(13+)} \cdot \vec{e}_{\omega}^{(24-)}+\vec{b}^{(13+)} \cdot \vec{b}_{\omega}^{(24-)}\right)+ \\
& +4 i\left(\vec{e}^{(13-)} \cdot \vec{e}_{\omega}^{(24+)}+\vec{b}^{(13-)} \cdot \vec{b}_{\omega}^{(24+)}\right)-4 i\left(\vec{e}^{(23+)} \cdot \vec{e}_{\omega}^{(14-)}+\vec{b}^{(23+)} \cdot \vec{b}_{\omega}^{(14-)}\right)+ \\
& +4 i\left(\vec{e}^{(23-)} \cdot \vec{e}_{\omega}^{(14+)}+\vec{b}^{(23-)} \cdot \vec{b}_{\omega}^{(14+)}\right)-16 i\left(\vec{e}_{\omega}^{(13+)} \cdot \vec{e}_{\omega}^{(24-)}+\vec{b}_{\omega}^{(13+)} \cdot \vec{b}_{\omega}^{(24-)}\right)+ \\
& +16 i\left(\vec{e}_{\omega}^{(13-)} \cdot \vec{e}_{\omega}^{(24+)}+\vec{b}_{\omega}^{(13-)} \cdot \vec{b}_{\omega}^{(24+)}\right)-16 i\left(\vec{e}_{\omega}^{(14-)} \cdot \vec{e}_{\omega}^{(23+)}+\vec{b}_{\omega}^{(14-)} \cdot \vec{b}_{\omega}^{(23+)}\right)+ \\
& +16 i\left(\vec{e}_{\omega}^{(14+)} \cdot \vec{e}_{\omega}^{(23-)}+\vec{b}_{\omega}^{(14+)} \cdot \vec{b}_{\omega}^{(23-)}\right)-2 i\left(\vec{e}^{(13-)} \cdot \vec{e}^{(24+)}+\vec{b}^{(13-)} \cdot \vec{b}^{(24+)}\right)+ \\
& +2 i\left(\vec{e}^{(13+)} \cdot \vec{e}^{(24-)}+\vec{b}^{(13+)} \cdot \vec{b}^{(24-)}\right)-2 i\left(\vec{e}^{(14+)} \cdot \vec{e}^{(23-)}+\vec{b}^{(14+)} \cdot \vec{b}^{(23-)}\right)+ \\
& +2 i\left(\vec{e}^{(14-)} \cdot \vec{e}^{(23+)}+\vec{b}^{(14-)} \cdot \vec{b}^{(23+)}\right)-4 i\left(\vec{e}^{(14-)} \cdot \vec{e}_{\omega}^{(23+)}+\vec{b}^{(14-)} \cdot \vec{b}_{\omega}^{(23+)}\right)+ \\
& +4 i\left(\vec{e}^{(14+)} \cdot \vec{e}_{\omega}^{(23-)}+\vec{b}^{(14+)} \cdot \vec{b}_{\omega}^{(23-)}\right)-4 i\left(\vec{e}^{(24-)} \cdot \vec{e}_{\omega}^{(13+)}+\vec{b}^{(24-)} \cdot \vec{b}_{\omega}^{(13+)}\right) \\
& +4 i\left(\vec{e}^{(24+)} \cdot \vec{e}_{\omega}^{(13-)}+\vec{b}^{(24+)} \cdot \vec{b}_{\omega}^{(13-)}\right)-16\left(\vec{e}^{(+-)} \cdot \vec{e}_{\omega}^{(+-)}+\vec{b}^{(+-)} \cdot \vec{b}_{\omega}^{(+-)}\right)+ \\
& -32\left(\vec{e}_{\omega}^{(+-)} \cdot \vec{e}_{\omega}^{(++)}+\vec{b}_{\omega}^{(+-)} \cdot \vec{b}_{\omega}^{(++)}\right)
\end{aligned}
$$

For $\mathcal{L}_{I}^{4 S}$ : 


$$
\begin{aligned}
& U_{I}^{4 S}=-3 s^{[12] 2}-2\left|\vec{s}^{[12]}\right|^{2}+\left|\stackrel{\leftrightarrow}{s}^{[12]}\right|^{2}-3 s^{[12]} s^{[21]}-2 \vec{s}^{[12]} \cdot \vec{s}^{[21]}+\stackrel{\leftrightarrow}{s}^{[12]} \cdot \stackrel{\leftrightarrow}{s}^{[21]}+
\end{aligned}
$$

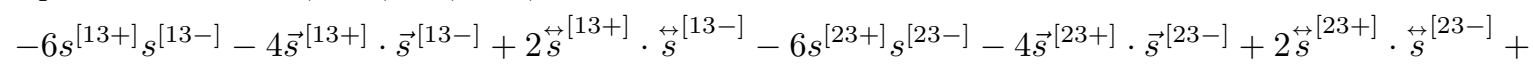

$$
\begin{aligned}
& -12 s^{[13+]} s^{[23-]}-8 \vec{s}^{[13+]} \cdot \vec{s}^{[23-]}+4 \overleftrightarrow{s}^{[13+]} \cdot \overleftrightarrow{s}^{[23-]}-6\left|s^{[+-]}\right|^{2}-4\left|\vec{s}^{[+-]}\right|^{2}+2\left|\overleftrightarrow{s}^{[+-]}\right|^{2}+
\end{aligned}
$$

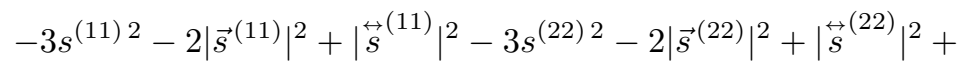

$$
\begin{aligned}
& -6 s^{(11)} s_{\omega}^{(11)}-4 \vec{s}^{(11)} \cdot \vec{s}_{\omega}^{(11)}+2 \stackrel{\leftrightarrow}{s}^{(11)} \cdot \stackrel{\leftrightarrow}{s}_{\omega}^{(11)}-6 s^{(11)} s_{\omega}^{(22)}-4 \vec{s}^{(22)} \cdot \vec{s}_{\omega}^{(22)}+2 \stackrel{\leftrightarrow}{s}^{(22)} \cdot \stackrel{\leftrightarrow}{s}_{\omega}^{(22)}+ \\
& -6 s_{\omega}^{(11) 2}-4\left|\vec{s}_{\omega}^{(11)}\right|^{2}+2\left|\stackrel{\leftrightarrow}{s}_{\omega}^{(11)}\right|^{2}-6 s_{\omega}^{(22) 2}-4\left|\vec{s}_{\omega}^{(22)}\right|^{2}+2\left|\stackrel{\leftrightarrow}{s}_{\omega}^{(22)}\right|^{2}+
\end{aligned}
$$

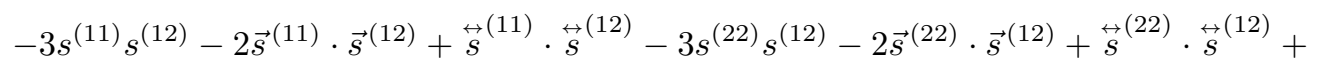

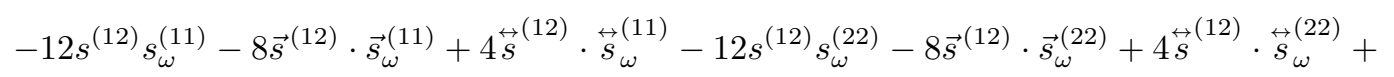

$$
\begin{aligned}
& -24 s_{\omega}^{(11)} s_{\omega}^{(12)}-16 \vec{s}_{\omega}^{(11)} \cdot \vec{s}_{\omega}^{(12)}+8 \stackrel{\leftrightarrow}{s}_{\omega}^{(11)} \cdot \stackrel{\leftrightarrow}{s}_{\omega}^{(12)}-24 s_{\omega}^{(22)} s_{\omega}^{(12)}-16 \vec{s}_{\omega}^{(22)} \cdot \vec{s}_{\omega}^{(12)}+8 \stackrel{\leftrightarrow}{s}_{\omega}^{(22)} \cdot \stackrel{\leftrightarrow}{s}_{\omega}^{(12)}+ \\
& -3 s^{(11)} s^{(22)}-2 \vec{s}^{(11)} \cdot \vec{s}^{(22)}+\overleftrightarrow{s}^{(11)} \cdot \overleftrightarrow{s}^{(22)}-6 s^{(11)} s^{+-3}-4 \vec{s}^{(11)} \cdot \vec{s}^{+-3}+2 \overleftrightarrow{s}^{(11)} \cdot \overleftrightarrow{s}^{+-3}+ \\
& -6 s^{(22)} s^{+-3}-4 \vec{s}^{(22)} \cdot \vec{s}^{+-3}+2 \stackrel{\leftrightarrow}{s}^{(22)} \cdot \stackrel{\leftrightarrow}{s}^{+-3}-6 s^{(12) 2}-4\left|\vec{s}^{(12)}\right|^{2}+2\left|\overleftrightarrow{s}^{(12)}\right|^{2}+ \\
& -12 s^{(12)} s_{\omega}^{(12)}-8 \vec{s}^{(12)} \cdot \vec{s}_{\omega}^{(12)}+4 \overleftrightarrow{s}^{(12)} \cdot \stackrel{\leftrightarrow}{s}_{\omega}^{(12)}-24 s_{\omega}^{(12) 2}-16\left|\vec{s}_{\omega}^{(12)}\right|^{2}+8\left|\stackrel{\leftrightarrow}{s}_{\omega}^{(12)}\right|^{2}+
\end{aligned}
$$

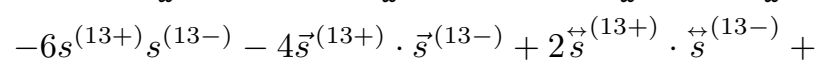

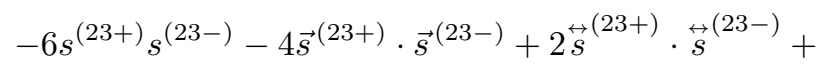

$$
\begin{aligned}
& -12 s^{(13+)} s^{(23-)}-8 \vec{s}^{(13+)} \cdot \vec{s}^{(23-)}+4 \stackrel{\leftrightarrow}{s}^{(13+)} \cdot \stackrel{\leftrightarrow}{s}^{(23-)}+ \\
& -12 s^{(12)} s^{+-3}-8 \vec{s}^{(12)} \cdot \vec{s}^{+-3}+4 \overleftrightarrow{s}^{(12)} \cdot \overleftrightarrow{s}^{+-3}+ \\
& -6 s^{+-3} s^{+-4}-4 \vec{s}^{+-3} \cdot \vec{s}^{+-4}+2 \overleftrightarrow{s}^{+-3} \cdot \stackrel{\leftrightarrow}{s}{ }^{+-4}+ \\
& -6 i s^{(13+)} s_{\omega}^{(24-)}-4 i \vec{s}^{(13+)} \cdot \vec{s}_{\omega}^{(24-)}+2 i \stackrel{\leftrightarrow}{s}(13+) \cdot \stackrel{\leftrightarrow}{s}_{\omega}^{(24-)}+ \\
& +6 i s^{(13-)} s_{\omega}^{(24+)}+4 i \vec{s}^{(13-)} \cdot \vec{s}_{\omega}^{(24+)}-2 i \stackrel{\leftrightarrow}{s}(13-) \cdot \stackrel{\leftrightarrow}{s}_{\omega}^{(24+)}+ \\
& -6 i s^{(23+)} s_{\omega}^{(14-)}-4 i \vec{s}^{(23+)} \cdot \vec{s}_{\omega}^{(14-)}+2 i \stackrel{\leftrightarrow}{s}(23+) \cdot \stackrel{\leftrightarrow}{s}_{\omega}^{(14-)}+ \\
& +6 i s^{(23-)} s_{\omega}^{(14+)}+4 i \vec{s}^{(23-)} \cdot \vec{s}_{\omega}^{(14+)}-2 i \stackrel{\leftrightarrow}{s}(23-) \cdot \stackrel{\leftrightarrow}{s}_{\omega}^{(14+)}+ \\
& -24 i s_{\omega}^{(13+)} s_{\omega}^{(24-)}-16 i \vec{s}_{\omega}^{(13+)} \cdot \vec{s}_{\omega}^{(24-)}+8 i \stackrel{\leftrightarrow}{s}_{\omega}^{(13+)} \cdot \stackrel{\leftrightarrow}{s}_{\omega}^{(24-)}+ \\
& +24 i s_{\omega}^{(13-)} s_{\omega}^{(24+)}+16 i \vec{s}_{\omega}^{(13-)} \cdot \vec{s}_{\omega}^{(24+)}-8 i \stackrel{\leftrightarrow}{s}_{\omega}^{(13-)} \cdot \overleftrightarrow{s}_{\omega}^{(24+)}+
\end{aligned}
$$

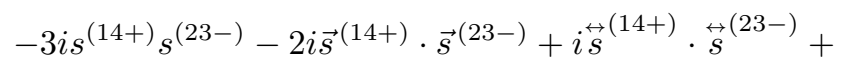

$$
\begin{aligned}
& -3 i s^{(14-)} s_{\omega}^{(23+)}-2 i \vec{s}^{(14-)} \cdot \vec{s}_{\omega}^{(23+)}+i \stackrel{\leftrightarrow}{s}^{(14-)} \cdot \stackrel{\leftrightarrow}{s}^{(23+)}+ \\
& +24 i s_{\omega}^{(14+)} s_{\omega}^{(23-)}+16 i \vec{s}_{\omega}^{(14+)} \cdot \vec{s}_{\omega}^{(23-)}-8 i \stackrel{\leftrightarrow}{s}_{\omega}^{(14+)} \cdot \stackrel{\leftrightarrow}{s}_{\omega}^{(23-)}+ \\
& -24 i s_{\omega}^{(14-)} s_{\omega}^{(23+)}-16 i \vec{s}_{\omega}^{(14-)} \cdot \vec{s}_{\omega}^{(23+)}+8 i \stackrel{\leftrightarrow}{s}_{\omega}^{(14-)} \cdot \stackrel{\leftrightarrow}{s}_{\omega}^{(23+)}+
\end{aligned}
$$

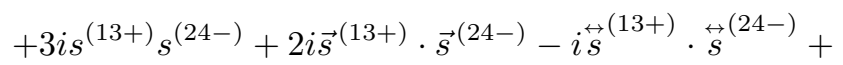

$$
\begin{aligned}
& -3 i s^{(13-)} s^{(24+)}-2 i \vec{s}^{(13-)} \cdot \vec{s}^{(24+)}+i \stackrel{\leftrightarrow}{s}^{(13-)} \cdot \stackrel{\leftrightarrow}{s}^{(24+)}+
\end{aligned}
$$




$$
\begin{aligned}
& +6 i s^{(14+)} s_{\omega}^{(23-)}+4 i \vec{s}^{(14+)} \cdot \vec{s}_{\omega}^{(23-)}-2 i \stackrel{\leftrightarrow}{s}(14+) \cdot \stackrel{\leftrightarrow}{s}_{\omega}^{(23-)}+ \\
& -6 i s^{(14-)} s_{\omega}^{(23+)}-4 i \vec{s}^{(14-)} \cdot \vec{s}_{\omega}^{(23+)}+2 i \stackrel{\leftrightarrow}{s}^{(14-)} \cdot \stackrel{\leftrightarrow}{s}_{\omega}^{(23+)}+ \\
& +6 i s^{(24+)} s_{\omega}^{(13-)}+4 i \vec{s}^{(24+)} \cdot \vec{s}_{\omega}^{(13-)}-2 i \stackrel{\leftrightarrow}{s}(24+) \cdot \stackrel{\leftrightarrow}{s}_{\omega}^{(13-)}+ \\
& -6 i s^{(24-)} s_{\omega}^{(13+)}-4 i \vec{s}^{(24-)} \cdot \vec{s}_{\omega}^{(13+)}+2 i \stackrel{\leftrightarrow}{s}(24-) \cdot \stackrel{\leftrightarrow}{s}_{\omega}^{(13+)}+ \\
& +10 s^{[13+]} s^{[13-]}+6 s^{[13+]} s_{i}^{[13-] i}+6 s^{[13-]} s_{i}^{[13+] i}-2 s_{i}^{[13+] i} s_{j}^{[13-] j}+ \\
& +10 s^{[23+]} s^{[23-]}+6 s^{[23+]} s_{i}^{[23-] i}+6 s^{[23-]} s_{i}^{[23+] i}-2 s_{i}^{[23+] i} s_{j}^{[23-] j}+ \\
& +10 s^{[+-] 2}+12 s^{[+-]} s_{i}^{[+-] i}-2\left(s_{i}^{[+-] i}\right)^{2}+ \\
& +10 s^{[13+]} s^{[23-]}+6 s^{[13+]} s_{i}^{[23-] i}+6 s^{[23-]} s_{i}^{[13+] i}-2 s_{i}^{[13+] i} s_{j}^{[23-] j}+ \\
& -10 s^{[13-]} s^{[23+]}-6 s^{[13-]} s_{i}^{[23+] i}-6 s^{[23+]} s_{i}^{[13-] i}+2 s_{i}^{[13-] i} s_{j}^{[23+] j}+ \\
& +10 s^{[13+]} s^{[24-]}+6 s^{[13+]} s_{i}^{[24-] i}+6 s^{[24-]} s_{i}^{[13+] i}-2 s_{i}^{[13+] i} s_{j}^{[24-] j}+ \\
& -10 s^{[13-]} s^{[24+]}-6 s^{[13-]} s_{i}^{[24+] i}-6 s^{[24+]} s_{i}^{[13-] i}+2 s_{i}^{[13-] i} s_{j}^{[24+] j}+ \\
& -10 s^{(11)} s_{\omega}^{(22)}-6 s^{(11)} s_{\omega i}^{(22) i}-6 s_{\omega}^{(22)} s_{i}^{(11) i}+2 s_{i}^{(11) i} s_{\omega j}^{(22) j}+ \\
& -20 s_{\omega}^{(11)} s_{\omega}^{(22)}-12 s_{\omega}^{(11)} s_{\omega i}^{(22) i}-12 s_{\omega}^{(22)} s_{\omega i}^{(11) i}+4 s_{\omega i}^{(11) i} s_{\omega j}^{(22) j}+ \\
& -10 s^{(13+)} s^{(13-)}-6 s^{(13+)} s_{i}^{(13-) i}-6 s^{(13-)} s_{i}^{(13+) i}+2 s_{i}^{(13-) i} s_{j}^{(13+) j}+ \\
& -10 s^{(23+)} s^{(23-)}-6 s^{(23+)} s_{i}^{(23-) i}-6 s^{(23-)} s_{i}^{(23+) i}+2 s_{i}^{(23-) i} s_{j}^{(23+) j}+ \\
& -40 s^{(13+)} s_{\omega}^{(13-)}-24 s^{(13+)} s_{\omega i}^{(13-) i}-24 s_{\omega}^{(13-)} s_{i}^{(13+) i}+8 s_{\omega i}^{(13-) i} s_{j}^{(13+) j}+ \\
& -40 s^{(23+)} s_{\omega}^{(23-)}-24 s^{(23+)} s_{\omega i}^{(23-) i}-24 s_{\omega}^{(23-)} s_{i}^{(23+) i}+8 s_{\omega i}^{(23-) i} s_{j}^{(23+) j}+ \\
& -80 s_{\omega}^{(13+)} s_{\omega}^{(13-)}-48 s_{\omega}^{(13+)} s_{\omega i}^{(13-) i}-48 s_{\omega}^{(13-)} s_{\omega i}^{(13+) i}+16 s_{\omega i}^{(13-) i} s_{\omega j}^{(13+) j}+ \\
& -80 s_{\omega}^{(23+)} s_{\omega}^{(23-)}-48 s_{\omega}^{(23+)} s_{\omega i}^{(23-) i}-48 s_{\omega}^{(23-)} s_{\omega i}^{(23+) i}+16 s_{\omega i}^{(23-) i} s_{\omega j}^{(23+) j}+ \\
& -10 s^{(13+)} s^{(23-)}-6 s^{(13+)} s_{i}^{(23-) i}-6 s^{(23-)} s_{i}^{(13+) i}+2 s_{i}^{(23-) i} s_{j}^{(13+) j}+ \\
& +10 s^{(13-)} s^{(23+)}+6 s^{(13-)} s_{i}^{(23+) i}+6 s^{(23+)} s_{i}^{(13-) i}-2 s_{i}^{(23+) i} s_{j}^{(13-) j}+ \\
& -40 s^{(13+)} s_{\omega}^{(23-)}-24 s^{(13+)} s_{\omega i}^{(23-) i}-24 s_{\omega}^{(23-)} s_{i}^{(13+) i}+8 s_{\omega i}^{(23-) i} s_{j}^{(13+) j}+ \\
& +40 s^{(13-)} s_{\omega}^{(23+)}+24 s^{(13-)} s_{\omega i}^{(23+) i}+24 s_{\omega}^{(23+)} s_{i}^{(13-) i}-8 s_{\omega i}^{(23+) i} s_{j}^{(13-) j}+ \\
& -80 s_{\omega}^{(13+)} s_{\omega}^{(23-)}-48 s_{\omega}^{(13+)} s_{\omega i}^{(23-) i}-48 s_{\omega}^{(23-)} s_{\omega i}^{(13+) i}+16 s_{\omega i}^{(23-) i} s_{\omega j}^{(13+) j}+ \\
& +80 s_{\omega}^{(13-)} s_{\omega}^{(23+)}+48 s_{\omega}^{(13-)} s_{\omega i}^{(23+) i}+48 s_{\omega}^{(23+)} s_{\omega i}^{(13-) i}-16 s_{\omega i}^{(23+) i} s_{\omega j}^{(13-) j}+ \\
& +10 s_{\omega}^{(+-)} s_{\omega}^{(+-)}+12 s_{\omega}^{(+-)} s_{\omega i}^{(+-) i}-2 s_{\omega i}^{(+-) i} s_{\omega j}^{(+-) j}+ \\
& -20 s^{+-3} s_{\omega}^{+-4}-12 s^{+-3} s_{\omega i}^{+-4 i}-12 s_{\omega}^{+-4} s_{i}^{+-3 i}+4 s_{i}^{+-3 i} s_{\omega j}^{+-4 j}+ \\
& -40 s_{\omega}^{+-3} s_{\omega}^{+-4}-24 s_{\omega}^{+-3} s_{\omega i}^{+-4 i}-24 s_{\omega}^{+-4} s_{\omega i}^{+-3 i}+8 s_{\omega i}^{+-3 i} s_{\omega j}^{+-4 j}+ \\
& -20 s^{(11)} s_{\omega}^{+-3}-12 s^{(11)} s_{\omega i}^{+-3 i}-12 s_{\omega}^{+-3} s_{i}^{(11) i}+4 s_{i}^{(11) i} s_{\omega j}^{+-3 j}+ \\
& -20 s^{(22)} s_{\omega}^{+-3}-12 s^{(22)} s_{\omega i}^{+-3 i}-12 s_{\omega}^{+-3} s_{i}^{(22) i}+4 s_{i}^{(22) i} s_{\omega j}^{+-3 j}+ \\
& -40 s_{\omega}^{(11)} s_{\omega}^{+-3}-24 s_{\omega}^{(11)} s_{\omega i}^{+-3 i}-24 s_{\omega}^{+-3} s_{\omega i}^{(11) i}+8 s_{\omega i}^{(11) i} s_{\omega j}^{+-3 j}+ \\
& -40 s_{\omega}^{(22)} s_{\omega}^{+-3}-24 s_{\omega}^{(22)} s_{\omega i}^{+-3 i}-24 s_{\omega}^{+-3} s_{\omega i}^{(22) i}+8 s_{\omega i}^{(22) i} s_{\omega j}^{+-3 j}+ \\
& -40 s^{(12)} s_{\omega}^{+-3}-24 s^{(12)} s_{\omega i}^{+-3 i}-24 s_{\omega}^{+-3} s_{i}^{(12) i}+8 s_{i}^{(12) i} s_{\omega j}^{+-3 j}+ \\
& -80 s_{\omega}^{(12)} s_{\omega}^{+-3}-48 s_{\omega}^{(12)} s_{\omega i}^{+-3 i}-48 s_{\omega}^{+-3} s_{\omega i}^{(12) i}+16 s_{\omega i}^{(12) i} s_{\omega j}^{+-3 j}+
\end{aligned}
$$


Notice that above equations are showing the energies for particles with spin-1 and spin- 0 .

\section{Poynting Vector in Vectorial Form}

The total flux density of the system is

$$
\vec{S}=\vec{S}_{K}+\vec{S}_{M}+\vec{S}_{G F}+\vec{S}_{I}
$$

For $\mathcal{L}_{K}^{A}$ :

$$
\vec{S}_{K}^{A}=-2 a_{1} \vec{E}_{A} \times \vec{B}_{A}-2 a_{2} \vec{E}_{U} \times \vec{B}_{U}-4 a_{3} \vec{E}_{+} \times \vec{B}_{-}
$$

For $\mathcal{L}_{K}^{S}$ :

$$
\begin{aligned}
& \vec{S}_{K}^{S}=-2 b_{(11)} S_{A} \vec{S}_{A}-2 b_{(11)} \vec{S}_{A} \cdot \stackrel{\leftrightarrow}{S}_{A}-2 b_{(22)} S_{U} \vec{S}_{U}-2 b_{(22)} \vec{S}_{U} \cdot \stackrel{\leftrightarrow}{S}_{U} \\
& -2 b_{(33)} S_{+} \vec{S}_{-}-2 b_{(33)} S_{-} \vec{S}_{+}-2 b_{(11)} \vec{S}_{+} \cdot \stackrel{\leftrightarrow}{S}_{-}-2 b_{(11)} \vec{S}_{-} \cdot \stackrel{\leftrightarrow}{S}_{+} \\
& -2 c_{(11)} \vec{S}_{A} S_{A}-2 c_{(11)} \vec{S}_{A} S_{i A}^{i}-2 c_{(22)} \vec{S}_{U} S_{U}-2 c_{(22)} \vec{S}_{U} S_{i U}^{i}+ \\
& -2 c_{(33)} \vec{S}_{+} S_{-}-2 c_{(33)} \vec{S}_{-} S_{+}-2 c_{(33)} \vec{S}_{+} S_{i-}^{i}-2 c_{(33)} \vec{S}_{-} S_{i+}^{i}
\end{aligned}
$$

For $\mathcal{L}_{M}$ :

$$
\vec{S}_{M}=\frac{1}{2} m_{U}^{2} \phi \vec{U}+\frac{1}{2} m_{ \pm}^{2} \phi_{+} \vec{V}_{-}+\frac{1}{2} m_{ \pm}^{2} \phi_{-} \vec{V}_{+}
$$

For $\mathcal{L}_{G F}$ :

$$
\begin{aligned}
& \vec{S}_{G F}=-\frac{1}{2} \xi_{(11)} \vec{S}_{A} S_{A}-\frac{1}{2} \xi_{(11)} \vec{S}_{A} S_{A i}^{i}-\frac{1}{2} \xi_{(22)} \vec{S}_{U} S_{U}-\frac{1}{2} \xi_{(22)} \vec{S}_{U} S_{U i}^{i}+ \\
& -\xi_{(12)} \vec{S}_{A} S_{U}-\xi_{(12)} \vec{S}_{U} S_{A}-\xi_{(33)} \vec{S}_{A} S_{U i}^{i}-\xi_{(33)} \vec{S}_{U} S_{A i}^{i} \\
& -\xi_{(33)} \vec{S}_{+} S_{-}-\xi_{(33)} \vec{S}_{-} S_{+}-\xi_{(33)} \vec{S}_{+} S_{-i}^{i}-\xi_{(33)} \vec{S}_{-} S_{+i}^{i}
\end{aligned}
$$

For $\mathcal{L}_{I}^{3 A}$ : 


$$
\begin{aligned}
& \vec{S}_{I}^{3 \mathrm{~A}}=-4 b_{1}\left(\vec{E}_{A} \times \vec{b}^{[12]}+\vec{B}_{A} \times \vec{e}^{[12]}\right)-4 b_{2}\left(\vec{E}_{U} \times \vec{b}^{[12]}+\vec{B}_{U} \times \vec{e}^{[12]}\right)+ \\
& -4 b_{1}\left(\vec{E}_{A} \times \vec{b}^{[+-]}+\vec{B}_{U} \times \vec{e}^{[+-]}\right)-4 b_{2}\left(\vec{E}_{U} \times \vec{b}^{[+-]}+\vec{B}_{U} \times \vec{e}^{[+-]}\right)+ \\
& -4 \beta_{1}\left(\vec{E}_{A} \times \vec{b}^{(+-)}+\vec{B}_{U} \times \vec{e}^{(+-)}\right)-4 \beta_{2}\left(\vec{E}_{U} \times \vec{b}^{(+-)}+\vec{B}_{U} \times \vec{e}^{(+-)}\right)+ \\
& -4 b_{3}\left(\vec{E}_{+} \times \vec{b}^{[-1]}+\vec{B}_{+} \times \vec{e}^{[-1]}+\vec{E}_{+} \times \vec{b}^{[-2]}+\vec{B}_{+} \times \vec{e}^{[-2]}\right)+ \\
& -4 b_{3}\left(\vec{E}_{-} \times \vec{b}^{[+1]}+\vec{B}_{-} \times \vec{e}^{[+1]}+\vec{E}_{-} \times \vec{b}^{[+2]}+\vec{B}_{-} \times \vec{e}^{[+2]}\right)
\end{aligned}
$$

Notice that eq.(8.6) introduces new terms for Poynting vectors beyond that ones derived from constitutive Maxwell equations [23]. 
For $\mathcal{L}_{I}^{3 \mathrm{~S}}$ :

$$
\begin{aligned}
& \vec{S}_{I}^{3 \mathrm{~S}}=-2 \beta_{1} S_{1} \vec{s}^{(11)}-2 \beta_{1} s^{(11)} \vec{S}_{1}-2 \beta_{1} \vec{S}_{1} \cdot \stackrel{\leftrightarrow}{s}^{(11)}-2 \beta_{1} \vec{s}^{(11)} \cdot \stackrel{\leftrightarrow}{S}_{1} \\
& -2 \beta_{1} S_{1} \vec{s}^{(22)}-2 \beta_{1} s^{(22)} \vec{S}_{1}-2 \beta_{1} \vec{S}_{1} \cdot \stackrel{\leftrightarrow}{s}^{(22)}-2 \beta_{1} \vec{s}^{(22)} \cdot \stackrel{\leftrightarrow}{S}_{1} \\
& -4 \beta_{1} S_{1} \vec{s}^{(12)}-4 \beta_{1} s^{(12)} \vec{S}_{1}-4 \beta_{1} \vec{S}_{1} \cdot \stackrel{\leftrightarrow}{s}{ }^{(12)}-4 \beta_{1} \vec{s}^{(12)} \cdot \stackrel{\leftrightarrow}{S}_{1} \\
& -4 \beta_{1} S_{1} \vec{s}^{+-3}-4 \beta_{1} s^{+-3} \vec{S}_{1}-4 \beta_{1} \vec{S}_{1} \cdot \stackrel{\leftrightarrow}{s}{ }^{+-3}-4 \beta_{1} \vec{s}^{+-3} \cdot \stackrel{\leftrightarrow}{S}_{1} \\
& -2 \beta_{2} S_{2} \vec{s}^{(11)}-2 \beta_{2} s^{(11)} \vec{S}_{2}-2 \beta_{2} \vec{S}_{2} \cdot \stackrel{\leftrightarrow}{s}(11)-2 \beta_{2} \vec{s}^{(11)} \cdot \stackrel{\leftrightarrow}{S}_{2} \\
& -2 \beta_{2} S_{2} \vec{s}^{(22)}-2 \beta_{2} s^{(22)} \vec{S}_{2}-2 \beta_{2} \vec{S}_{2} \cdot \stackrel{\leftrightarrow}{s}{ }^{(22)}-2 \beta_{2} \vec{s}^{(22)} \cdot \stackrel{\leftrightarrow}{S}_{2} \\
& -4 \beta_{2} S_{2} \vec{s}^{(12)}-4 \beta_{2} s^{(12)} \vec{S}_{2}-4 \beta_{2} \vec{S}_{2} \cdot \stackrel{\leftrightarrow}{s}{ }^{(12)}-4 \beta_{2} \vec{s}^{(12)} \cdot \stackrel{\leftrightarrow}{S}_{2} \\
& -4 \beta_{2} S_{2} \vec{s}^{+-3}-4 \beta_{2} s^{+-3} \vec{S}_{2}-4 \beta_{2} \vec{S}_{2} \cdot \stackrel{\leftrightarrow}{s}^{+-3}-4 \beta_{2} \vec{s}^{+-3} \cdot \stackrel{\leftrightarrow}{S}_{2} \\
& -4 \beta_{3} S_{+} \vec{s}^{(-1)}-4 \beta_{3} s^{(-1)} \vec{S}_{+}-4 \beta_{3} \vec{S}_{+} \cdot \stackrel{\leftrightarrow}{s}{ }^{(-1)}-4 \beta_{3} \vec{s}^{(-1)} \cdot \stackrel{\leftrightarrow}{S}_{+} \\
& -4 \beta_{3} S_{+} \vec{s}^{(-2)}-4 \beta_{3} s^{(-2)} \vec{S}_{+}-4 \beta_{3} \vec{S}_{+} \cdot \stackrel{\leftrightarrow}{s}(-2)-4 \beta_{3} \vec{s}^{(-2)} \cdot \stackrel{\leftrightarrow}{S}_{+} \\
& -4 \beta_{3} S_{-} \vec{s}^{(+1)}-4 \beta_{3} s^{(+1)} \vec{S}_{-}-4 \beta_{3} \vec{S}_{-} \cdot \stackrel{\leftrightarrow}{s}(+1)-4 \beta_{3} \vec{s}^{(+1)} \cdot \stackrel{\leftrightarrow}{S}_{-} \\
& -4 \beta_{3} S_{-} \vec{s}^{(+2)}-4 \beta_{3} s^{(+2)} \vec{S}_{-}-4 \beta_{3} \vec{S}_{-} \cdot \stackrel{\leftrightarrow}{s}^{(+2)}-4 \beta_{3} \vec{s}^{(+2)} \cdot \overleftrightarrow{S}_{-} \\
& -2 \rho_{1} \vec{S}_{1} s^{(11)}-2 \rho_{1} S_{1} \vec{s}^{(11)}-2 \rho_{1} \vec{S}_{1} s_{i}^{(11) i}-2 \rho_{1} \vec{s}^{(11)} S_{1 i}^{i} \\
& -2 \rho_{1} \vec{S}_{1} s^{(22)}-2 \rho_{1} S_{1} \vec{s}^{(22)}-2 \rho_{1} \vec{S}_{1} s_{i}^{(22) i}-2 \rho_{1} \vec{s}^{(22)} S_{1 i}^{i} \\
& -4 \rho_{1} \vec{S}_{1} s^{(12)}-4 \rho_{1} S_{1} \vec{s}^{(12)}-4 \rho_{1} \vec{S}_{1} s_{i}^{(12) i}-4 \rho_{1} \vec{s}^{(12)} S_{1 i}^{i} \\
& -4 \rho_{1} \vec{S}_{1} s^{+-3}-4 \rho_{1} S_{1} \vec{s}^{+-3}-4 \rho_{1} \vec{S}_{1} s_{i}^{+-3 i}-4 \rho_{1} \vec{s}^{+-3} S_{1 i}^{i} \\
& -2\left(\rho_{1}+4 \beta_{1}\right) \vec{S}_{1} s_{\omega}^{(11)}-2\left(\rho_{1}+4 \beta_{1}\right) S_{1} \vec{s}_{\omega}^{(11)}-2\left(\rho_{1}+4 \beta_{1}\right) \vec{S}_{1} s_{\omega i}^{(11) i}-2\left(\rho_{1}+4 \beta_{1}\right) \vec{s}_{\omega}^{(11)} S_{1 i}^{i} \\
& -2\left(\rho_{1}+4 \beta_{1}\right) \vec{S}_{1} s_{\omega}^{(22)}-2\left(\rho_{1}+4 \beta_{1}\right) S_{1} \vec{s}_{\omega}^{(22)}-2\left(\rho_{1}+4 \beta_{1}\right) \vec{S}_{1} s_{\omega i}^{(22) i}-2\left(\rho_{1}+4 \beta_{1}\right) \vec{s}_{\omega}^{(22)} S_{1 i}^{i} \\
& -4\left(\rho_{1}+4 \beta_{1}\right) \vec{S}_{1} s_{\omega}^{(12)}-4\left(\rho_{1}+4 \beta_{1}\right) S_{1} \vec{s}_{\omega}^{(12)}-4\left(\rho_{1}+4 \beta_{1}\right) \vec{S}_{1} s_{\omega i}^{(12) i}-4\left(\rho_{1}+4 \beta_{1}\right) \vec{s}^{(12)} S_{1 i}^{i} \\
& -4\left(\rho_{1}+4 \beta_{1}\right) \vec{S}_{1} s_{\omega}^{+-3}-4\left(\rho_{1}+4 \beta_{1}\right) S_{1} \vec{s}_{\omega}^{+-3}-4\left(\rho_{1}+4 \beta_{1}\right) \vec{S}_{1} s_{i}^{+-3 i}-4\left(\rho_{1}+4 \beta_{1}\right) \vec{s}^{+-3} S_{1 i}^{i} \\
& -2 \rho_{2} \vec{S}_{2} s^{(11)}-2 \rho_{2} S_{2} \vec{s}^{(11)}-2 \rho_{2} \vec{S}_{2} s_{i}^{(11) i}-2 \rho_{2} \vec{s}^{(11)} S_{2 i}^{i} \\
& -2 \rho_{1} \vec{S}_{2} s^{(22)}-2 \rho_{2} S_{2} \vec{s}^{(22)}-2 \rho_{2} \vec{S}_{2} s_{i}^{(22) i}-2 \rho_{2} \vec{s}^{(22)} S_{2 i}^{i} \\
& -4 \rho_{1} \vec{S}_{2} s^{(12)}-4 \rho_{2} S_{2} \vec{s}^{(12)}-4 \rho_{2} \vec{S}_{2} s_{i}^{(12) i}-4 \rho_{2} \vec{s}^{(12)} S_{2 i}^{i} \\
& -4 \rho_{1} \vec{S}_{2} s^{+-3}-4 \rho_{2} S_{2} \vec{s}^{+-3}-4 \rho_{2} \vec{S}_{2} s_{i}^{+-3 i}-4 \rho_{2} \vec{s}^{+-3} S_{2 i}^{i} \\
& -2\left(\rho_{2}+4 \beta_{2}\right) \vec{S}_{2} s_{\omega}^{(11)}-2\left(\rho_{2}+4 \beta_{2}\right) S_{2} \vec{s}_{\omega}^{(11)}-2\left(\rho_{2}+4 \beta_{2}\right) \vec{S}_{2} s_{\omega i}^{(11) i}-2\left(\rho_{2}+4 \beta_{2}\right) \vec{s}_{\omega}^{(11)} S_{1 i}^{i} \\
& -2\left(\rho_{2}+4 \beta_{2}\right) \vec{S}_{2} s_{\omega}^{(22)}-2\left(\rho_{2}+4 \beta_{2}\right) S_{2} \vec{s}_{\omega}^{(22)}-2\left(\rho_{2}+4 \beta_{2}\right) \vec{S}_{2} s_{\omega i}^{(22) i}-2\left(\rho_{2}+4 \beta_{2}\right) \vec{s}_{\omega}^{(22)} S_{2 i}^{i} \\
& -4\left(\rho_{2}+4 \beta_{2}\right) \vec{S}_{2} s_{\omega}^{(12)}-4\left(\rho_{2}+4 \beta_{2}\right) S_{2} \vec{S}_{\omega}^{(12)}-4\left(\rho_{2}+4 \beta_{2}\right) \vec{S}_{2} s_{\omega i}^{(12) i}-4\left(\rho_{2}+4 \beta_{2}\right) \vec{s}^{(12)} S_{2 i}^{i} \\
& -4\left(\rho_{2}+4 \beta_{2}\right) \vec{S}_{2} s_{\omega}^{+-3}-4\left(\rho_{2}+4 \beta_{2}\right) S_{2} \vec{s}_{\omega}^{+-3}-4\left(\rho_{2}+4 \beta_{2}\right) \vec{S}_{2} s_{i}^{+-3 i}-4\left(\rho_{2}+4 \beta_{2}\right) \vec{s}^{+-3} S_{2 i}^{i}
\end{aligned}
$$




$$
\begin{aligned}
& -4 \rho_{3} \vec{S}_{+} s^{(-1)}-2 \rho_{3} S_{+} \vec{s}^{(-1)}-2 \rho_{3} \vec{S}_{+} s_{i}^{(-1) i}-2 \rho_{3} \vec{s}^{(-1)} S_{+i}^{i} \\
& -4 \rho_{3} \vec{S}_{+} s^{(-2)}-2 \rho_{3} S_{+} \vec{s}^{(-2)}-2 \rho_{3} \vec{S}_{+} s_{i}^{(-2) i}-2 \rho_{3} \vec{s}^{(-2)} S_{+i}^{i} \\
& -4\left(\rho_{3}+4 \beta_{3}\right) \vec{S}_{+} s_{\omega}^{(-1)}-2\left(\rho_{3}+4 \beta_{3}\right) S_{+} \vec{s}_{\omega}^{(-1)}-2\left(\rho_{3}+4 \beta_{3}\right) \vec{S}_{+} s_{\omega i}^{(-1) i}-2\left(\rho_{3}+4 \beta_{3}\right) \vec{s}_{\omega}^{(-1)} S_{+i}^{i} \\
& -4\left(\rho_{3}+4 \beta_{3}\right) \vec{S}_{+} s_{\omega}^{(-2)}-2\left(\rho_{3}+4 \beta_{3}\right) S_{+} \vec{s}_{\omega}^{(-2)}-2\left(\rho_{3}+4 \beta_{3}\right) \vec{S}_{+} s_{\omega i}^{(-2) i}-2\left(\rho_{3}+4 \beta_{3}\right) \vec{s}^{(-2)} S_{+i}^{i} \\
& -4 \rho_{3} \vec{S}_{-} s^{(+1)}-2 \rho_{3} S_{-} \vec{s}^{(+1)}-2 \rho_{3} \vec{S}_{-} s_{i}^{(+1) i}-2 \rho_{3} \vec{s}^{(+1)} S_{-i}^{i} \\
& -4 \rho_{3} \vec{S}_{-} s^{(+2)}-2 \rho_{3} S_{-} \vec{s}^{(+2)}-2 \rho_{3} \vec{S}_{-} s_{i}^{(+2) i}-2 \rho_{3} \vec{s}^{(+2)} S_{-i}^{i} \\
& -4\left(\rho_{3}+4 \beta_{3}\right) \vec{S}_{-} s_{\omega}^{(+1)}-2\left(\rho_{3}+4 \beta_{3}\right) S_{-} \vec{s}_{\omega}^{(+1)}-2\left(\rho_{3}+4 \beta_{3}\right) \vec{S}_{-} s_{\omega i}^{(+1) i}-2\left(\rho_{3}+4 \beta_{3}\right) \vec{s}_{\omega}^{(+1)} S_{-i}^{i} \\
& -4\left(\rho_{3}+4 \beta_{3}\right) \vec{S}_{-} s_{\omega}^{(+2)}-2\left(\rho_{3}+4 \beta_{3}\right) S_{-} \vec{s}_{\omega}^{(+2)}-2\left(\rho_{3}+4 \beta_{3}\right) \vec{S}_{-} s_{\omega i}^{(+2) i}-2\left(\rho_{3}+4 \beta_{3}\right) \vec{s}^{(+2)} S_{-i}^{i}
\end{aligned}
$$

For $\mathcal{L}_{I}^{4 A}$ :

$$
\begin{aligned}
& \vec{S}_{I}^{4 \mathrm{~A}}=-2\left(\vec{e}^{[12]} \times \vec{b}^{[12]}\right)-2\left(\vec{e}^{[12]} \times \vec{b}^{[21]}+\vec{e}^{[21]} \times \vec{b}^{[12]}\right)+ \\
& -4\left(\vec{e}^{[13+]} \times \vec{b}^{[13-]}+\vec{e}^{[13-]} \times \vec{b}^{[13+]}\right)-4\left(\vec{e}^{[23+]} \times \vec{b}^{[23-]}+\vec{e}^{[23-]} \times \vec{b}^{[23+]}\right)+ \\
& -8\left(\vec{e}^{[13+]} \times \vec{b}^{[23-]}+\vec{e}^{[23-]} \times \vec{b}^{[13+]}\right)-4\left(\vec{e}^{[12]} \times \vec{b}^{[+-]}+\vec{e}^{[12]} \times \vec{b}^{[+-}\right)+ \\
& -16\left(\vec{e}^{(12)} \times \vec{b}_{\omega}^{(12)}+\vec{e}_{\omega}^{(12)} \times \vec{b}^{(12)}\right)-32\left(\vec{e}_{\omega}^{(12)} \times \vec{b}_{\omega}^{(12)}\right)+ \\
& -8\left(\vec{e}^{(13+)} \times \vec{b}^{(13-)}+\vec{e}^{(13-)} \times \vec{b}^{(13+)}\right)-8\left(\vec{e}^{(23+)} \times \vec{b}^{(23-)}+\vec{e}^{(23-)} \times \vec{b}^{(23+)}\right)+ \\
& -16\left(\vec{e}^{(13+)} \times \vec{b}^{(23-)}+\vec{e}^{(23-)} \times \vec{b}^{(23+)}\right)+8 i\left(\vec{e}^{(13+)} \times \vec{b}_{\omega}^{(24-)}+\vec{e}_{\omega}^{(24-)} \times \vec{b}^{(13+)}\right) \\
& -8 i\left(\vec{e}^{(13-)} \times \vec{b}_{\omega}^{(24+)}+\vec{e}_{\omega}^{(24+)} \times \vec{b}^{(13-)}\right)+8 i\left(\vec{e}^{(23+)} \times \vec{b}_{\omega}^{(14-)}+\vec{e}_{\omega}^{(14-)} \times \vec{b}^{(23+)}\right)+ \\
& +8 i\left(\vec{e}^{(23-)} \times \vec{b}_{\omega}^{(14+)}+\vec{e}_{\omega}^{(14+)} \times \vec{b}^{(23-)}\right)+32 i\left(\vec{e}_{\omega}^{(13+)} \times \vec{b}_{\omega}^{(24-)}+\vec{e}_{\omega}^{(24-)} \times \vec{b}_{\omega}^{(13+)}\right)+ \\
& -32 i\left(\vec{e}_{\omega}^{(13-)} \times \vec{b}_{\omega}^{(24+)}+\vec{e}_{\omega}^{(24+)} \times \vec{b}_{\omega}^{(13-)}\right)+32 i\left(\vec{e}_{\omega}^{(14-)} \times \vec{b}_{\omega}^{(23+)}+\vec{e}_{\omega}^{(23+)} \times \vec{b}_{\omega}^{(14-)}\right)+ \\
& -32 i\left(\vec{e}_{\omega}^{(14+)} \times \vec{b}_{\omega}^{(23-)}+\vec{e}_{\omega}^{(23-)} \times \vec{b}_{\omega}^{(14+)}\right)+4 i\left(\vec{e}^{(13-)} \times \vec{b}^{(24+)}+\vec{e}^{(24+)} \times \vec{b}^{(13-)}\right)+ \\
& -4 i\left(\vec{e}^{(13+)} \times \vec{b}^{(24-)}+\vec{e}^{(24-)} \times \vec{b}^{(13+)}\right)+8 i\left(\vec{e}^{(14+)} \times \vec{b}^{(23-)}+\vec{e}^{(23-)} \times \vec{b}^{(14+)}\right)+ \\
& -8 i\left(\vec{e}^{(14-)} \times \vec{b}^{(23+)}+\vec{e}^{(23+)} \times \vec{b}^{(14-)}\right)+8 i\left(\vec{e}^{(14-)} \times \vec{b}_{\omega}^{(23+)}+\vec{e}_{\omega}^{(23+)} \times \vec{b}^{(14-)}\right)+ \\
& -8 i\left(\vec{e}^{(14+)} \times \vec{b}_{\omega}^{(23-)}+\vec{e}_{\omega}^{(23-)} \times \vec{b}^{(14+)}\right)+8 i\left(\vec{e}^{(24-)} \times \vec{b}_{\omega}^{(13+)}+\vec{e}_{\omega}^{(13+)} \times \vec{b}^{(24-)}\right)+ \\
& -8 i\left(\vec{e}^{(24+)} \times \vec{b}_{\omega}^{(13-)}+\vec{e}_{\omega}^{(13-)} \times \vec{b}^{(24+)}\right)+16\left(\vec{e}^{(+-)} \times \vec{b}_{\omega}^{(+-)}+\vec{e}_{\omega}^{(+-)} \times \vec{b}^{(+-)}\right)
\end{aligned}
$$

For $\mathcal{L}_{I}^{4 S}$ : 


$$
\begin{aligned}
& \vec{S}_{I}^{4 S}=-4 s^{[12]} \vec{s}^{[12]}-4 \vec{s}^{[12]} \cdot \stackrel{\leftrightarrow}{s}^{[12]}-8 s^{[+-]} \vec{s}^{[+-]}-8 \vec{s}^{[+-]} \cdot \stackrel{\leftrightarrow}{s}^{[+-]}+
\end{aligned}
$$

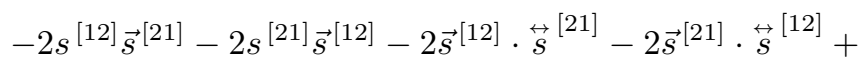

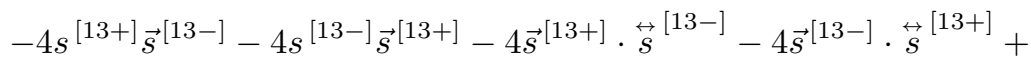

$$
\begin{aligned}
& -4 s^{[23+]} \vec{s}^{[23-]}-4 s^{[23-]} \vec{s}^{[23+]}-4 \vec{s}^{[23+]} \cdot \overleftrightarrow{s}^{[23-]}-4 \vec{s}^{[23-]} \cdot \overleftrightarrow{s}^{[23+]}+ \\
& -8 s^{[13+]} \vec{s}^{[23-]}-8 s^{[23-]} \vec{s}^{[13+]}-8 \vec{s}^{[13+]} \cdot \stackrel{\leftrightarrow}{s}^{[23-]}-8 \vec{s}^{[23-]} \cdot \stackrel{\leftrightarrow}{s}^{[13+]}+ \\
& -2 s^{(11)} \vec{s}^{(11)}-2 \vec{s}^{(11)} \cdot \stackrel{\leftrightarrow}{s}{ }^{(11)}-2 s^{(22)} \vec{s}^{(22)}-2 \vec{s}^{(22)} \cdot \stackrel{\leftrightarrow}{s}^{(22)}+ \\
& -2 s^{(11)} \vec{s}_{\omega}^{(11)}-2 s_{\omega}^{(11)} \vec{s}^{(11)}-2 \vec{s}^{(11)} \cdot \stackrel{\leftrightarrow}{s}_{\omega}^{(11)}-2 \vec{s}_{\omega}^{(11)} \cdot \stackrel{\leftrightarrow}{s}^{(11)}+ \\
& -2 s^{(22)} \vec{s}_{\omega}^{(22)}-2 s_{\omega}^{(22)} \vec{s}^{(22)}-2 \vec{s}^{(22)} \cdot \stackrel{\leftrightarrow}{s}_{\omega}^{(22)}-2 \vec{s}_{\omega}^{(22)} \cdot \stackrel{\leftrightarrow}{s}{ }^{(22)}+ \\
& -8 s_{\omega}^{(11)} \vec{s}_{\omega}^{(11)}-8 \vec{s}_{\omega}^{(11)} \cdot \stackrel{\leftrightarrow}{s}_{\omega}^{(11)}-8 s_{\omega}^{(22)} \vec{s}_{\omega}^{(22)}-8 \vec{s}_{\omega}^{(22)} \cdot \stackrel{\leftrightarrow}{s}_{\omega}^{(22)}+ \\
& -4 s^{(11)} \vec{s}^{(12)}-4 s^{(12)} \vec{s}^{(11)}-4 \vec{s}^{(11)} \cdot \overleftrightarrow{s}^{(12)}-4 \vec{s}^{(12)} \cdot \overleftrightarrow{s}^{(11)}+ \\
& -4 s^{(22)} \vec{s}^{(12)}-4 s^{(12)} \vec{s}^{(22)}-4 \vec{s}^{(22)} \cdot \stackrel{\leftrightarrow}{s}{ }^{(12)}-4 \vec{s}^{(12)} \cdot \stackrel{\leftrightarrow}{s}^{(22)}+ \\
& -8 s_{\omega}^{(11)} \vec{s}^{(12)}-8 s^{(12)} \vec{s}_{\omega}^{(11)}-8 \vec{s}_{\omega}^{(11)} \cdot \stackrel{\leftrightarrow}{s}{ }^{(12)}-8 \vec{s}^{(12)} \cdot \stackrel{\leftrightarrow}{s}_{\omega}^{(11)}+ \\
& -8 s_{\omega}^{(22)} \vec{s}^{(12)}-8 s^{(12)} \vec{s}_{\omega}^{(22)}-8 \vec{s}_{\omega}^{(22)} \cdot \stackrel{\leftrightarrow}{s}{ }^{(12)}-8 \vec{s}^{(12)} \cdot \stackrel{\leftrightarrow}{s}_{\omega}^{(22)}+ \\
& -16 s_{\omega}^{(11)} \vec{s}_{\omega}^{(12)}-16 s^{(12)} \vec{s}_{\omega}^{(11)}-16 \vec{s}_{\omega}^{(11)} \cdot \stackrel{\leftrightarrow}{s}_{\omega}^{(12)}-16 \vec{s}_{\omega}^{(12)} \cdot \stackrel{\leftrightarrow}{s}_{\omega}^{(11)}+ \\
& -16 s_{\omega}^{(22)} \vec{s}_{\omega}^{(12)}-16 s_{\omega}^{(12)} \vec{s}_{\omega}^{(22)}-16 \vec{s}_{\omega}^{(22)} \cdot \stackrel{\leftrightarrow}{s}_{\omega}^{(12)}-16 \vec{s}_{\omega}^{(12)} \cdot \stackrel{\leftrightarrow}{s}_{\omega}^{(22)}+ \\
& -2 s^{(11)} \vec{s}^{(22)}-2 s^{(22)} \vec{s}^{(11)}-2 \vec{s}^{(11)} \cdot \stackrel{\leftrightarrow}{s}^{(22)}-2 \vec{s}^{(22)} \cdot \stackrel{\leftrightarrow}{s}^{(11)}+
\end{aligned}
$$

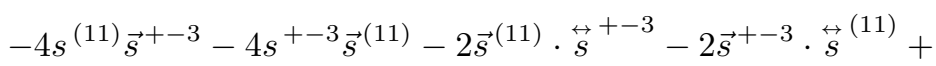

$$
\begin{aligned}
& -4 s^{(22)} \vec{s}^{+-3}-4 s^{+-3} \vec{s}^{(22)}-2 \vec{s}^{(22)} \cdot \stackrel{\leftrightarrow}{s}^{+-3}-2 \vec{s}^{+-3} \cdot \stackrel{\leftrightarrow}{s}^{(22)}+ \\
& -8 s^{(12)} \vec{s}^{(12)}-8 \vec{s}^{(12)} \cdot \stackrel{\leftrightarrow}{s}^{(12)}+ \\
& -8 s^{(12)} \vec{s}_{\omega}^{(12)}-8 s_{\omega}^{(12)} \vec{s}^{(12)}-8 \vec{s}^{(12)} \cdot \stackrel{\leftrightarrow}{s}_{\omega}^{(12)}-8 \vec{s}_{\omega}^{(12)} \cdot \stackrel{\leftrightarrow}{s}^{(12)}+ \\
& -16 s_{\omega}^{(12)} \vec{s}_{\omega}^{(12)}-8 \vec{s}_{\omega}^{(12)} \cdot \stackrel{\leftrightarrow}{s}_{\omega}^{(12)}+ \\
& -4 s^{(13+)} \vec{s}^{(13-)}-4 s^{(13-)} \vec{s}^{(13+)}-4 \vec{s}^{(13+)} \cdot \stackrel{\leftrightarrow}{s}^{(13-)}-4 \vec{s}^{(13-)} \cdot \overleftrightarrow{s}^{(13+)}+ \\
& -4 s^{(23+)} \vec{s}^{(23-)}-4 s^{(23-)} \vec{s}^{(23+)}-4 \vec{s}^{(23+)} \cdot \stackrel{\leftrightarrow}{s}^{(23-)}-4 \vec{s}^{(23-)} \cdot \stackrel{\leftrightarrow}{s}^{(23+)}+
\end{aligned}
$$

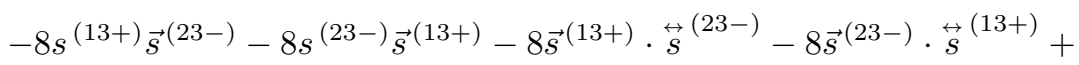

$$
\begin{aligned}
& -8 s^{(12)} \vec{s}^{+-3}-8 s^{+-3} \vec{s}^{(12)}-8 \vec{s}^{(12)} \cdot \overleftrightarrow{s}^{+-3}-8 \vec{s}^{+-3} \cdot \stackrel{\leftrightarrow}{s}^{(12)}+ \\
& -4 s^{+-3} \vec{s}^{+-4}-4 s^{+-4} \vec{s}^{+-3}-4 \vec{s}^{+-3} \cdot \stackrel{\leftrightarrow}{s}^{+-4}-4 \vec{s}^{+-4} \cdot \stackrel{\leftrightarrow}{s}^{+-3}+ \\
& -4 i s^{(13+)} \vec{s}_{\omega}^{(24-)}-4 i s_{\omega}^{(24-)} \vec{s}^{(13+)}-4 i \vec{s}^{(13+)} \cdot \stackrel{\leftrightarrow}{s}_{\omega}^{(24-)}-4 i \vec{s}^{(13+)} \cdot \stackrel{\leftrightarrow}{s}_{\omega}^{(24-)}+ \\
& +4 i s^{(13-)} \vec{s}_{\omega}^{(24+)}+4 i s_{\omega}^{(24+)} \vec{s}^{(13-)}+4 i \vec{s}^{(13-)} \cdot \stackrel{\leftrightarrow}{s}_{\omega}^{(24+)}-4 i \vec{s}^{(13-)} \cdot \stackrel{\leftrightarrow}{s}_{\omega}^{(24+)}+ \\
& -4 i s^{(23+)} \vec{s}_{\omega}^{(14-)}-4 i s_{\omega}^{(14-)} \vec{s}^{(23+)}-4 i \vec{s}^{(23+)} \cdot \stackrel{\leftrightarrow}{s}_{\omega}^{(14-)}-4 i \vec{s}^{(23+)} \cdot \stackrel{\leftrightarrow}{s}_{\omega}^{(14-)}+ \\
& +4 i s^{(23-)} \vec{s}_{\omega}^{(14+)}+4 i s_{\omega}^{(14+)} \vec{s}^{(23-)}+4 i \vec{s}^{(23-)} \cdot \stackrel{\leftrightarrow}{s}_{\omega}^{(14+)}-4 i \vec{s}^{(23-)} \cdot \stackrel{\leftrightarrow}{s}_{\omega}^{(14+)}+
\end{aligned}
$$




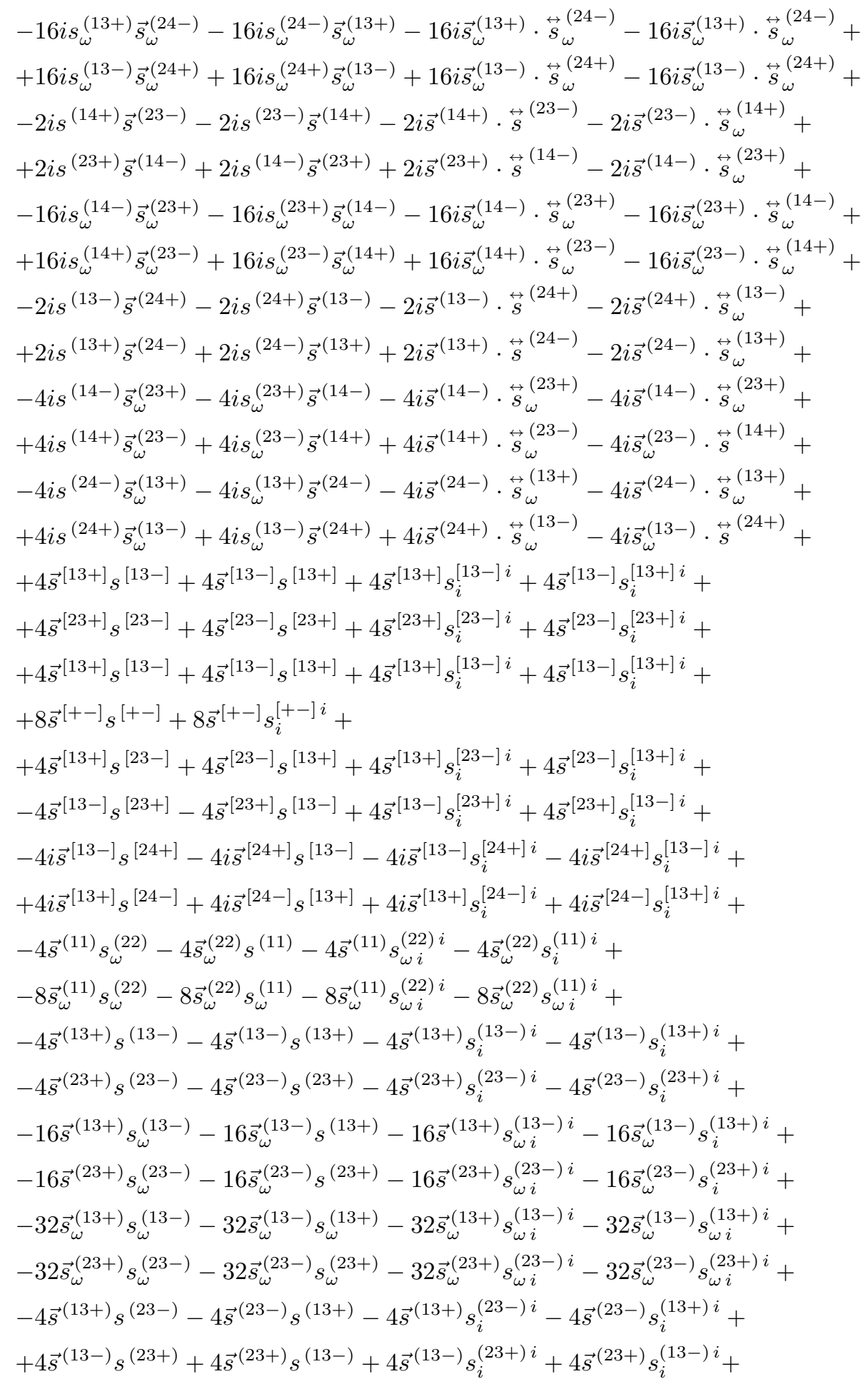




$$
\begin{aligned}
& -16 \vec{s}^{(13+)} s_{\omega}^{(13-)}-16 \vec{s}_{\omega}^{(13-)} s^{(13+)}-16 \vec{s}^{(13+)} s_{\omega i}^{(13-) i}-16 \vec{s}_{\omega}^{(13-)} s_{i}^{(13+) i}+ \\
& +16 \vec{s}^{(13-)} s_{\omega}^{(13+)}+16 \vec{s}_{\omega}^{(13+)} s^{(13-)}+16 \vec{s}^{(13-)} s_{\omega i}^{(13+) i}+16 \vec{s}_{\omega}^{(13+)} s_{i}^{(13-) i}+ \\
& -32 \vec{s}_{\omega}^{(13+)} s_{\omega}^{(13-)}-32 \vec{s}_{\omega}^{(13-)} s_{\omega}^{(13+)}-32 \vec{s}_{\omega}^{(13+)} s_{\omega i}^{(13-) i}-32 \vec{s}_{\omega}^{(13-)} s_{\omega i}^{(13+) i}+ \\
& +32 \vec{s}_{\omega}^{(13-)} s_{\omega}^{(13+)}+32 \vec{s}_{\omega}^{(13+)} s_{\omega}^{(13-)}+32 \vec{s}_{\omega}^{(13-)} s_{\omega i}^{(13+) i}+32 \vec{s}_{\omega}^{(13+)} s_{\omega i}^{(13-) i}+ \\
& +8 \vec{s}^{(+-)} s^{(+-)}+8 \vec{s}^{(+-)} s_{i}^{(+-) i}+ \\
& -8 \vec{s}^{+-3} s_{\omega}^{+-4}-8 \vec{s}_{\omega}^{+-4} s^{+-3}-8 \vec{s}^{+-3} s_{\omega i}^{+-4 i}-8 \vec{s}_{\omega}^{+-4} s_{i}^{+-3 i}+ \\
& -16 \vec{s}_{\omega}^{+-3} s_{\omega}^{+-4}-16 \vec{s}_{\omega}^{+-4} s_{\omega}^{+-3}-16 \vec{s}_{\omega}^{+-3} s_{\omega i}^{+-4 i}-16 \vec{s}_{\omega}^{+-4} s_{\omega i}^{+-3 i}+ \\
& -8 \vec{s}^{(11)} s_{\omega}^{+-3}-8 \vec{s}_{\omega}^{+-3} s^{(11)}-8 \vec{s}^{(11)} s_{\omega i}^{+-3 i}-8 \vec{s}_{\omega}^{+-3} s_{i}^{(11) i}+ \\
& -8 \vec{s}^{(22)} s_{\omega}^{+-3}-8 \vec{s}_{\omega}^{+-3} s^{(22)}-8 \vec{s}^{(22} s_{\omega i}^{+-3 i}-8 \vec{s}_{\omega}^{+-3} s_{i}^{(22) i}+ \\
& -16 \vec{s}_{\omega}^{(11)} s_{\omega}^{+-3}-16 \vec{s}_{\omega}^{+-3} s_{\omega}^{(11)}-16 \vec{s}_{\omega}^{(11)} s_{\omega i}^{+-3 i}-16 \vec{s}_{\omega}^{+-3} s_{\omega i}^{(11) i}+ \\
& -16 \vec{s}_{\omega}^{(22)} s_{\omega}^{+-3}-16 \vec{s}_{\omega}^{+-3} s_{\omega}^{(22)}-16 \vec{s}_{\omega}^{(22)} s_{\omega i}^{+-3 i}-16 \vec{s}_{\omega}^{+-3} s_{\omega i}^{(22) i}+ \\
& -16 \vec{s}^{(12)} s_{\omega}^{+-3}-16 \vec{s}_{\omega}^{+-3} s^{(12)}-16 \vec{s}^{(12)} s_{\omega i}^{+-3 i}-16 \vec{s}_{\omega}^{+-3} s_{i}^{(12) i}+ \\
& -32 \vec{s}_{\omega}^{(12)} s_{\omega}^{+-3}-32 \vec{s}_{\omega}^{+-3} s_{\omega}^{(12)}-8 \vec{s}_{\omega}^{(12)} s_{\omega i}^{+-3 i}-8 \vec{s}_{\omega}^{+-3} s_{\omega i}^{(12) i}
\end{aligned}
$$

The above expressions are extending the Poynting vector physics. Showing on the presence of an EM flux propagation larger than Maxwell supposes. Enlarging the literature [23].

\section{Stress Tensor in Vectorial Form}

The global stress tensor of the system is

$$
T^{i j}=T_{K}^{i j}+T_{M}^{i j}+\vec{T}_{G F}^{i j}+T_{I}^{i j}
$$

For $\mathcal{L}_{K}^{A}$ :

$$
\begin{aligned}
& T_{K i j}^{A}=a_{1}\left(\left|\vec{E}_{A}\right|^{2}+\left|\vec{B}_{A}\right|^{2}\right) \delta_{i j}-2 a_{1}\left(\vec{E}_{A i} \vec{E}_{A j}+\vec{B}_{A i} \vec{B}_{A j}\right)+ \\
& +a_{2}\left(\left|\vec{E}_{U}\right|^{2}+\left|\vec{B}_{U}\right|^{2}\right) \delta_{i j}-2 a_{2}\left(\vec{E}_{U i} \vec{E}_{U j}+\vec{B}_{U i} \vec{B}_{U j}\right) \\
& +2 a_{3}\left(\vec{E}_{+} \vec{E}_{-}+\vec{B}_{+} \vec{B}_{-}\right) \delta_{i j}-4 a_{1}\left(\vec{E}_{+i} \vec{E}_{-j}+\vec{B}_{+i} \vec{B}_{-j}\right)
\end{aligned}
$$

For $\mathcal{L}_{K}^{S}$ : 


$$
\begin{aligned}
& T_{K i j}^{S}=-\frac{1}{2} b_{(11)}\left(S_{A}^{2}+\left|\vec{S}_{A}\right|^{2}+\overleftrightarrow{S}_{A} \cdot \stackrel{\leftrightarrow}{S}_{A}\right) \delta_{i j}-2 b_{(11)} \vec{S}_{A i} \vec{S}_{A j}-2 b_{(11)} S_{A i k} S_{A j}^{k} \\
& -\frac{1}{2} b_{(22)}\left(S_{U}^{2}+\left|\vec{S}_{U}\right|^{2}+\stackrel{\leftrightarrow}{S}_{U} \cdot \stackrel{\leftrightarrow}{S}_{U}\right) \delta_{i j}-2 b_{(22)} \vec{S}_{U i} \vec{S}_{U j}-2 b_{(11)} S_{U i k} S_{U j}^{k}+ \\
& -b_{(33)}\left(S_{+} S_{-}+\vec{S}_{+} \cdot \vec{S}_{-}+\stackrel{\leftrightarrow}{S}_{+} \cdot \stackrel{\leftrightarrow}{S}_{-}\right) \delta_{i j}+ \\
& -2 b_{(33)} \vec{S}_{+i} \vec{S}_{-j}-2 b_{(33)} \vec{S}_{-i} \vec{S}_{+j}+2 b_{(33)} S_{+i k} S_{-k j}+2 b_{(33)} S_{-i k} S_{+k j}+ \\
& +\frac{c_{(11)}}{2}\left(S_{A}+S_{k A}^{k}\right)^{2} \delta_{i j}-2 c_{(11)} S_{A i j}\left(S_{A}+S_{k A}^{k}\right)+ \\
& +\frac{c_{(22)}}{2}\left(S_{U}+S_{k U}^{k}\right)^{2} \delta_{i j}-2 c_{(22)} S_{U i j}\left(S_{U}+S_{k U}^{k}\right)+ \\
& +c_{(33)}\left(S_{+}+S_{k+}^{k}\right)\left(S_{-}+S_{l-}^{l}\right) \delta_{i j}-2 c_{(33)} S_{+i j}\left(S_{-}+S_{k-}^{k}\right)-2 c_{(33)} S_{-i j}\left(S_{+}+S_{k+}^{k}\right)
\end{aligned}
$$

For $\mathcal{L}_{M}$ :

$$
\begin{aligned}
& T_{M i j}=\frac{1}{4} m_{U}^{2}\left(\phi_{U}^{2}-|\vec{U}|^{2}\right) \delta_{i j}+\frac{1}{2} m_{U}^{2} \vec{U}_{i} \vec{U}_{j}+ \\
& +\frac{1}{2} m_{ \pm}^{2}\left(\phi_{+} \phi_{-}-\vec{V}_{+} \cdot \vec{V}_{-}\right) \delta_{i j}+\frac{1}{2} m_{ \pm}^{2}\left(\vec{V}_{i} \vec{V}_{j}+\vec{V}_{i} \vec{V}_{j}\right)
\end{aligned}
$$

For $\mathcal{L}_{G F}$ :

$$
\begin{aligned}
& T_{\mathrm{GF} i j}^{A}=\frac{\xi_{(11)}}{8}\left(S_{A}+S_{A k}^{k}\right)^{2} \delta_{i j}-\frac{1}{2} \xi_{(11)} S_{A i j}\left(S_{A}+S_{A k}^{k}\right)+ \\
& +\frac{\xi_{(22)}}{8}\left(S_{U}+S_{U k}^{k}\right)^{2} \delta_{i j}-\frac{1}{2} \xi_{(22)} S_{U i j}\left(S_{U}+S_{U k}^{k}\right)+ \\
& +\frac{\xi_{(12)}}{4}\left(S_{A}+S_{A k}^{k}\right)\left(S_{U}+S_{U l}^{l}\right) \delta_{i j}-\xi_{(12)} S_{A i j}\left(S_{U}+S_{U k}^{k}\right)-\xi_{(12)} S_{U i j}\left(S_{A}+S_{A k}^{k}\right)+ \\
& +\frac{\xi_{(33)}}{4}\left(S_{+}+S_{+k}^{k}\right)\left(S_{-}+S_{-l}^{l}\right) \delta_{i j}-\xi_{(33)} S_{+i j}\left(S_{-}+S_{-k}^{k}\right)-\xi_{(33)} S_{-i j}\left(S_{+}+S_{+k}^{k}\right)
\end{aligned}
$$

For $\mathcal{L}_{I}^{3 A}$ : 


$$
\begin{aligned}
& T_{I}^{3 A}{ }_{i j}=4 b_{1}\left(\vec{E}_{A} \cdot \vec{e}^{[12]}+\vec{B}_{A} \cdot \vec{b}^{[12]}\right) \delta_{i j}-8 b_{1}\left(\vec{E}_{A i} \vec{e}_{j}^{[12]}+\vec{e}_{i}^{[12]} \vec{E}_{A j}+\vec{B}_{A i} \vec{b}_{j}^{[12]}+\vec{b}_{i}^{[12]} \vec{B}_{A j}\right) \\
& +4 b_{2}\left(\vec{E}_{U} \cdot \vec{e}^{[12]}+\vec{B}_{U} \cdot \vec{b}^{[12]}\right) \delta_{i j}-8 b_{2}\left(\vec{E}_{U i} \vec{e}_{j}^{[12]}+\vec{e}_{i}^{[12]} \vec{E}_{U j}+\vec{B}_{U i} \vec{b}_{j}^{[12]}+\vec{b}_{i}^{[12]} \vec{B}_{U j}\right)+ \\
& +4 b_{1}\left(\vec{E}_{A} \cdot \vec{e}^{[+-]}+\vec{B}_{A} \cdot \vec{b}^{[+-]}\right) \delta_{i j}-8 b_{1}\left(\vec{E}_{A i} \vec{e}_{j}^{[+-]}+\vec{e}_{i}^{[+-]} \vec{E}_{A j}+\vec{B}_{A i} \vec{b}_{j}^{[+-]}+\vec{b}_{i}^{[+-]} \vec{B}_{A j}\right)+ \\
& +4 b_{2}\left(\vec{E}_{U} \cdot \vec{e}^{[+-]}+\vec{B}_{U} \cdot \vec{b}^{[+-]}\right) \delta_{i j}-8 b_{2}\left(\vec{E}_{U i} \vec{e}_{j}^{[+-]}+\vec{e}_{i}^{[+-]} \vec{E}_{U j}+\vec{B}_{U i} \vec{b}_{j}^{[+-]}+\vec{b}_{i}^{[+-]} \vec{B}_{U j}\right)+ \\
& +4 \beta_{1}\left(\vec{E}_{A} \cdot \vec{e}^{(+-)}+\vec{B}_{A} \cdot \vec{b}^{(+-)}\right) \delta_{i j}-8 \beta_{1}\left(\vec{E}_{A i} \vec{e}_{j}^{(+-)}+\vec{e}_{i}^{(+-)} \vec{E}_{A j}+\vec{B}_{A i} \vec{b}_{j}^{(+-)}+\vec{b}_{i}^{(+-)} \vec{B}_{A j}\right)+ \\
& +4 \beta_{2}\left(\vec{E}_{U} \cdot \vec{e}^{(+-)}+\vec{B}_{U} \cdot \vec{b}^{(+-)}\right) \delta_{i j}-8 \beta_{2}\left(\vec{E}_{U i} \vec{e}_{j}^{(+-)}+\vec{e}_{i}^{(+-)} \vec{E}_{U j}+\vec{B}_{U i} \vec{b}_{j}^{(+-)}+\vec{b}_{i}^{(+-)} \vec{B}_{U j}\right)+ \\
& +4 b_{3}\left(\vec{E}_{+} \cdot \vec{e}^{[-1]}+\vec{B}_{+} \cdot \vec{b}^{[-1]}\right) \delta_{i j}-8 b_{3}\left(\vec{E}_{+i} \vec{e}_{j}^{[-1]}+\vec{e}_{i}^{[-1]} \vec{E}_{+j}+\vec{B}_{+i} \vec{b}_{j}^{[-1]}+\vec{b}_{i}^{[-1]} \vec{B}_{+j}\right)+ \\
& +4 b_{3}\left(\vec{E}_{+} \cdot \vec{e}^{[-2]}+\vec{B}_{+} \cdot \vec{b}^{[-2]}\right) \delta_{i j}-8 b_{3}\left(\vec{E}_{+i} \vec{e}_{j}^{[-2]}+\vec{e}_{i}^{[-2]} \vec{E}_{+j}+\vec{B}_{+i} \vec{b}_{j}^{[-2]}+\vec{b}_{i}^{[-2]} \vec{B}_{+j}\right)+ \\
& +4 b_{3}\left(\vec{E}_{-} \cdot \vec{e}^{[+1]}+\vec{B}_{-} \cdot \vec{b}^{[+1]}\right) \delta_{i j}-8 b_{3}\left(\vec{E}_{-i} \vec{e}_{j}^{[+1]}+\vec{e}_{i}^{[+1]} \vec{E}_{-j}+\vec{B}_{-i} \vec{b}_{j}^{[+1]}+\vec{b}_{i}^{[+1]} \vec{B}_{-j}\right)+ \\
& +4 b_{3}\left(\vec{E}_{-} \cdot \vec{e}^{[+2]}+\vec{B}_{-} \cdot \vec{b}^{[+2]}\right) \delta_{i j}-8 b_{3}\left(\vec{E}_{-i} \vec{e}_{j}^{[+2]}+\vec{e}_{i}^{[+2]} \vec{E}_{-j}+\vec{B}_{-i} \vec{b}_{j}^{[+2]}+\vec{b}_{i}^{[+2]} \vec{B}_{-j}\right)
\end{aligned}
$$

For $\mathcal{L}_{I}^{3 S}$ 


$$
\begin{aligned}
& T_{I}^{3 S}{ }_{i j}=-2 \beta_{1} \vec{S}_{1 i} \vec{s}_{j}^{(11)}-2 \beta_{1} \vec{s}_{i}^{(11)} \vec{S}_{1 j}+2 \beta_{1} S_{1 i k} s_{k j}^{(11)}+2 \beta_{1} s_{i k}^{(11)} S_{1 k j}+ \\
& -2 \beta_{1} \vec{S}_{1 i} \vec{s}_{j}^{(22)}-2 \beta_{1} \vec{s}_{i}^{(22)} \vec{S}_{1 j}+2 \beta_{1} S_{1 i k} s_{k j}^{(22)}+2 \beta_{1} s_{i k}^{(22)} S_{1 k j}+ \\
& -4 \beta_{1} \vec{S}_{1 i} \vec{s}_{j}^{(12)}-4 \beta_{1} \vec{s}_{i}^{(12)} \vec{S}_{1 j}+4 \beta_{1} S_{1 i k} s_{k j}^{(12)}+4 \beta_{1} s_{i k}^{(12)} S_{1 k j}+ \\
& -4 \beta_{1} \vec{S}_{1 i} \vec{s}_{j}^{+-3}-4 \beta_{1} \vec{s}_{i}^{+-3} \vec{S}_{1 j}+4 \beta_{1} S_{1 i k} s_{k j}^{+-3}+4 \beta_{1} s_{i k}^{+-3} S_{1 k j}+ \\
& -2 \beta_{2} \vec{S}_{2 i} \vec{s}_{j}^{(11)}-2 \beta_{2} \vec{s}_{i}^{(11)} \vec{S}_{2 j}+2 \beta_{2} S_{2 i k} s_{k j}^{(11)}+2 \beta_{2} s_{i k}^{(11)} S_{2 k j}+ \\
& -2 \beta_{2} \vec{S}_{2 i} \vec{s}_{j}^{(22)}-2 \beta_{2} \vec{s}_{i}^{(22)} \vec{S}_{2 j}+2 \beta_{2} S_{2 i k} s_{k j}^{(22)}+2 \beta_{2} s_{i k}^{(22)} S_{2 k j}+ \\
& -4 \beta_{2} \vec{S}_{2 i} \vec{s}_{j}^{(12)}-4 \beta_{2} \vec{s}_{i}^{(12)} \vec{S}_{2 j}+4 \beta_{2} S_{2 i k} s_{k j}^{(12)}+4 \beta_{2} s_{i k}^{(12)} S_{2 k j}+ \\
& -4 \beta_{2} \vec{S}_{2 i} \vec{s}_{j}^{+-3}-4 \beta_{2} \vec{s}_{i}^{+-3} \vec{S}_{2 j}+4 \beta_{2} S_{2 i k} s_{k j}^{+-3}+4 \beta_{2} s_{i k}^{+-3} S_{2 k j}+ \\
& -4 \beta_{3} \vec{S}_{+i} \vec{s}_{j}^{(-1)}-4 \beta_{3} \vec{s}_{i}^{(-1)} \vec{S}_{+j}+4 \beta_{2} S_{+i k} s_{k j}^{(-1)}+4 \beta_{3} s_{i k}^{(-1)} S_{+k j}+ \\
& -4 \beta_{2} \vec{S}_{+i} \vec{s}_{j}^{(-2)}-4 \beta_{3} \vec{s}_{i}^{(-2)} \vec{S}_{+j}+4 \beta_{2} S_{+i k} s_{k j}^{(-2)}+4 \beta_{3} s_{i k}^{(-2)} S_{+k j}+ \\
& -4 \beta_{3} \vec{S}_{-i} \vec{s}_{j}^{(+1)}-4 \beta_{3} \vec{s}_{i}^{(+1)} \vec{S}_{-j}+4 \beta_{2} S_{-i k} s_{k j}^{(+1)}+4 \beta_{3} s_{i k}^{(+1)} S_{-k j}+ \\
& -4 \beta_{2} \vec{S}_{-i} \vec{s}_{j}^{(+2)}-4 \beta_{3} \vec{s}_{i}^{(+2)} \vec{S}_{-j}+4 \beta_{2} S_{-i k} s_{k j}^{(+2)}+4 \beta_{3} s_{i k}^{(+2)} S_{-k j}+ \\
& +\rho_{1}\left(S_{1}+S_{1 k}^{k}\right)\left(s^{(11)}+s_{l}^{(11) l}\right) \delta_{i j}-2 \rho_{1} S_{1 i j}\left(s^{(11)}+s_{k}^{(11) k}\right)-2 \rho_{1} s_{i j}^{(11)}\left(S_{1}+S_{1 k}^{k}\right) \\
& +\rho_{1}\left(S_{1}+S_{1 k}^{k}\right)\left(s^{(22)}+s_{l}^{(22) l}\right) \delta_{i j}-2 \rho_{1} S_{1 i j}\left(s^{(22)}+s_{k}^{(22) k}\right)-2 \rho_{1} s_{i j}^{(22)}\left(S_{1}+S_{1 k}^{k}\right) \\
& +2 \rho_{1}\left(S_{1}+S_{1 k}^{k}\right)\left(s^{(12)}+s_{l}^{(12) l}\right) \delta_{i j}-4 \rho_{1} S_{1 i j}\left(s^{(12)}+s_{k}^{(12) k}\right)-4 \rho_{1} s_{i j}^{(12)}\left(S_{1}+S_{1 k}^{k}\right) \\
& +2 \rho_{1}\left(S_{1}+S_{1 k}^{k}\right)\left(s^{+-3}+s_{l}^{+-3 l}\right) \delta_{i j}-4 \rho_{1} S_{1 i j}\left(s^{+-3}+s_{k}^{+-3 k}\right)-4 \rho_{1} s_{i j}^{+-3}\left(S_{1}+S_{1 k}^{k}\right) \\
& +\left(\rho_{1}+4 \beta_{1}\right)\left(S_{1}+S_{1 k}^{k}\right)\left(s_{\omega}^{(11)}+s_{\omega l}^{(11) l}\right) \delta_{i j}+ \\
& -2\left(\rho_{1}+4 \beta_{1}\right) S_{1 i j}\left(s_{\omega}^{(11)}+s_{\omega k}^{(11) k}\right)-2\left(\rho_{1}+4 \beta_{1}\right) s_{\omega i j}^{(11)}\left(S_{1}+S_{1 k}^{k}\right) \\
& +\left(\rho_{1}+4 \beta_{1}\right)\left(S_{1}+S_{1 k}^{k}\right)\left(s_{\omega}^{(22)}+s_{\omega l}^{(22) l}\right) \delta_{i j}+ \\
& -2\left(\rho_{1}+4 \beta_{1}\right) S_{1 i j}\left(s_{\omega}^{(22)}+s_{\omega k}^{(22) k}\right)-2\left(\rho_{1}+4 \beta_{1}\right) s_{\omega i j}^{(22)}\left(S_{1}+S_{1 k}^{k}\right) \\
& +2\left(\rho_{1}+4 \beta_{1}\right)\left(S_{1}+S_{1 k}^{k}\right)\left(s_{\omega}^{(12)}+s_{\omega l}^{(12) l}\right) \delta_{i j}+ \\
& -4\left(\rho_{1}+4 \beta_{1}\right) S_{1 i j}\left(s_{\omega}^{(12)}+s_{\omega k}^{(12) k}\right)-4\left(\rho_{1}+4 \beta_{1}\right) s_{\omega i j}^{(12)}\left(S_{1}+S_{1 k}^{k}\right) \\
& +2\left(\rho_{1}+4 \beta_{1}\right)\left(S_{1}+S_{1 k}^{k}\right)\left(s_{\omega}^{+-3}+s_{\omega l}^{+-3 l}\right) \delta_{i j}+ \\
& -4\left(\rho_{1}+4 \beta_{1}\right) S_{1 i j}\left(s_{\omega}^{+-3}+s_{\omega k}^{+-3 k}\right)-4\left(\rho_{1}+4 \beta_{1}\right) s_{\omega i j}^{+-3}\left(S_{1}+S_{1 k}^{k}\right)
\end{aligned}
$$




$$
\begin{aligned}
& +\rho_{2}\left(S_{2}+S_{2 k}^{k}\right)\left(s^{(11)}+s_{l}^{(11) l}\right) \delta_{i j}-2 \rho_{2} S_{2 i j}\left(s^{(11)}+s_{k}^{(11) k}\right)-2 \rho_{2} s_{i j}^{(11)}\left(S_{2}+S_{2 k}^{k}\right) \\
& +\rho_{2}\left(S_{2}+S_{2 k}^{k}\right)\left(s^{(22)}+s_{l}^{(22) l}\right) \delta_{i j}-2 \rho_{2} S_{2 i j}\left(s^{(22)}+s_{k}^{(22) k}\right)-2 \rho_{2} s_{i j}^{(22)}\left(S_{2}+S_{2 k}^{k}\right) \\
& +2 \rho_{2}\left(S_{2}+S_{2 k}^{k}\right)\left(s^{(12)}+s_{l}^{(12) l}\right) \delta_{i j}-4 \rho_{2} S_{2 i j}\left(s^{(12)}+s_{k}^{(12) k}\right)-4 \rho_{2} s_{i j}^{(12)}\left(S_{2}+S_{2 k}^{k}\right) \\
& +2 \rho_{2}\left(S_{2}+S_{2 k}^{k}\right)\left(s^{+-3}+s_{l}^{+-3 l}\right) \delta_{i j}-4 \rho_{2} S_{2 i j}\left(s^{+-3}+s_{k}^{+-3 k}\right)-4 \rho_{2} s_{i j}^{+-3}\left(S_{2}+S_{2 k}^{k}\right) \\
& +\left(\rho_{2}+4 \beta_{2}\right)\left(S_{2}+S_{2 k}^{k}\right)\left(s_{\omega}^{(11)}+s_{\omega l}^{(11) l}\right) \delta_{i j}+ \\
& -2\left(\rho_{2}+4 \beta_{2}\right) S_{2 i j}\left(s_{\omega}^{(11)}+s_{\omega k}^{(11) k}\right)-2\left(\rho_{2}+4 \beta_{2}\right) s_{\omega i j}^{(11)}\left(S_{2}+S_{2 k}^{k}\right) \\
& +\left(\rho_{2}+4 \beta_{2}\right)\left(S_{2}+S_{2 k}^{k}\right)\left(s_{\omega}^{(22)}+s_{\omega l}^{(22) l}\right) \delta_{i j}+ \\
& -2\left(\rho_{2}+4 \beta_{2}\right) S_{2 i j}\left(s_{\omega}^{(22)}+s_{\omega k}^{(22) k}\right)-2\left(\rho_{2}+4 \beta_{2}\right) s_{\omega i j}^{(22)}\left(S_{2}+S_{2 k}^{k}\right) \\
& +2\left(\rho_{2}+4 \beta_{2}\right)\left(S_{2}+S_{2 k}^{k}\right)\left(s_{\omega}^{(12)}+s_{\omega l}^{(12) l}\right) \delta_{i j}+ \\
& -4\left(\rho_{2}+4 \beta_{2}\right) S_{2 i j}\left(s_{\omega}^{(12)}+s_{\omega k}^{(12) k}\right)-4\left(\rho_{2}+4 \beta_{2}\right) s_{\omega i j}^{(12)}\left(S_{2}+S_{2 k}^{k}\right) \\
& +2\left(\rho_{2}+4 \beta_{2}\right)\left(S_{2}+S_{2 k}^{k}\right)\left(s_{\omega}^{+-3}+s_{\omega l}^{+-3 l}\right) \delta_{i j}+ \\
& -4\left(\rho_{2}+4 \beta_{2}\right) S_{2 i j}\left(s_{\omega}^{+-3}+s_{\omega k}^{+-3 k}\right)-4\left(\rho_{2}+4 \beta_{2}\right) s_{\omega i j}^{+-3}\left(S_{2}+S_{2 k}^{k}\right) \\
& +\rho_{3}\left(S_{+}+S_{+k}^{k}\right)\left(s^{(-1)}+s_{l}^{(-1) l}\right) \delta_{i j}-2 \rho_{3} S_{+i j}\left(s^{(-1)}+s_{k}^{(-1) k}\right)-2 \rho_{3} s_{i j}^{(-1)}\left(S_{+}+S_{+k}^{k}\right) \\
& +\rho_{3}\left(S_{+}+S_{+k}^{k}\right)\left(s^{(-2)}+s_{l}^{(-2) l}\right) \delta_{i j}-2 \rho_{3} S_{+i j}\left(s^{(-2)}+s_{k}^{(-1) k}\right)-2 \rho_{3} s_{i j}^{(-2)}\left(S_{+}+S_{+k}^{k}\right) \\
& +\rho_{3}\left(S_{-}+S_{-k}^{k}\right)\left(s^{(+1)}+s_{l}^{(+1) l}\right) \delta_{i j}-2 \rho_{3} S_{-i j}\left(s^{(+1)}+s_{k}^{(+1) k}\right)-2 \rho_{3} s_{i j}^{(+1)}\left(S_{-}+S_{-k}^{k}\right) \\
& +\rho_{3}\left(S_{-}+S_{-k}^{k}\right)\left(s^{(-2)}+s_{l}^{(+2) l}\right) \delta_{i j}-2 \rho_{3} S_{-i j}\left(s^{(+2)}+s_{k}^{(+1) k}\right)-2 \rho_{3} s_{i j}^{(+2)}\left(S_{-}+S_{-k}^{k}\right) \\
& +\left(\rho_{3}+4 \beta_{3}\right)\left(S_{+}+S_{+k}^{k}\right)\left(s_{\omega}^{(-1)}+s_{\omega l}^{(-1) l}\right) \delta_{i j}+ \\
& -2\left(\rho_{3}+4 \beta_{3}\right) S_{+i j}\left(s_{\omega}^{(-1)}+s_{\omega k}^{(-1) k}\right)-2\left(\rho_{3}+4 \beta_{3}\right) s_{\omega i j}^{(-1)}\left(S_{+}+S_{+k}^{k}\right) \\
& +\left(\rho_{3}+4 \beta_{3}\right)\left(S_{+}+S_{+k}^{k}\right)\left(s_{\omega}^{(-2)}+s_{\omega l}^{(-2) l}\right) \delta_{i j}+ \\
& -2\left(\rho_{3}+4 \beta_{3}\right) S_{+i j}\left(s_{\omega}^{(-2)}+s_{\omega k}^{(-1) k}\right)-2\left(\rho_{3}+4 \beta_{3}\right) s_{\omega i j}^{(-2)}\left(S_{+}+S_{+k}^{k}\right) \\
& +\left(\rho_{3}+4 \beta_{3}\right)\left(S_{-}+S_{-k}^{k}\right)\left(s_{\omega}^{(+1)}+s_{\omega l}^{(+1) l}\right) \delta_{i j}+ \\
& -2\left(\rho_{3}+4 \beta_{3}\right) S_{-i j}\left(s_{\omega}^{(+1)}+s_{\omega k}^{(+1) k}\right)-2\left(\rho_{3}+4 \beta_{3}\right) s_{\omega i j}^{(+1)}\left(S_{-}+S_{-k}^{k}\right) \\
& +\left(\rho_{3}+4 \beta_{3}\right)\left(S_{-}+S_{-k}^{k}\right)\left(s_{\omega}^{(+2)}+s_{\omega l}^{(+2) l}\right) \delta_{i j} \\
& -2\left(\rho_{3}+4 \beta_{3}\right) S_{-i j}\left(s^{(+2)}+s_{\omega k}^{(+1) k}\right)-2\left(\rho_{3}+4 \beta_{3}\right) s_{\omega i j}^{(+2)}\left(S_{-}+S_{-k}^{k}\right)
\end{aligned}
$$


For $\mathcal{L}_{I}^{4 A}$ :

$$
\begin{aligned}
& T_{I}^{4 A}{ }_{i j}=2\left(\left|\vec{e}^{[12]}\right|^{2}+\left|\vec{b}^{[12]}\right|^{2}\right) \delta_{i j}-4\left(\vec{e}_{i}^{[12]} \vec{e}_{j}^{[12]}+\vec{b}_{i}^{[12]} \vec{b}_{j}^{[12]}\right)+ \\
& +2\left(\vec{e}^{[12]} \cdot \vec{e}^{[21]}+\vec{b}^{[12]} \cdot \vec{b}^{[21]}\right) \delta_{i j}-4\left(\vec{e}_{i}^{[12]} \vec{e}_{j}^{[21]}+\vec{e}_{i}^{[21]} \vec{e}_{j}^{[12]}+\vec{b}_{i}^{[12]} \vec{b}_{j}^{[21]}+\vec{b}_{i}^{[21]} \vec{b}_{j}^{[12]}\right)+ \\
& +4\left(\vec{e}^{[13+]} \cdot \vec{e}^{[13-]}+\vec{b}^{[13+]} \cdot \vec{b}^{[13-]}\right) \delta_{i j}-8\left(\vec{e}_{i}^{[13+]} \vec{e}_{j}^{[13-]}+\vec{e}_{i}^{[13-]} \vec{e}_{j}^{[13+]}+\vec{b}_{i}^{[13+]} \vec{b}_{j}^{[13-]}+\vec{b}_{i}^{[13-]} \vec{b}_{j}^{[13+]}\right)+ \\
& +4\left(\vec{e}^{[23+]} \cdot \vec{e}^{[23-]}+\vec{b}^{[23+]} \cdot \vec{b}^{[23-]}\right) \delta_{i j}-8\left(\vec{e}_{i}^{[23+]} \vec{e}_{j}^{[23-]}+\vec{e}_{i}^{[23-]} \vec{e}_{j}^{[23+]}+\vec{b}_{i}^{[23+]} \vec{b}_{j}^{[23-]}+\vec{b}_{i}^{[23-]} \vec{b}_{j}^{[23+]}\right)+ \\
& +8\left(\vec{e}^{[13+]} \cdot \vec{e}^{[23-]}+\vec{b}^{[23-]} \cdot \vec{b}^{[13+]}\right) \delta_{i j}-16\left(\vec{e}_{i}^{[13+]} \vec{e}_{j}^{[23-]}+\vec{e}_{i}^{[23-]} \vec{e}_{j}^{[13+]}+\vec{b}_{i}^{[13+]} \vec{b}_{j}^{[23-]}+\vec{b}_{i}^{[23-]} \vec{b}_{j}^{[13+]}\right)+ \\
& +4\left(\vec{e}^{[12]} \cdot \vec{e}^{[+-]}+\vec{b}^{[12]} \cdot \vec{b}^{[+-]}\right) \delta_{i j}-8\left(\vec{e}_{i}^{[12]} \vec{e}_{j}^{[+-]}+\vec{e}_{i}^{[+-]} \vec{e}_{j}^{[12]}+\vec{b}_{i}^{[12]} \vec{b}_{j}^{[+-]}+\vec{b}_{i}^{[+-]} \vec{b}_{j}^{[12]}\right) \\
& -4\left(\vec{e}^{(12)} \cdot \vec{e}_{\omega}^{(12)}+\vec{b}^{(12)} \cdot \vec{b}_{\omega}^{(12)}\right) \delta_{i j}+16\left(\vec{e}_{i}^{(12)} \vec{e}_{\omega j}^{(12)}+\vec{e}_{\omega i}^{(12)} \vec{e}_{j}^{(12)}+\vec{b}_{i}^{(12)} \vec{b}_{\omega j}^{(12)}+\vec{b}_{\omega i}^{(12)} \vec{b}_{j}^{(12)}\right)+ \\
& -8\left(\left|\vec{e}_{\omega}^{(12)}\right|^{2}+\left|\vec{b}_{\omega}^{[12]}\right|^{2}\right) \delta_{i j}+16\left(\vec{e}_{\omega i}^{(12)} \vec{e}_{\omega j}^{(12)}+\vec{b}_{\omega i}^{(12)} \vec{b}_{\omega j}^{(12)}\right)+ \\
& +2\left(\vec{e}^{(13+)} \cdot \vec{e}^{(13-)}+\vec{b}^{(13+)} \cdot \vec{b}^{(13-)}\right) \delta_{i j}-8\left(\vec{e}_{i}^{(13+)} \vec{e}_{j}^{(13-)}+\vec{e}_{i}^{(13-)} \vec{e}_{j}^{(13+)}+\right. \\
& \left.+\vec{b}_{i}^{(13+)} \vec{b}_{j}^{(13-)}+\vec{b}_{i}^{(13-)} \vec{b}_{j}^{(13-)}\right)+ \\
& +2\left(\vec{e}^{(13+)} \cdot \vec{e}^{(13-)}+\vec{b}^{(13+)} \cdot \vec{b}^{(13-)}\right) \delta_{i j}-8\left(\vec{e}_{i}^{(13+)} \vec{e}_{j}^{(13-)}+\vec{e}_{i}^{(13-)} \vec{e}_{j}^{(13+)}+\right. \\
& \left.+\vec{b}_{i}^{(13+)} \vec{b}_{j}^{(13-)}+\vec{b}_{i}^{(13-)} \vec{b}_{j}^{(13+)}\right)+ \\
& +2\left(\vec{e}^{(23+)} \cdot \vec{e}^{(23-)}+\vec{b}^{(23+)} \cdot \vec{b}^{(23-)}\right) \delta_{i j}-8\left(\vec{e}_{i}^{(23+)} \vec{e}_{j}^{(23-)}+\vec{e}_{i}^{(23-)} \vec{e}_{j}^{(23+)}+\right. \\
& \left.+\vec{b}_{i}^{(23+)} \vec{b}_{j}^{(23-)}+\vec{b}_{i}^{(23-)} \vec{b}_{j}^{(23+)}\right)+ \\
& +4\left(\vec{e}^{(13+)} \cdot \vec{e}^{(23-)}+\vec{b}^{(13+)} \cdot \vec{b}^{(23-)}\right) \delta_{i j}-16\left(\vec{e}_{i}^{(13+)} \vec{e}_{j}^{(23-)}+\vec{e}_{i}^{(23-)} \vec{e}_{j}^{(13+)}+\right. \\
& \left.+\vec{b}_{i}^{(13+)} \vec{b}_{j}^{(23-)}+\vec{b}_{i}^{(23-)} \vec{b}_{j}^{(13+)}\right)+ \\
& -2 i\left(\vec{e}^{(13+)} \cdot \vec{e}_{\omega}^{(24-)}+\vec{b}^{(13+)} \cdot \vec{b}_{\omega}^{(24-)}\right) \delta_{i j}+8 i\left(\vec{e}_{i}^{(13+)} \vec{e}_{\omega j}^{(24-)}+\vec{e}_{\omega i}^{(24-)} \vec{e}_{j}^{(13+)}+\right. \\
& \left.+\vec{b}_{i}^{(13+)} \vec{b}_{\omega j}^{(24-)}+\vec{b}_{\omega i}^{(24-)} \vec{b}_{j}^{(13+)}\right)+ \\
& +2 i\left(\vec{e}^{(13-)} \cdot \vec{e}_{\omega}^{(24+)}+\vec{b}^{(13-)} \cdot \vec{b}_{\omega}^{(24+)}\right) \delta_{i j}-8 i\left(\vec{e}_{i}^{(13-)} \vec{e}_{\omega j}^{(24+)}+\vec{e}_{\omega i}^{(24+)} \vec{e}_{j}^{(13-)}+\right. \\
& \left.+\vec{b}_{i}^{(13-)} \vec{b}_{\omega j}^{(24+)}+\vec{b}_{\omega i}^{(24+)} \vec{b}_{j}^{(13-)}\right)+ \\
& -2 i\left(\vec{e}^{(23+)} \cdot \vec{e}_{\omega}^{(14-)}+\vec{b}^{(23+)} \cdot \vec{b}_{\omega}^{(14-)}\right) \delta_{i j}+8 i\left(\vec{e}_{i}^{(23+)} \vec{e}_{\omega j}^{(14-)}+\vec{e}_{\omega i}^{(14-)} \vec{e}_{j}^{(23+)}+\right. \\
& \left.+\vec{b}_{i}^{(23+)} \vec{b}_{\omega j}^{(14-)}+\vec{b}_{\omega i}^{(14-)} \vec{b}_{j}^{(23+)}\right)+ \\
& +2 i\left(\vec{e}^{(23-)} \cdot \vec{e}_{\omega}^{(14+)}+\vec{b}^{(23-)} \cdot \vec{b}_{\omega}^{(14+)}\right) \delta_{i j}-8 i\left(\vec{e}_{i}^{(23-)} \vec{e}_{\omega j}^{(14+)}+\vec{e}_{\omega i}^{(14+)} \vec{e}_{j}^{(23-)}+\right. \\
& \left.+\vec{b}_{i}^{(23-)} \vec{b}_{\omega j}^{(14+)}+\vec{b}_{\omega i}^{(14+)} \vec{b}_{j}^{(23-)}\right)+ \\
& -8 i\left(\vec{e}_{\omega}^{(13+)} \cdot \vec{e}_{\omega}^{(24-)}+\vec{b}_{\omega}^{(13+)} \cdot \vec{b}_{\omega}^{(24-)}\right) \delta_{i j}+32 i\left(\vec{e}_{\omega i}^{(13+)} \vec{e}_{\omega j}^{(24-)}+\vec{e}_{\omega i}^{(24-)} \vec{e}_{\omega j}^{(13+)}+\right. \\
& \left.+\vec{b}_{\omega i}^{(13+)} \vec{b}_{\omega j}^{(24-)}+\vec{b}_{\omega i}^{(24-)} \vec{b}_{\omega j}^{(13+)}\right)+ \\
& +8 i\left(\vec{e}_{\omega}^{(13-)} \cdot \vec{e}_{\omega}^{(24+)}+\vec{b}_{\omega}^{(13-)} \cdot \vec{b}_{\omega}^{(24+)}\right) \delta_{i j}-32 i\left(\vec{e}_{\omega i}^{(13-)} \vec{e}_{\omega j}^{(24+)}+\vec{e}_{\omega i}^{(24+)} \vec{e}_{\omega j}^{(13-)}+\right. \\
& \left.+\vec{b}_{\omega i}^{(13-)} \vec{b}_{\omega j}^{(24+)}+\vec{b}_{\omega i}^{(24+)} \vec{b}_{\omega j}^{(13-)}\right)+
\end{aligned}
$$




$$
\begin{aligned}
& -8 i\left(\vec{e}_{\omega}^{(14-)} \cdot \vec{e}_{\omega}^{(23+)}+\vec{b}_{\omega}^{(14-)} \cdot \vec{b}_{\omega}^{(23+)}\right) \delta_{i j}+32 i\left(\vec{e}_{\omega i}^{(14-)} \vec{e}_{\omega j}^{(23+)}+\vec{e}_{\omega i}^{(23+)} \vec{e}_{\omega j}^{(14-)}+\right. \\
& \left.+\vec{b}_{\omega i}^{(14-)} \vec{b}_{\omega j}^{(23+)}+\vec{b}_{\omega i}^{(23+)} \vec{b}_{\omega j}^{(14-)}\right)+ \\
& +8 i\left(\vec{e}_{\omega}^{(14+)} \cdot \vec{e}_{\omega}^{(23-)}+\vec{b}_{\omega}^{(14+)} \cdot \vec{b}_{\omega}^{(23-)}\right) \delta_{i j}-32 i\left(\vec{e}_{\omega i}^{(14+)} \vec{e}_{\omega j}^{(23-)}+\vec{e}_{\omega i}^{(23-)} \vec{e}_{\omega j}^{(14+)}+\right. \\
& \left.+\vec{b}_{\omega i}^{(14+)} \vec{b}_{\omega j}^{(23-)}+\vec{b}_{\omega i}^{(23-)} \vec{b}_{\omega j}^{(14+)}\right)+ \\
& -i\left(\vec{e}^{(13-)} \cdot \vec{e}^{(24+)}+\vec{b}^{(13-)} \cdot \vec{b}^{(24+)}\right) \delta_{i j}+4 i\left(\vec{e}_{i}^{(13-)} \vec{e}_{j}^{(24+)}+\vec{e}_{i}^{(24+)} \vec{e}_{j}^{(13-)}+\right. \\
& \left.+\vec{b}_{i}^{(13-)} \vec{b}_{j}^{(24+)}+\vec{b}_{i}^{(24+)} \vec{b}_{j}^{(13-)}\right)+ \\
& +i\left(\vec{e}^{(13+)} \cdot \vec{e}^{(24-)}+\vec{b}^{(13+)} \cdot \vec{b}^{(24-)}\right) \delta_{i j}-4 i\left(\vec{e}_{i}^{(13+)} \vec{e}_{j}^{(24-)}+\vec{e}_{i}^{(24-)} \vec{e}_{j}^{(13+)}+\right. \\
& \left.+\vec{b}_{i}^{(13+)} \vec{b}_{j}^{(24-)}+\vec{b}_{i}^{(24-)} \vec{b}_{j}^{(13+)}\right)+ \\
& -i\left(\vec{e}^{(14+)} \cdot \vec{e}^{(23-)}+\vec{b}^{(14+)} \cdot \vec{b}^{(23-)}\right) \delta_{i j}+4 i\left(\vec{e}_{i}^{(14+)} \vec{e}_{j}^{(23-)}+\vec{e}_{i}^{(23-)} \vec{e}_{j}^{(14+)}+\right. \\
& \left.+\vec{b}_{i}^{(14+)} \vec{b}_{j}^{(23-)}+\vec{b}_{i}^{(23-)} \vec{b}_{j}^{(14+)}\right)+ \\
& +i\left(\vec{e}^{(14-)} \cdot \vec{e}^{(23+)}+\vec{b}^{(14-)} \cdot \vec{b}^{(23+)}\right) \delta_{i j}-4 i\left(\vec{e}_{i}^{(14-)} \vec{e}_{j}^{(23+)}+\vec{e}_{i}^{(23+)} \vec{e}_{j}^{(14-)}+\right. \\
& \left.+\vec{b}_{i}^{(14-)} \vec{b}_{j}^{(23+)}+\vec{b}_{i}^{(23+)} \vec{b}_{j}^{(14-)}\right)+ \\
& -2 i\left(\vec{e}^{(14-)} \cdot \vec{e}_{\omega}^{(23+)}+\vec{b}^{(14-)} \cdot \vec{b}_{\omega}^{(23+)}\right) \delta_{i j}+8 i\left(\vec{e}_{i}^{(14-)} \vec{e}_{\omega j}^{(23+)}+\vec{e}_{\omega i}^{(23+)} \vec{e}_{j}^{(14-)}+\right. \\
& \left.+\vec{b}_{i}^{(14-)} \vec{b}_{\omega j}^{(23+)}+\vec{b}_{\omega i}^{(23+)} \vec{b}_{j}^{(14-)}\right)+ \\
& +2 i\left(\vec{e}^{(14+)} \cdot \vec{e}_{\omega}^{(23-)}+\vec{b}^{(14+)} \cdot \vec{b}_{\omega}^{(23-)}\right) \delta_{i j}-8 i\left(\vec{e}_{i}^{(14+)} \vec{e}_{\omega j}^{(23-)}+\vec{e}_{\omega i}^{(23-)} \vec{e}_{j}^{(14+)}+\right. \\
& \left.+\vec{b}_{i}^{(14+)} \vec{b}_{\omega j}^{(23-)}+\vec{b}_{\omega i}^{(23-)} \vec{b}_{j}^{(14+)}\right)+ \\
& -2 i\left(\vec{e}^{(24-)} \cdot \vec{e}_{\omega}^{(13+)}+\vec{b}^{(24-)} \cdot \vec{b}_{\omega}^{(13+)}\right) \delta_{i j}+8 i\left(\vec{e}_{i}^{(24-)} \vec{e}_{\omega j}^{(13+)}+\vec{e}_{\omega i}^{(13+)} \vec{e}_{j}^{(24-)}+\right. \\
& \left.+\vec{b}_{i}^{(24-)} \vec{b}_{\omega j}^{(13+)}+\vec{b}_{\omega i}^{(13+)} \vec{b}_{j}^{(24-)}\right)+ \\
& +2 i\left(\vec{e}^{(24+)} \cdot \vec{e}_{\omega}^{(13-)}+\vec{b}^{(24+)} \cdot \vec{b}_{\omega}^{(13-)}\right) \delta_{i j}-8 i\left(\vec{e}_{i}^{(24+)} \vec{e}_{\omega j}^{(13-)}+\vec{e}_{\omega i}^{(13-)} \vec{e}_{j}^{(24+)}+\right. \\
& \left.+\vec{b}_{i}^{(24+)} \vec{b}_{\omega j}^{(13-)}+\vec{b}_{\omega i}^{(13-)} \vec{b}_{j}^{(24+)}\right)+ \\
& -8\left(\vec{e}^{(+-)} \cdot \vec{e}_{\omega}^{(+-)}+\vec{b}^{(+-)} \cdot \vec{b}_{\omega}^{(+-)}\right) \delta_{i j}-32\left(\vec{e}_{i}^{(+-)} \vec{e}_{\omega j}^{(+-)}+\vec{e}_{\omega i}^{(+-)} \vec{e}_{j}^{(+-)}+\right. \\
& \left.+\vec{b}_{i}^{(+-)} \vec{b}_{\omega j}^{(+-)}+\vec{b}_{\omega i}^{(+-)} \vec{b}_{j}^{(+-)}\right)+ \\
& -16\left(\vec{e}_{\omega}^{(+-)} \cdot \vec{e}_{\omega}^{(+-)}+\vec{b}_{\omega}^{(+-)} \cdot \vec{b}_{\omega}^{(+-)}\right) \delta_{i j}-64\left(\vec{e}_{\omega i}^{(+-)} \vec{e}_{\omega j}^{(+-)}+\vec{e}_{\omega i}^{(+-)} \vec{e}_{\omega j}^{(+-)}+\right. \\
& \left.+\vec{b}_{\omega i}^{(+-)} \vec{b}_{\omega j}^{(+-)}+\vec{b}_{\omega i}^{(+-)} \vec{b}_{\omega j}^{(+-)}\right)+
\end{aligned}
$$


For $\mathcal{L}_{I}^{4 S}$ :

$$
\begin{aligned}
& T_{I}^{4 S}{ }_{i j}=\left(s^{[12]}+s_{k}^{[12] k}\right)^{2} \delta_{i j}-4 s_{i j}^{[12]}\left(s^{[12]}+s_{k}^{[12] k}\right)+ \\
& +\left(s^{[12]}+s_{k+}^{[12] k}\right)\left(s^{[21]}+s_{l+}^{[21] l}\right) \delta_{i j}-2 s_{i j}^{[12]}\left(s^{[21]}+s_{k-}^{[21] k}\right)-2 s_{i j}^{[21]}\left(s^{[12]}+s_{k-}^{[12] k}\right)+ \\
& +2\left(s^{[13+]}+s_{k+}^{[13+] k}\right)\left(s^{[13-]}+s_{l+}^{[13-] l}\right) \delta_{i j}-4 s_{i j}^{[13+]}\left(s^{[13-]}+s_{k-}^{[13-] k}\right)-4 s_{i j}^{[13-]}\left(s^{[13+]}+s_{k-}^{[13+] k}\right)+ \\
& +2\left(s^{[23+]}+s_{k+}^{[23+] k}\right)\left(s^{[23-]}+s_{l+}^{[23-] l}\right) \delta_{i j}-4 s_{i j}^{[23+]}\left(s^{[23-]}+s_{k-}^{[23-] k}\right)-4 s_{i j}^{[23-]}\left(s^{[23+]}+s_{k-}^{[23+] k}\right)+ \\
& +4\left(s^{[13+]}+s_{k+}^{[13+] k}\right)\left(s^{[23-]}+s_{l+}^{[23-] l}\right) \delta_{i j}-8 s_{i j}^{[13+]}\left(s^{[23-]}+s_{k-}^{[23-] k}\right)-8 s_{i j}^{[23-]}\left(s^{[13+]}+s_{k-}^{[13+] k}\right)+ \\
& +2\left(s^{[+-]}+s_{k}^{[+-] k}\right)^{2} \delta_{i j}-8 s_{i j}^{[+-]}\left(s^{[+-]}+s_{k}^{[+-] k}\right)+\left(s^{(11)}+s_{k}^{(11) k}\right)^{2} \delta_{i j}-4 s_{i j}^{(11)}\left(s^{(11)}+s_{k}^{(11) k}\right)+ \\
& +\left(s^{(11)}+s_{k}^{(11) k}\right)^{2} \delta_{i j}-4 s_{i j}^{(11)}\left(s^{(11)}+s_{k}^{(11) k}\right)+\left(s^{(22)}+s_{k}^{(22) k}\right)^{2} \delta_{i j}-4 s_{i j}^{(22)}\left(s^{(22)}+s_{k}^{(22) k}\right) \\
& +\left(s^{(11)}+s_{k}^{(11) k}\right)\left(s_{\omega}^{(11)}+s_{\omega l}^{(11) l}\right) \delta_{i j}-2 s_{i j}^{(11)}\left(s_{\omega}^{(11)}+s_{\omega k}^{(11) k}\right)-2 s_{\omega i j}^{(11)}\left(s^{(11)}+s_{k}^{(11) k}\right)+ \\
& +\left(s^{(22)}+s_{k}^{(22) k}\right)\left(s_{\omega}^{(22)}+s_{\omega l}^{(22) l}\right) \delta_{i j}-2 s_{i j}^{(22)}\left(s_{\omega}^{(22)}+s_{\omega k}^{(22) k}\right)-2 s_{\omega i j}^{(22)}\left(s^{(22)}+s_{k}^{(22) k}\right)+ \\
& +2\left(s_{\omega}^{(11)}+s_{\omega k}^{(11) k}\right)^{2} \delta_{i j}-8 s_{\omega i j}^{(11)}\left(s_{\omega}^{(11)}+s_{\omega k}^{(11) k}\right)+2\left(s_{\omega}^{(22)}+s_{\omega k}^{(22) k}\right)^{2} \delta_{i j}-8 s_{\omega i j}^{(22)}\left(s_{\omega}^{(22)}+s_{\omega k}^{(22) k}\right)+ \\
& +2\left(s^{(11)}+s_{k}^{(11) k}\right)\left(s^{(12)}+s_{l}^{(12) l}\right) \delta_{i j}-4 s_{i j}^{(11)}\left(s^{(12)}+s_{k}^{(12) k}\right)-4 s_{i j}^{(12)}\left(s^{(11)}+s_{k}^{(11) k}\right)+ \\
& +2\left(s^{(22)}+s_{k}^{(22) k}\right)\left(s^{(12)}+s_{l}^{(12) l}\right) \delta_{i j}-4 s_{i j}^{(22)}\left(s^{(12)}+s_{k}^{(12) k}\right)-4 s_{i j}^{(12)}\left(s^{(22)}+s_{k}^{(22) k}\right)+ \\
& +4\left(s^{(12)}+s_{k}^{(12) k}\right)\left(s_{\omega}^{(11)}+s_{\omega l}^{(11) l}\right) \delta_{i j}-8 s_{i j}^{(12)}\left(s_{\omega}^{(11)}+s_{\omega k}^{(11) k}\right)-8 s_{\omega i j}^{(11)}\left(s^{(12)}+s_{k}^{(12) k}\right)+ \\
& +4\left(s^{(12)}+s_{k}^{(12) k}\right)\left(s_{\omega}^{(22)}+s_{\omega l}^{(22) l}\right) \delta_{i j}-8 s_{i j}^{(12)}\left(s_{\omega}^{(22)}+s_{\omega k}^{(22) k}\right)-8 s_{\omega i j}^{(22)}\left(s^{(12)}+s_{k}^{(12) k}\right)+ \\
& +8\left(s_{\omega}^{(12)}+s_{\omega k}^{(12) k}\right)\left(s_{\omega}^{(11)}+s_{\omega l}^{(11) l}\right) \delta_{i j}-16 s_{\omega i j}^{(12)}\left(s_{\omega}^{(11)}+s_{\omega k}^{(11) k}\right)-16 s_{\omega i j}^{(11)}\left(s^{(12)}+s_{\omega k}^{(12) k}\right)+ \\
& +8\left(s_{\omega}^{(12)}+s_{\omega k}^{(12) k}\right)\left(s_{\omega}^{(22)}+s_{\omega l}^{(22) l}\right) \delta_{i j}-16 s_{\omega i j}^{(12)}\left(s_{\omega}^{(22)}+s_{\omega k}^{(22) k}\right)-16 s_{\omega i j}^{(22)}\left(s_{\omega}^{(12)}+s_{\omega k}^{(12) k}\right)+ \\
& +2\left(s^{(11)}+s_{k}^{(11) k}\right)\left(s^{(22)}+s_{l}^{(22) l}\right) \delta_{i j}-4 s_{i j}^{(11)}\left(s^{(22)}+s_{k}^{(22) k}\right)-4 s_{i j}^{(22)}\left(s^{(22)}+s_{k}^{(22) k}\right)+ \\
& +2\left(s^{(11)}+s_{k}^{(11) k}\right)\left(s^{+-3}+s_{l}^{+-3 l}\right) \delta_{i j}-4 s_{i j}^{(11)}\left(s^{+-3}+s_{k}^{+-3 k}\right)-4 s_{i j}^{+-3}\left(s^{(11)}+s_{k}^{(11) k}\right)+ \\
& +2\left(s^{(22)}+s_{k}^{(22) k}\right)\left(s^{+-3}+s_{l}^{+-3 l}\right) \delta_{i j}-4 s_{i j}^{(22)}\left(s^{+-3}+s_{k}^{+-3 k}\right)-4 s_{i j}^{+-3}\left(s^{(22)}+s_{k}^{(22) k}\right)+ \\
& +4\left(s^{(12)}+s_{k}^{(12) k}\right)^{2} \delta_{i j}-8 s_{i j}^{(12)}\left(s^{(12)}+s_{k}^{(12) k}\right)+8\left(s_{\omega}^{(12)}+s_{\omega k}^{(12) k}\right)^{2} \delta_{i j}-16 s_{\omega i j}^{(12)}\left(s_{\omega}^{(12)}+s_{\omega k}^{(12) k}\right)+ \\
& +4\left(s^{(12)}+s_{k}^{(12) k}\right)\left(s_{\omega}^{(12)}+s_{\omega l}^{(12) l}\right) \delta_{i j}-8 s_{\omega i j}^{(12)}\left(s^{(12)}+s_{k}^{(12) k}\right)-8 s_{i j}^{(12)}\left(s_{\omega}^{(12)}+s_{\omega k}^{(12) k}\right)+
\end{aligned}
$$




$$
\begin{aligned}
& +2\left(s^{(13+)}+s_{k}^{(13+) k}\right)\left(s^{(13-)}+s_{l}^{(13-) l}\right) \delta_{i j}-4 s_{i j}^{(13+)}\left(s^{(13-)}+s_{k}^{(13-) k}\right)-4 s_{i j}^{(13-)}\left(s^{(13+)}+s_{k}^{(13+) k}\right)+ \\
& +2\left(s^{(23+)}+s_{k}^{(23+) k}\right)\left(s^{(23-)}+s_{l}^{(23-) l}\right) \delta_{i j}-4 s_{i j}^{(23+)}\left(s^{(23-)}+s_{k}^{(23-) k}\right)-4 s_{i j}^{(23-)}\left(s^{(23+)}+s_{k}^{(23+) k}\right)+ \\
& +4\left(s^{(13+)}+s_{k}^{(13+) k}\right)\left(s^{(23-)}+s_{l}^{(23-) l}\right) \delta_{i j}-8 s_{i j}^{(13+)}\left(s^{(23-)}+s_{k}^{(23-) k}\right)-8 s_{i j}^{(23-)}\left(s^{(13+)}+s_{k}^{(13+) k}\right)+ \\
& +4\left(s^{(12)}+s_{k}^{(12) k}\right)\left(s^{+-3}+s_{l}^{+-3 l}\right) \delta_{i j}-8 s_{i j}^{(12)}\left(s^{+-3}+s_{k}^{+-3 k}\right)-8 s_{i j}^{+-3}\left(s^{(12)}+s_{k}^{(12) k}\right)+ \\
& +4\left(s^{(12)}+s_{k}^{(12) k}\right)\left(s^{+-3}+s_{l}^{+-3 l}\right) \delta_{i j}-8 s_{i j}^{(12)}\left(s^{+-3}+s_{k}^{+-3 k}\right)-8 s_{i j}^{+-3}\left(s^{(12)}+s_{k}^{(12) k}\right)+ \\
& +2\left(s^{+-3}+s_{k}^{+-3 k}\right)\left(s^{+-4}+s_{l}^{+-4 l}\right) \delta_{i j}-4 s_{i j}^{+-3}\left(s^{+-4}+s_{k}^{+-4 k}\right)-4 s_{i j}^{+-4}\left(s^{+-3}+s_{k}^{+-3 k}\right)+ \\
& +2 i\left(s^{(13+)}+s_{k}^{(13+) k}\right)\left(s_{\omega}^{(24-)}+s_{\omega l}^{(24-) l}\right) \delta_{i j}+ \\
& -4 i s_{i j}^{(13+)}\left(s_{\omega}^{(24-)}+s_{\omega k}^{(24-) k}\right)-4 i s_{\omega i j}^{(24-)}\left(s^{(13+)}+s_{k}^{(13+) k}\right)+ \\
& -2 i\left(s^{(13-)}+s_{k}^{(13-) k}\right)\left(s_{\omega}^{(24+)}+s_{\omega l}^{(24+) l}\right) \delta_{i j}+ \\
& +4 i s_{i j}^{(13-)}\left(s_{\omega}^{(24+)}+s_{\omega k}^{(24+) k}\right)+4 i s_{\omega i j}^{(24+)}\left(s^{(13-)}+s_{k}^{(13-) k}\right)+ \\
& +2 i\left(s^{(23+)}+s_{k}^{(23+) k}\right)\left(s_{\omega}^{(14-)}+s_{\omega l}^{(14-) l}\right) \delta_{i j} \\
& -4 i s_{i j}^{(23+)}\left(s_{\omega}^{(14-)}+s_{\omega k}^{(14-) k}\right)-4 i s_{\omega i j}^{(14-)}\left(s^{(23+)}+s_{k}^{(23+) k}\right)+ \\
& -2 i\left(s^{(23-)}+s_{k}^{(23-) k}\right)\left(s_{\omega}^{(14+)}+s_{\omega l}^{(14+) l}\right) \delta_{i j}+ \\
& +4 i s_{i j}^{(23-)}\left(s_{\omega}^{(14+)}+s_{\omega k}^{(14+) k}\right)+4 i s_{\omega i j}^{(14+)}\left(s^{(23-)}+s_{k}^{(23-) k}\right)+ \\
& +8 i\left(s_{\omega}^{(23+)}+s_{\omega k}^{(23+) k}\right)\left(s_{\omega}^{(24-)}+s_{\omega l}^{(24-) l}\right) \delta_{i j}+ \\
& -16 i s_{\omega i j}^{(23+)}\left(s_{\omega}^{(24-)}+s_{\omega k}^{(24-) k}\right)-16 i s_{\omega i j}^{(14-)}\left(s_{\omega}^{(23+)}+s_{\omega k}^{(23+) k}\right)+ \\
& -8 i\left(s_{\omega}^{(23-)}+s_{\omega k}^{(23-) k}\right)\left(s_{\omega}^{(14+)}+s_{\omega l}^{(14+) l}\right) \delta_{i j}+ \\
& +16 i s_{\omega i j}^{(23-)}\left(s_{\omega}^{(14+)}+s_{\omega k}^{(14+) k}\right)+16 i s_{\omega i j}^{(14+)}\left(s_{\omega}^{(23-)}+s_{\omega k}^{(23-) k}\right)+ \\
& +i\left(s^{(14+)}+s_{k}^{(14+) k}\right)\left(s^{(23-)}+s_{l}^{(23-) l}\right) \delta_{i j}+ \\
& -2 i s_{i j}^{(14+)}\left(s^{(23-)}+s_{k}^{(23-) k}\right)-2 i s_{i j}^{(23-)}\left(s^{(14+)}+s_{k}^{(14+) k}\right)+ \\
& -i\left(s^{(14-)}+s_{k}^{(14-) k}\right)\left(s_{\omega}^{(23+)}+s_{\omega l}^{(23+) l}\right) \delta_{i j}+ \\
& \\
& +13
\end{aligned}
$$




$$
\begin{aligned}
& +2 i s_{i j}^{(14-)}\left(s_{\omega}^{(23+)}+s_{\omega k}^{(23+) k}\right)+2 i s_{\omega i j}^{(23+)}\left(s^{(14-)}+s_{k}^{(14-) k}\right)+ \\
& +8 i\left(s_{\omega}^{(14-)}+s_{\omega k}^{(14-) k}\right)\left(s_{\omega}^{(23+)}+s_{\omega l}^{(23+) l}\right) \delta_{i j}+ \\
& -16 i s_{\omega i j}^{(14-)}\left(s_{\omega}^{(23+)}+s_{\omega k}^{(23+) k}\right)-16 i s_{\omega i j}^{(23+)}\left(s_{\omega}^{(14-)}+s_{\omega k}^{(14-) k}\right)+ \\
& -8 i\left(s_{\omega}^{(14+)}+s_{\omega k}^{(14+) k}\right)\left(s_{\omega}^{(23-)}+s_{\omega l}^{(23-) l}\right) \delta_{i j}+ \\
& +16 i s_{\omega i j}^{(23-)}\left(s_{\omega}^{(14+)}+s_{\omega k}^{(14+) k}\right)+16 i s_{\omega i j}^{(14+)}\left(s_{\omega}^{(23-)}+s_{\omega k}^{(23-) k}\right)+ \\
& +i\left(s^{(13-)}+s_{k}^{(13-) k}\right)\left(s^{(24+)}+s_{l}^{(24+) l}\right) \delta_{i j}+ \\
& -2 i s_{i j}^{(13-)}\left(s^{(24+)}+s_{k}^{(24+) k}\right)-2 i s_{i j}^{(24+)}\left(s^{(13-)}+s_{k}^{(13-) k}\right)+ \\
& -i\left(s^{(13+)}+s_{k}^{(13+) k}\right)\left(s^{(24-)}+s_{l}^{(24-) l}\right) \delta_{i j}+ \\
& +2 i s_{i j}^{(13+)}\left(s^{(24-)}+s_{k}^{(24-) k}\right)+2 i s_{i j}^{(24-)}\left(s^{(13+)}+s_{k}^{(13+) k}\right)+ \\
& +2 i\left(s_{\omega}^{(23+)}+s_{\omega k}^{(23+) k}\right)\left(s^{(14-)}+s_{l}^{(14-) l}\right) \delta_{i j}+ \\
& -4 i s_{\omega i j}^{(23+)}\left(s^{(14-)}+s_{k}^{(14-) k}\right)-4 i s_{i j}^{(14-)}\left(s_{\omega}^{(23+)}+s_{\omega k}^{(23+) k}\right)+ \\
& -2 i\left(s_{\omega}^{(23-)}+s_{\omega k}^{(23-) k}\right)\left(s^{(14+)}+s_{l}^{(14+) l}\right) \delta_{i j}+ \\
& +4 i s_{\omega i j}^{(23-)}\left(s^{(14+)}+s_{\omega k}^{(14+) k}\right)+4 i s_{i j}^{(14-)}\left(s_{\omega}^{(23-)}+s_{\omega k}^{(23-) k}\right)+
\end{aligned}
$$




$$
\begin{aligned}
& +2 i\left(s_{\omega}^{(13+)}+s_{\omega k}^{(13+) k}\right)\left(s^{(24-)}+s_{l}^{(24-) l}\right) \delta_{i j}+ \\
& -4 i s_{\omega i j}^{(13+)}\left(s^{(24-)}+s_{k}^{(24-) k}\right)-4 i s_{i j}^{(24-)}\left(s_{\omega}^{(13+)}+s_{\omega k}^{(13+) k}\right)+ \\
& -2 i\left(s_{\omega}^{(13-)}+s_{\omega k}^{(13-) k}\right)\left(s^{(24+)}+s_{l}^{(24+) l}\right) \delta_{i j}+ \\
& +4 i s_{\omega i j}^{(13-)}\left(s^{(24+)}+s_{\omega k}^{(24+) k}\right)+4 i s_{i j}^{(24+)}\left(s_{\omega}^{(13-)}+s_{\omega k}^{(13-) k}\right)+ \\
& -2\left(s^{[13+]}+s_{k}^{[13+] k}\right)\left(s^{[13-]}+s_{k}^{[13-] k}\right) \delta_{i j}+4 s_{i j}^{[13+]}\left(s^{[13-]}+s_{k}^{[13-] k}\right)+4 s_{i j}^{[13-]}\left(s^{[13+]}+s_{k}^{[13+] k}\right)+ \\
& -2\left(s^{[23+]}+s_{k}^{[23+] k}\right)\left(s^{[23-]}+s_{k}^{[23-] k}\right) \delta_{i j}+4 s_{i j}^{[23+]}\left(s^{[23-]}+s_{k}^{[23-] k}\right)+4 s_{i j}^{[23-]}\left(s^{[23+]}+s_{k}^{[23+] k}\right)+ \\
& -2\left(s^{[+-]}+s_{k}^{[+-] k}\right)^{2} \delta_{i j}+8 s_{i j}^{[+-]}\left(s^{[+-]}+s_{k}^{[+-] k}\right)+ \\
& -2\left(s^{[13+]}+s_{k}^{[13+] k}\right)\left(s^{[23-]}+s_{k}^{[23-] k}\right) \delta_{i j}+4 s_{i j}^{[13+]}\left(s^{[23-]}+s_{k}^{[23-] k}\right)+4 s_{i j}^{[23-]}\left(s^{[13+]}+s_{k}^{[13+] k}\right)+ \\
& +2\left(s^{[13-]}+s_{k}^{[13-] k}\right)\left(s^{[23+]}+s_{k}^{[23+] k}\right) \delta_{i j}-4 s_{i j}^{[13-]}\left(s^{[23+]}+s_{k}^{[23+] k}\right)-4 s_{i j}^{[23+]}\left(s^{[13-]}+s_{k}^{[13-] k}\right)+ \\
& +2 i\left(s^{[13-]}+s_{k}^{[13-] k}\right)\left(s^{[24+]}+s_{k}^{[24+] k}\right) \delta_{i j}-4 i s_{i j}^{[13-]}\left(s^{[24+]}+s_{k}^{[24+] k}\right)-4 i s_{i j}^{[24+]}\left(s^{[13-]}+s_{k}^{[13-] k}\right)+ \\
& -2 i\left(s^{[13+]}+s_{k}^{[13+] k}\right)\left(s^{[24-]}+s_{k}^{[24-] k}\right) \delta_{i j}+4 i s_{i j}^{[13+]}\left(s^{[24-]}+s_{k}^{[24-] k}\right)+4 i s_{i j}^{[24-]}\left(s^{[13+]}+s_{k}^{[13+] k}\right)+ \\
& +2\left(s^{(11)}+s_{k}^{(11) k}\right)\left(s_{\omega}^{(22)}+s_{\omega k}^{(22) k}\right) \delta_{i j}-4 s_{i j}^{(11)}\left(s_{\omega}^{(22)}+s_{\omega k}^{(22) k}\right)-4 s_{\omega i j}^{(22)}\left(s^{(11)}+s_{k}^{(11) k}\right)+ \\
& +4\left(s_{\omega}^{(11)}+s_{\omega k}^{(11) k}\right)\left(s_{\omega}^{(22)}+s_{\omega k}^{(22) k}\right) \delta_{i j}-8 s_{\omega i j}^{(11)}\left(s_{\omega}^{(22)}+s_{\omega k}^{(22) k}\right)-8 s_{\omega i j}^{(22)}\left(s_{\omega}^{(11)}+s_{\omega k}^{(11) k}\right)+ \\
& +2\left(s^{(13+)}+s_{k}^{(13+) k}\right)\left(s^{(13-)}+s_{k}^{(13-) k}\right) \delta_{i j}-4 s_{i j}^{(13+)}\left(s^{(13-)}+s_{k}^{(13-) k}\right)-4 s_{i j}^{(13-)}\left(s^{(13+)}+s_{k}^{(13+) k}\right)+ \\
& +2\left(s^{(23+)}+s_{k}^{(23+) k}\right)\left(s^{(23-)}+s_{k}^{(23-) k}\right) \delta_{i j}-4 s_{i j}^{(23+)}\left(s^{(23-)}+s_{k}^{(23-) k}\right)-4 s_{i j}^{(23-)}\left(s^{(23+)}+s_{k}^{(23+) k}\right)+ \\
& +8\left(s^{(13+)}+s_{k}^{(13+) k}\right)\left(s_{\omega}^{(13-)}+s_{\omega k}^{(13-) k}\right) \delta_{i j}+ \\
& -16 s_{i j}^{(13+)}\left(s_{\omega}^{(13-)}+s_{\omega k}^{(13-) k}\right)-16 s_{\omega i j}^{(13-)}\left(s^{(13+)}+s_{k}^{(13+) k}\right)+ \\
& +8\left(s^{(23+)}+s_{k}^{(23+) k}\right)\left(s_{\omega}^{(23-)}+s_{\omega k}^{(23-) k}\right) \delta_{i j}+ \\
& -16 s_{i j}^{(23+)}\left(s_{\omega}^{(23-)}+s_{\omega k}^{(23-) k}\right)-16 s_{\omega i j}^{(23-)}\left(s^{(23+)}+s_{k}^{(23+) k}\right)+ \\
& +16\left(s_{\omega}^{(13+)}+s_{\omega k}^{(13+) k}\right)\left(s_{\omega}^{(13-)}+s_{\omega k}^{(13-) k}\right) \delta_{i j}+ \\
& -32 s_{\omega i j}^{(13+)}\left(s_{\omega}^{(13-)}+s_{\omega k}^{(13-) k}\right)-32 s_{\omega i j}^{(13-)}\left(s_{\omega}^{(13+)}+s_{\omega k}^{(13+) k}\right)+ \\
& +16\left(s_{\omega}^{(23+)}+s_{\omega k}^{(23+) k}\right)\left(s_{\omega}^{(23-)}+s_{\omega k}^{(23-) k}\right) \delta_{i j}+ \\
& -32 s_{\omega i j}^{(23+)}\left(s_{\omega}^{(23-)}+s_{\omega k}^{(23-) k}\right)-32 s_{\omega i j}^{(23-)}\left(s_{\omega}^{(23+)}+s_{\omega k}^{(23+) k}\right)+ \\
& +4\left(s^{(13+)}+s_{k}^{(13+) k}\right)\left(s^{(23-)}+s_{k}^{(23-) k}\right) \delta_{i j}-4 s_{i j}^{(13+)}\left(s^{(23-)}+s_{k}^{(23-) k}\right)-4 s_{i j}^{(23-)}\left(s^{(13+)}+s_{k}^{(13+) k}\right)+
\end{aligned}
$$




$$
\begin{aligned}
& -4\left(s^{(13-)}+s_{k}^{(13-) k}\right)\left(s^{(23+)}+s_{k}^{(23+) k}\right) \delta_{i j}+4 s_{i j}^{(13-)}\left(s^{(23+)}+s_{k}^{(23+) k}\right)+4 s_{i j}^{(23+)}\left(s^{(13-)}+s_{k}^{(13-) k}\right)+ \\
& +8\left(s^{(13+)}+s_{k}^{(13+) k}\right)\left(s_{\omega}^{(13-)}+s_{\omega k}^{(13-) k}\right) \delta_{i j}-16 s_{i j}^{(13+)}\left(s_{\omega}^{(13-)}+s_{\omega k}^{(13-) k}\right)-16 s_{\omega i j}^{(13-)}\left(s^{(13+)}+s_{k}^{(13+) k}\right)+ \\
& -8\left(s^{(13-)}+s_{k}^{(13-) k}\right)\left(s_{\omega}^{(13+)}+s_{\omega k}^{(13+) k}\right) \delta_{i j}+16 s_{i j}^{(13-)}\left(s_{\omega}^{(13+)}+s_{\omega k}^{(13+) k}\right)-16 s_{\omega i j}^{(13+)}\left(s^{(13-)}+s_{k}^{(13-) k}\right)+ \\
& +16\left(s_{\omega}^{(13+)}+s_{\omega k}^{(13+) k}\right)\left(s_{\omega}^{(23-)}+s_{\omega k}^{(23-) k}\right) \delta_{i j}-32 s_{\omega i j}^{(13+)}\left(s_{\omega}^{(23-)}+s_{\omega k}^{(23-) k}\right)-32 s_{\omega i j}^{(23-)}\left(s_{\omega}^{(13+)}+s_{\omega k}^{(13+) k}\right)+ \\
& -16\left(s_{\omega}^{(13-)}+s_{\omega k}^{(13-) k}\right)\left(s_{\omega}^{(23+)}+s_{\omega k}^{(23+) k}\right) \delta_{i j}+32 s_{\omega i j}^{(13-)}\left(s_{\omega}^{(23+)}+s_{\omega k}^{(23+) k}\right)+32 s_{\omega i j}^{(23+)}\left(s_{\omega}^{(13-)}+s_{\omega k}^{(13) k}\right)+ \\
& -2\left(s^{(+-)}+s_{k}^{(+-) k}\right)^{2} \delta_{i j}+4 s_{i j}^{(+-)}\left(s^{(+-)}+s_{k}^{(+-) k}\right)+ \\
& +4\left(s^{+-3}+s_{k}^{+-3 k}\right)\left(s_{\omega}^{+-4}+s_{\omega k}^{+-4 k}\right) \delta_{i j}-8 s_{i j}^{+-3}\left(s_{\omega}^{+-4}+s_{\omega k}^{+-4 k}\right)-8 s_{\omega i j}^{+-4}\left(s^{+-3}+s_{k}^{+-3 k}\right)+ \\
& +8\left(s_{\omega}^{+-3}+s_{\omega k}^{+-3 k}\right)\left(s_{\omega}^{+-4}+s_{\omega k}^{+-4 k}\right) \delta_{i j}-16 s_{\omega i j}^{+-3}\left(s_{\omega}^{+-3}+s_{\omega k}^{+-4 k}\right)-16 s_{\omega i j}^{+-4}\left(s_{\omega}^{+-3}+s_{\omega k}^{+-3 k}\right)+ \\
& +4\left(s^{(11)}+s_{k}^{(11) k}\right)\left(s_{\omega}^{+-3}+s_{\omega k}^{+-3 k}\right) \delta_{i j}-8 s_{i j}^{(11)}\left(s_{\omega}^{+-3}+s_{\omega k}^{+-3 k}\right)-8 s_{\omega i j}^{+-3}\left(s^{(11)}+s_{k}^{(11) k}\right)+ \\
& +4\left(s^{(22)}+s_{k}^{(22) k}\right)\left(s_{\omega}^{+-3}+s_{\omega k}^{+-3 k}\right) \delta_{i j}-8 s_{i j}^{(22)}\left(s_{\omega}^{+-3}+s_{\omega k}^{+-3 k}\right)-8 s_{\omega i j}^{+-3}\left(s^{(22)}+s_{k}^{(22) k}\right)+ \\
& +8\left(s_{\omega}^{(11)}+s_{\omega k}^{(11) k}\right)\left(s_{\omega}^{+-3}+s_{\omega k}^{+-3 k}\right) \delta_{i j}-16 s_{\omega i j}^{(11)}\left(s_{\omega}^{+-3}+s_{\omega k}^{+-3 k}\right)-16 s_{\omega i j}^{+-3}\left(s_{\omega}^{(11)}+s_{\omega k}^{(11) k}\right)+ \\
& +8\left(s_{\omega}^{(22)}+s_{\omega k}^{(22) k}\right)\left(s_{\omega}^{+-3}+s_{\omega k}^{+-3 k}\right) \delta_{i j}-16 s_{\omega i j}^{(22)}\left(s_{\omega}^{+-3}+s_{\omega k}^{+-3 k}\right)-16 s_{\omega i j}^{+-3}\left(s_{\omega}^{(22)}+s_{\omega k}^{(22) k}\right)+ \\
& +8\left(s^{(12)}+s_{k}^{(12) k}\right)\left(s_{\omega}^{+-3}+s_{\omega k}^{+-3 k}\right) \delta_{i j}-16 s_{i j}^{(12)}\left(s_{\omega}^{+-3}+s_{\omega k}^{+-3 k}\right)-16 s_{\omega i j}^{+-3}\left(s^{(12)}+s_{k}^{(12) k}\right)+ \\
& +16\left(s_{\omega}^{(12)}+s_{\omega k}^{(12) k}\right)\left(s_{\omega}^{+-3}+s_{\omega k}^{+-3 k}\right) \delta_{i j}-32 s_{\omega i j}^{(12)}\left(s_{\omega}^{+-3}+s_{\omega k}^{+-3 k}\right)-32 s_{\omega i j}^{+-3}\left(s_{\omega}^{(12)}+s_{\omega k}^{(12) k}\right)
\end{aligned}
$$

\section{Noether Charges}

The charge transport through the four bosons interconnected quadruplet is made of by just one gauge parameter given by $\mathrm{U}(1) \times \mathrm{SO}(2)_{\text {global }}$. It yields the following three Noether identities [21],

$$
\begin{gathered}
\partial_{\mu} J_{N}^{\mu}=0 \quad \text { (symmetric charge conservation) } \\
\partial_{\nu} K^{\nu \mu}+J_{N}^{\mu}=0 \quad \text { (symmetric equation) }
\end{gathered}
$$

$$
K^{\mu \nu} \partial_{\mu} \partial_{\nu} \alpha=0 \quad \text { (symmetry constraint) }
$$

Eqs.(10.1) shows the electric charge conservation. Eqs.(10.2) and and (10.3) yield the relationship 


$$
\partial_{\mu} \partial_{\nu} K^{\nu \mu}=0
$$

where

$$
K^{\nu \mu}=k_{A} \frac{\partial \mathcal{L}}{\partial\left(\partial_{\nu} A_{\mu}\right)}+k_{U} \frac{\partial \mathcal{L}}{\partial\left(\partial_{\nu} U_{\mu}\right)}+k_{+} \frac{\partial \mathcal{L}}{\partial\left(\partial_{\nu} V_{\mu}^{+}\right)}+k_{-} \frac{\partial \mathcal{L}}{\partial\left(\partial_{\nu} V_{\mu}^{-}\right)}
$$

Taking $K^{\mu \nu}=K^{[\mu \nu]}+K^{(\mu \nu)}$ and $K_{(\mu \nu)}=\tilde{K}_{(\mu \nu)}+g_{\mu \nu} K_{\alpha}^{\alpha}$, onde derives the relationship

$$
\partial_{\mu} \partial_{\nu} \tilde{K}^{(\mu \nu)}+\square K_{\alpha}^{\alpha}=0
$$

which yields

$$
\sum_{I=1}^{4} k_{I} \square\left(S_{\alpha}^{\alpha I}+z_{\alpha}^{\alpha I}\right)=0
$$

Inserting eq.(10.7) in eq.(3.1), we have the systemic conservation law

$$
k_{I} \partial \cdot\left(m^{I}+l^{I}+c^{I}\right)=k_{I} \partial \cdot j^{I}
$$

where eq.(10.8) means that the systemic conservation law involving the fields set $\left\{A_{\mu}, U_{\mu}, V_{\mu}^{+}, V_{\mu}^{-}\right\}$.

\section{Angular Momentum}

The Lorentz Group systematizes the relativistic description of the spin, introduced by Uhlenbeck and Goudsmit as an exclusively quantum attribute. From Lorentz's rotation in space-time:

$$
x^{\prime \mu}=\Lambda_{\nu}^{\mu} x^{\nu}
$$

In the infinitesimal form:

$$
x^{\prime \mu}=\left(\delta^{\mu}{ }_{\nu}+\omega^{\mu}{ }_{\nu}\right) x^{\nu}+\mathcal{O}\left(\omega^{2}\right)
$$

or

$$
\delta x^{\prime \mu}=\omega^{\mu}{ }_{\nu} x^{\nu}+\mathcal{O}\left(\omega^{2}\right) \quad \text { onde } \quad \omega^{\mu}{ }_{\nu}=-\omega_{\nu}{ }^{\mu}
$$


The representations of $S O(1,3)$ are characterized by a pair of quantum numbers $(j, k)$. The transformation of the fields, $\phi_{i}$, which belong to this representation, is given by:

$$
\begin{aligned}
& \phi_{i}^{\prime}\left(x^{\prime}\right)=\exp { }^{\left[\frac{1}{2} \omega^{\alpha \beta} \Sigma_{\alpha \beta}\right]} \phi_{i}(x) \\
& \phi_{i}^{\prime}\left(x^{\prime}\right)=\phi_{i}(x)+\frac{1}{2} \omega^{\alpha \beta} \Sigma_{\alpha \beta} \phi_{i}(x)+\mathcal{O}\left(\omega^{2}\right)
\end{aligned}
$$

or,

$$
\delta_{\text {total }} \phi_{i}=\frac{1}{2} \omega^{\alpha \beta} \Sigma_{\alpha \beta} \phi_{i}(x)
$$

From the above expression and using $\delta_{\text {total }} f=\delta_{\text {func }} f+\delta x \partial_{\mu} f(x)$, we get

$$
\delta_{\text {func }} \phi_{i}=\frac{1}{2} \omega^{\alpha \beta} \Sigma_{\alpha \beta} \phi_{i}(x)-\omega^{\alpha}{ }_{\beta} x^{\beta} \partial_{\alpha} \phi_{i}(x)
$$

The conservation law associated with rotational invariance:

$$
\partial_{\mu}\left[\omega^{\alpha \beta} \delta^{\mu}{ }_{\alpha} x_{\beta} \mathcal{L}+\frac{\partial \mathcal{L}}{\partial \partial_{\mu} \phi_{i}}\left(\frac{1}{2} \omega^{\alpha \beta} \Sigma_{\alpha \beta} \phi_{i}(x)--\omega^{\alpha}{ }_{\beta} x^{\beta} \partial_{\alpha} \phi_{i}(x)\right)\right]=0
$$

Rewriting the above equation:

$$
\frac{1}{2} \omega^{\alpha \beta}\left[\left(\delta^{\mu}{ }_{\alpha} x_{\beta}-\delta^{\mu}{ }_{\beta} x_{\alpha}\right) \mathcal{L}+\frac{\partial \mathcal{L}}{\partial \partial_{\mu} \phi_{i}} \Sigma_{\alpha \beta} \phi_{i}(x)-\frac{\partial \mathcal{L}}{\partial \partial_{\mu} \phi_{i}}\left(x_{\beta} \partial_{\alpha} \phi_{i}(x)-x_{\alpha} \partial_{\beta} \phi_{i}(x)\right)\right]=0
$$

results

$$
\partial_{\mu}\left[\frac{\partial \mathcal{L}}{\partial \partial_{\mu} \phi_{i}} \Sigma_{\alpha \beta} \phi_{i}(x)+x_{\alpha}\left(\frac{\partial \mathcal{L}}{\partial \partial_{\mu} \phi_{i}} \partial_{\beta} \phi_{i}(x)-\delta^{\mu}{ }_{\beta} \mathcal{L}\right)-x_{\beta}\left(\frac{\partial \mathcal{L}}{\partial \partial_{\mu} \phi_{i}} \partial_{\alpha} \phi_{i}(x)-\delta^{\mu}{ }_{\alpha} \mathcal{L}\right)\right]=0
$$

We obtain the expression of conservation of angular momentum:

$$
\partial_{\mu}\left[x_{\alpha} T_{\beta}^{\mu}-x_{\beta} T_{\alpha}^{\mu}\right]=0
$$


The above expression leads us to the definition of:

1. The density of orbital angular momentum is:

$$
L_{\alpha \beta}^{\mu}=\left(x_{\alpha} T_{\beta}^{\mu}-x_{\beta} T_{\alpha}^{\mu}\right)
$$

2. The density of intrinsic angular momentum or spin current density

$$
S^{\mu}=\frac{\partial \mathcal{L}}{\partial \partial_{\mu} \phi_{i}} \Sigma_{\alpha \beta} \phi_{i}
$$

\section{Conclusion}

Three periods classify the electromagnetism knowledgement. Charges and currents, charges and fields, fields and fields. Currently the expectative is on understanding how fields work as their own sources. Understand how far there is an electromagnetism not depending on electric charge. A nonlinear EM physics just depending on fields.

At modern time electromagnetism started to be studied with the book De Magnete written by William Gilbert in 1600. Electrostatic machines were developed by Otto von Guericke in 1672. At 18 century different observations introduced electric charge. It was understood through condensates, discharges, bioelectricity, batteries by Pieter von Musschenbrock, Benjamin Franklin, Galvani, Du Fay, Volta and others. EM was understood as consequence from charged matter.

The second EM phase showed that nature is more than matter. Differently from $17^{\text {th }}$ century when Newton laws were edified in terms of matter, Faraday introduced at $19^{\text {th }}$ century the meaning of fields[24]. Physical behaviors become explained through lines of force. New variables in order to interpret physical laws. This led Maxwell to analyses the phenomena through charges and fields. Physical laws should no more be expressed just by matter. While at one side, Coulomb and Ampère laws depend directly on electric charge and currents; on the other hand, Faraday and Gauss magnetic laws not.

Under this complementary view, Maxwell incorporated the displacement current. Believed in the logic of identifying a current composed by electric field. Followed that, physical entities as EM energy, momentum, light propagation and so on, become expressed by fields. Nevertheless, Maxwell legacy is on fields constrained to electric charge. It was summarized by the H. Lorentz sentence written in 1895 at Philosophical Magazine: 'The electromagnetism axiom, is that electromagnetic field is that electromagnetic field is an excited state stablished in the space through the electric charge. Based on that the vector $\vec{E}$ and $\vec{B}$ build up on electromagnetic wave which is dentified as light.'

Next expected EM phase is fields be taken more primitive than material sources. Physical laws derived from nonlinear fields became a next understanding. Einstein general relativity equations gave a first argument, by starting a nonlinear field theory [25]. Various nonlinear EM models are being proposed at literature. A nonlinear electromagnetism physicality where instead of charges and magnets, fields work as the own sources is expected. Faraday lines of force be rewritten through nonlinearity.

Under this perspective, eq.(1.1) introduces a non-linear electromagnetism where the fields are the own sources, expressing 
that electromagnetic is more than a phenomena related to electric charge and spin. A nonlinear approach is derived by intepreting on electric charge physics behaviour. While Maxwell studied the electric charge behaviour macroscopically through their distributions, elementary particles physics considered its physics microscopically. Three consequences happened. They are the electric charge quantization, conducted by particles with different flavours and spins and manifested through a tryad $\{+,-, 0\}$.

A new aspect to be explored is that elementary particles physics reactions are showing on charges $\{+,-, 0\}$ converting in each order. It shows that the Maxwell photon is not enough for describing the microscopic electric charge physics. It is necessary four bosons fields in order to intermediate the charge exchange $\triangle Q=0, \pm 1$. As consequence the physics of electric charge is extended and nonlinear fields working as own sources are introduced. Since 1930 decade different attempts have been developed. Effective theories based on Euler-Heisenberg and Born-Infeld approaches have been developed. However, historically, there is a missing EM to be understood. Our answer is to consider fundamental nonlinear EM energy based on three electric charges transformations to be considered.

The four EM fields not only expands the meaning of electric charge behavior in terms for conservation, conduction, transmission, interaction but also develops a new EM flux. Maxwell provides just the EM flux simplest case. It is given by $\vec{E}-\vec{B}$ and as EM domain $\mathcal{L}=F_{\mu \nu}^{2}$. The Four Bosons EM flux enlarges the Maxwell flux. A microscopic electromagnetism is developed, it works out with a charges set $\{+, 0,-\}$ and introduces a fields set $\left\{A_{\mu}, U_{\mu}, V_{\mu}^{ \pm}\right\}$. EM is enlarged. It yields a physics not depending on external sources, on permissibility $\epsilon$ and permeability $\mu$ constants, and with coupling constants beyond electric charge.

Interlaces the photon with massive photon and charged photons. New elements as fields conglomerates, granular and collective fields strengths, non-linear currents are performed. They also constituted an EM with different interconnected sectors. Each one is constituted by gauge invariant and real regions inserted by eq.(2.1) Lagrangian. Each one of them flows an own EM energy connected with others carrying spin- 1 and spin-0 and by different pieces which are called here as EM domains. Interdependent physical regions where each one contains its own physicality. Carrying its own energy and momentum but under overall conservation laws.

Three results are derived. They are on electric charges, fields as sources and potentials fields physicality. Electric charge is more than being expressed by Coulomb law, conserved entity and transmitted by photon. The novelty it is preceded by an EM energy being derived from the tryad $\{+,-, 0\}$ interchange. A new charge physics is obtained. It yields on electric charge physics carried by four bosons and with a conservation law expressed by Noether theorem with effects beyond QED. It provides the zero charge acting on the electromagnetic manifestation.

A second consequence is on nonlinear fields sources. $(4+4)$ sources are generated from equations of motion fro spin-1 and spin- 0 . Plus one for collective fields through Bianchi identities; 2 energy-momentum conservation laws; 3 Noether identities; 1 angular momentum conservation. In total 15 conservation laws and respective fields sources are obtained.

A third consequence is on potential fields. Not being gauge invariant they have not been considered as physical entities. Heaviside has stated: ' $\vec{A}$ and its scalar potential parasite $\phi$ sometimes causing great mathematical complexity and indistinctiveness; and it is for pratical reasons best to murder the whole lot or, at any rate, merely employ them as subsidiary functions...'. As counterpart the role of vector potential fields has been intensively discussed. Quantum Mechanics considers $e \vec{A}$ as linear momentum and $\phi$ as potential energy. They are explicity incorporated in equations.

Concluding, Maxwell equations should be considered just as part of the electromagnetic phenomena. It discovered two postulates which are light invariance and electric charge conservation. Nevertheless elementary particles physics shows 
the electric charge behaviour as a set $\{+,-, 0\}$. A microscopic systemic electric charge is constituted based on Maxwell postulates. A nonlinear four bosons EM is introduced. Its corresponding conservation laws are showing that there is an EM flux beyond electric charge. There is showing a new EM flux and conservation laws to be explored.

\section{Appendix A. Collective Fields}

Alongside the usual granular fields, new collective fields appear, defined by:

$$
\begin{aligned}
& z_{\mu \nu}=\gamma_{I J} G_{\mu}^{I} G_{\nu}^{J} \\
& =\gamma_{11} G_{\mu}^{1} G_{\nu}^{1}+\gamma_{12} G_{\mu}^{1} G_{\nu}^{2}+\gamma_{13} G_{\mu}^{1} G_{\nu}^{3}+\gamma_{14} G_{\mu}^{1} G_{\nu}^{4}+ \\
& +\gamma_{21} G_{\mu}^{2} G_{\nu}^{1}+\gamma_{22} G_{\mu}^{2} G_{\nu}^{2}+\gamma_{23} G_{\mu}^{2} G_{\nu}^{3}+\gamma_{24} G_{\mu}^{2} G_{\nu}^{4}+ \\
& +\gamma_{31} G_{\mu}^{3} G_{\nu}^{1}+\gamma_{32} G_{\mu}^{3} G_{\nu}^{2}+\gamma_{33} G_{\mu}^{3} G_{\nu}^{3}+\gamma_{34} G_{\mu}^{3} G_{\nu}^{4}+ \\
& +\gamma_{41} G_{\mu}^{4} G_{\nu}^{1}+\gamma_{42} G_{\mu}^{4} G_{\nu}^{2}+\gamma_{43} G_{\mu}^{4} G_{\nu}^{3}+\gamma_{44} G_{\mu}^{4} G_{\nu}^{4}
\end{aligned}
$$

The collective antisymmetric fields are

$$
z_{[\mu \nu]}=\left(\begin{array}{cccc}
0 & z^{[1,2]} & z^{[1,+]} & z^{[1,-]} \\
-z^{[1,2]} & 0 & z^{[2,+]} & z^{[2,-]} \\
-z^{[+, 1]} & -z^{[+, 2]} & 0 & z^{[+,-]} \\
-z^{[-, 1]} & -z^{[-, 2]} & -z^{[-,+]} & 0
\end{array}\right)
$$

there are also 10 symmetric collective fields,

$$
z_{(\mu \nu)}=\left(\begin{array}{llll}
z^{(1,1)} & z^{(1,2)} & z^{(1,+)} & z^{(1,-)} \\
z^{(1,2)} & z^{(2,2)} & z^{(2,+)} & z^{(2,-)} \\
z^{(1,+)} & z^{(2,+)} & z^{(+,+)} & z^{(+,-)} \\
z^{(1,-)} & z^{(2,-)} & z^{(+,-)} & z^{(-,-)}
\end{array}\right)
$$


Considering the fields set $\left\{A_{\mu}, U \mu, V_{\mu}^{ \pm}\right\}$one gets the following group of collective fields,

$$
\begin{aligned}
& \stackrel{(11)}{z} \mu \nu \equiv \gamma_{(11)} A^{\mu} A^{\nu}, \quad \stackrel{(22)}{z}^{\mu \nu} \equiv \gamma_{(22)} U^{\mu} U^{\nu}, \quad \stackrel{(12)}{z} \mu \nu \equiv \gamma_{(12)} A^{\mu} U^{\nu}, \\
& \stackrel{(21)}{z}_{\mu \nu} \equiv \gamma_{(21)} U^{\mu} A^{\nu} \quad \stackrel{(11)}{z}_{\mu}^{\mu}=\gamma_{(11)} A_{\mu} A^{\mu}, \quad \stackrel{(22)}{z}_{\mu}=\gamma_{(22)} U_{\mu} U^{\mu} \text {, } \\
& \stackrel{(12)}{z_{\mu}}=\gamma_{(12)} A_{\mu} U^{\mu}, \quad{ }_{z}^{[12]} \mu \nu \equiv \gamma_{[12]} A^{\mu} U^{\nu}, \quad{ }_{z}^{[21]} \mu \nu \equiv \gamma_{[21]} U^{\mu} A^{\nu},
\end{aligned}
$$

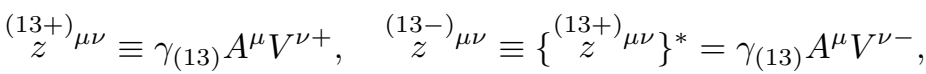

$$
\begin{aligned}
& \stackrel{(14+)}{z} \mu \nu \equiv \gamma_{(14)} A^{\mu} V^{\nu+}, \quad \underset{z}{(14-)} \mu \nu \equiv\left\{\stackrel{(14+)}{z}{ }^{\mu \nu}\right\}^{*}=\gamma_{(14)} A^{\mu} V^{\nu-}, \\
& { }_{z}^{[13+]}{ }_{\mu \nu} \equiv \gamma_{[13]} A^{\mu} V^{\nu+}, \quad{ }_{z}^{[13-]_{\mu \nu}} \equiv\left\{\stackrel{[13+]}{z}_{\mu \nu}\right\}^{*}=\gamma_{[13]} A^{\mu} V^{\nu-}, \\
& \stackrel{[14+]}{z}_{\mu \nu} \equiv \gamma_{[14]} A^{\mu} V^{\nu+}, \quad{ }_{z}^{[14-]_{\mu \nu}} \equiv\left\{{\stackrel{[14+]}{z}]_{\mu \nu}}^{*}=\gamma_{[14]} A^{\mu} V^{\nu-},\right. \\
& \stackrel{(23+)}{z} \mu \nu \equiv \gamma_{(23)} U^{\mu} V^{\nu+}, \quad \underset{z}{(23-)} \mu \nu \equiv\{\stackrel{(23+)}{z} \mu \nu\}^{*}=\gamma_{(23)} U^{\mu} V^{\nu-}, \\
& \stackrel{(24+)}{z} \mu \nu \equiv \gamma_{(24)} U^{\mu} V^{\nu+}, \quad \underset{z}{(24-)} \mu \nu \equiv\{\stackrel{(24+)}{z} \mu \nu\}^{*}=\gamma_{(24)} U^{\mu} V^{\nu-}, \\
& \left.\left.{ }_{z}^{[23+]}\right]_{\mu \nu} \equiv \gamma_{[23]} U^{\mu} V^{\nu+}, \quad{ }_{z}^{[23-]} \mu{ }^{[23+]}{ }^{[2 \mu}\right\}^{*}=\gamma_{[23]} U^{\mu} V^{\nu-}, \\
& { }_{z}^{[24+]} \mu \nu \equiv \gamma_{[24]} U^{\mu} V^{\nu+}, \quad{ }_{z}^{[24-]_{\mu \nu}} \equiv\{\underset{z}{[24+]} \mu \nu\}^{*}=\gamma_{[24]} U^{\mu} V^{\nu-}, \\
& { }^{+} z^{3} \mu \nu \equiv \gamma_{(33)} V^{\mu+} V^{\nu-}, \quad{ }_{z}^{+3} \mu \nu \equiv\left\{{ }^{+-3}{ }^{3 \nu}\right\}^{*}=\gamma_{(33)} V^{\mu-} V^{\nu+}, \\
& { }^{+} \bar{z}^{4} \mu \nu \equiv \gamma_{(44)} V^{\mu+} V^{\nu-}, \quad{ }_{z}^{+4} \mu \nu \equiv\left\{{ }^{+-4} z^{4 \nu}\right\}^{*}=\gamma_{(44)} V^{\mu-} V^{\nu+} \text {, } \\
& { }_{z}^{(+-)} \mu \nu \equiv-i \gamma_{(34)} V^{\mu+} V^{\nu-}, \quad{ }_{z}^{(-+)} \mu \nu \equiv\left\{{ }_{z}^{(+-)} \mu \nu\right\}^{*}=i \gamma_{(34)} V^{\mu-} V^{\nu+} \text {, } \\
& { }_{z}^{[+-]_{\mu \nu}} \equiv-i \gamma_{[34]} V^{\mu+} V^{\nu-}, \quad{ }_{z}^{[-+]} \mu \nu \equiv\left\{{ }^{[+-]_{\mu \nu}}\right\}^{*}=i \gamma_{[34]} V^{\mu-} V^{\nu+} \text {, } \\
& \stackrel{(+1)}{z} \mu \nu \equiv\left(\gamma_{(13)}+i \gamma_{(14)}\right) A^{\mu} V^{\nu+}, \quad \stackrel{(-1)}{z} \mu \nu \equiv\{\stackrel{(+1)}{z} \mu \nu\}^{*}, \\
& \stackrel{(+2)}{z}^{\mu \nu} \equiv\left(\gamma_{(23)}+i \gamma_{(24)}\right) U^{\mu} V^{\nu+}, \quad \stackrel{(-2)}{z} \mu \nu \equiv\{\stackrel{(+2)}{z} \mu \nu\}^{*}, \\
& \stackrel{[+1]}{z} \mu \nu \equiv\left(\gamma_{[13]}+i \gamma_{[14]}\right) A^{\mu} V^{\nu+}, \quad \stackrel{[-1]}{z} \mu \nu \equiv\left\{{ }_{z}^{[+1]} \mu \nu\right\}^{*}, \\
& \stackrel{[+2]}{z}^{\mu \nu} \equiv\left(\gamma_{[23]}+i \gamma_{[24]}\right) U^{\mu} V^{\nu+}, \quad \stackrel{[-2]}{z} \mu \nu \equiv\{\stackrel{[+2]}{z} \mu \nu\}^{*} \text {. }
\end{aligned}
$$

Similarly, one adds the $\omega$ collective fields associated to $\tau_{i j}$

$$
\begin{aligned}
& \stackrel{(11)}{\omega}_{\alpha} \equiv \tau_{(11)} A^{\alpha} A_{\alpha}, \quad \stackrel{(22)}{\omega}_{\alpha} \equiv \tau_{(22)} U^{\alpha} U_{\alpha}, \quad \stackrel{(12)}{\omega}_{\alpha} \equiv \tau_{(12)} A^{\alpha} U_{\alpha}, \\
& { }^{+-3^{3}}{ }_{\alpha} \equiv \tau_{(33)} V^{\alpha+} V_{\alpha-}, \quad \stackrel{(+1)}{\omega}_{\alpha} \equiv\left(\tau_{(13)}+i \tau_{(14)}\right) A^{\alpha} V_{\alpha}^{+},
\end{aligned}
$$

Rewriting $z_{[\mu \nu]}$ and $z_{(\mu \nu)}$, one gets

$$
\begin{aligned}
& z_{[\mu \nu]}=\gamma_{[12]}\left(A_{\mu} U_{\nu}-A_{\nu} U_{\mu}\right)+\frac{1}{\sqrt{2}}\left(\gamma_{[13]}+i \gamma_{[14]}\right)\left(A_{\mu} V_{\nu}^{+}-A_{\nu} V_{\mu}^{+}\right)+ \\
& +\frac{1}{\sqrt{2}}\left(\gamma_{[13]}-i \gamma_{[14]}\right)\left(A_{\mu} V_{\nu}^{-}-A_{\nu} V_{\mu}^{-}\right)+\frac{1}{\sqrt{2}}\left(\gamma_{[23]}+i \gamma_{[24]}\right)\left(U_{\mu} V_{\nu}^{+}-U_{\nu} V_{\mu}^{+}\right)+ \\
& +\frac{1}{\sqrt{2}}\left(\gamma_{[23]}+i \gamma_{[24]}\right)\left(U_{\mu} V_{\nu}^{-}-U_{\nu} V_{\mu}^{-}\right)-\gamma_{[34]}\left(V_{\mu}^{+} V_{\nu}^{-}-V_{\mu}^{-} V_{\nu}^{+}\right)
\end{aligned}
$$

or

$$
z_{[\mu \nu]}=2 \stackrel{[12]}{z}_{[\mu \nu]}+\sqrt{2} \stackrel{[+1]}{z}_{[\mu \nu]}+\sqrt{2} \stackrel{[-1]}{z}_{[\mu \nu]}+2 \stackrel{[+2]}{z}_{[\mu \nu]}+\sqrt{2} \stackrel{[-2]}{z}_{[\mu \nu]}+\stackrel{[+-]}{z}_{[\mu \nu]}
$$


And,

$$
\begin{aligned}
& z_{(\mu \nu)}=\gamma_{11} A_{\mu} A_{\nu}+\gamma_{22} U_{\mu} U_{\nu}+\frac{1}{2}\left(\gamma_{33}-\gamma_{44}\right)\left(V_{\mu}^{+} V_{\nu}^{-}+V_{\mu}^{-} V_{\nu}^{+}\right)+ \\
& +\frac{1}{2}\left(\gamma_{33}+\gamma_{44}\right)\left(V_{\mu}^{+} V_{\nu}^{-}+V_{\mu}^{-} V_{\nu}^{+}\right)+\gamma_{(12)}\left(A_{\mu} U_{\nu}+U_{\mu} A_{\nu}\right)+ \\
& +\frac{1}{\sqrt{2}}\left(\gamma_{(13)}+i \gamma_{(14)}\right)\left(A_{\mu} V_{\nu}^{+}+A_{\nu} V_{\mu}^{+}\right)+\frac{1}{\sqrt{2}}\left(\gamma_{(13)}-i \gamma_{(14)}\right)\left(A_{\mu} V_{\nu}^{-}+A_{\nu} V_{\mu}^{-}\right)+ \\
& +\frac{1}{\sqrt{2}}\left(\gamma_{(23)}+i \gamma_{(24)}\right)\left(U_{\mu} V_{\nu}^{+}+U_{\nu} V_{\mu}^{+}\right)+\frac{1}{\sqrt{2}}\left(\gamma_{(23)}-i \gamma_{(24)}\right)\left(U_{\mu} V_{\nu}^{-}+U_{\nu} V_{\mu}^{-}\right)+ \\
& +i \gamma_{(34)}\left(V_{\mu}^{+} V_{\nu}^{-}+V_{\mu}^{-} V_{\nu}^{+}\right)
\end{aligned}
$$

\section{Appendix B. Hamiltonian Positivity}

Considering the hamiltonian correspondent to the kinetic symmetric sector, one gets

$$
\begin{aligned}
& U_{\mathrm{KS}}=b_{(11)}\left(-\frac{3}{2} S_{A}^{2}+\vec{S}_{A} \cdot \vec{S}_{A}+\frac{1}{2} \stackrel{\leftrightarrow}{S}_{A} \cdot \stackrel{\leftrightarrow}{S}_{A}\right)+ \\
& +b_{(22)}\left(-\frac{3}{2} S_{U}^{2}+\vec{S}_{U} \cdot \vec{S}_{U}+\frac{1}{2} \stackrel{\leftrightarrow}{S}_{U} \cdot \stackrel{\leftrightarrow}{S}_{U}\right)+ \\
& +b_{(33)}\left(-3 S_{+} S_{-}+2 \vec{S}_{+} \cdot \vec{S}_{-}+\stackrel{\leftrightarrow}{S}_{+} \cdot \stackrel{\leftrightarrow}{S}_{-}\right)+ \\
& +c_{(11)}\left(-\frac{3}{2} S_{A}^{2}+S_{A} S_{i A}^{i}+\frac{1}{2} S_{i A}^{i 2}\right)+ \\
& +c_{(22)}\left(-\frac{3}{2} S_{U}^{2}+S_{U} S_{i U}^{i}+\frac{1}{2} S_{i U}^{i 2}\right)+ \\
& +c_{(33)}\left(-3 S_{+} S_{-}+S_{+} S_{-i}^{i}+S_{-} S_{+i}^{i}+S_{i+}^{i} S_{j-}^{j}\right)+ \\
& +\frac{1}{4} \xi_{(11)}\left(-\frac{3}{2} S_{A}^{2}+S_{A} S_{i A}^{i}+\frac{1}{2} S_{i A}^{i 2}\right)+ \\
& +\frac{1}{4} \xi_{(22)}\left(-\frac{3}{2} S_{U}^{2}+S_{U} S_{i U}^{i}+\frac{1}{2} S_{i U}^{i 2}\right)+ \\
& +\frac{1}{4} \xi_{(12)}\left(-3 S_{A} S_{U}+S_{A} S_{U i}^{i}+S_{U} S_{A i}^{i}+S_{i A}^{i} S_{j U}^{j}\right)+ \\
& +\frac{1}{4} \xi_{(33)}\left(-3 S_{+} S_{-}+S_{+} S_{-i}^{i}+S_{-} S_{+i}^{i}+S_{i+}^{i} S_{j-}^{j}\right)+
\end{aligned}
$$

To assure the positivity of the Hamiltonian, we can use the gauge fixing term and stablish the following relations:

$$
\begin{aligned}
& \xi_{(11)}=4\left(b_{(11)}+c_{(11)}\right) \\
& \xi_{(22)}=4\left(b_{(22)}+c_{(22)}\right) \\
& \xi_{(33)}=4\left(b_{(33)}+c_{(33)}\right) \\
& \xi_{(12)}=0
\end{aligned}
$$

Obtaining the positive expression: 


$$
\begin{aligned}
& U=b_{(11)}\left(\vec{S}_{A} \cdot \vec{S}_{A}+\frac{1}{2} \stackrel{\leftrightarrow}{S}_{A} \cdot \stackrel{\leftrightarrow}{S}_{A}\right)+ \\
& +b_{(22)}\left(\vec{S}_{U} \cdot \vec{S}_{U}+\frac{1}{2} \stackrel{\leftrightarrow}{S}_{U} \cdot \overleftrightarrow{S}_{U}\right)+ \\
& +b_{(33)}\left(2 \vec{S}_{+} \cdot \vec{S}_{-}+\stackrel{\leftrightarrow}{S}_{+} \cdot \stackrel{\leftrightarrow}{S}_{-}\right)+ \\
& +c_{(11)}\left(S_{A} S_{i A}^{i}+\frac{1}{2} S_{i A}^{i 2}\right)+ \\
& +c_{(22)}\left(S_{U} S_{i U}^{i}+\frac{1}{2} S_{i U}^{i 2}\right)+ \\
& +c_{(33)}\left(S_{+} S_{-i}^{i}+S_{-} S_{+i}^{i}+S_{i+}^{i} S_{j-}^{j}\right)+ \\
& +\frac{1}{4} \xi_{(11)}\left(S_{A} S_{i A}^{i}+\frac{1}{2} S_{i A}^{i 2}\right)+ \\
& +\frac{1}{4} \xi_{(22)}\left(S_{U} S_{i U}^{i}+\frac{1}{2} S_{i U}^{i 2}\right)+ \\
& +\frac{1}{4} \xi_{(12)}\left(S_{A} S_{U i}^{i}+S_{U} S_{A i}^{i}+S_{i A}^{i} S_{j U}^{j}\right)+ \\
& +\frac{1}{4} \xi_{(33)}\left(S_{+} S_{-i}^{i}+S_{-} S_{+i}^{i}+S_{i+}^{i} S_{j-}^{j}\right)+
\end{aligned}
$$

\section{References}

[1] J. C. Maxwell, London Edinburgh Dublin Phys. Mag and J.Sc. Series IV, 21, 161 (196)

[2] J. Helayel Neto, http://www.professorglobal.com.br/fisica-pos-graduacao-2017/eletromagnetismo/videos

[3] J. Plebanski. 'Lectures On Non-Linear Electrodynamics' (1970); G. Boillat, JMP, Vol 11, N3, (1970); G.Boillat, Lettere Al Nuovo Cimento, Vol4, N7 (1972)

[4] J. Chauca, R.Doria, I. Soares JAP Vol 10 No1 2605 (2015); J. Chauca, R. Doria, and I. Soares, Electric charge transmission through four bosons. JAP, 13(1):4535-4592, 2017.; J. Chauca, R. Doria, W.Soares, Non-Linear abelian gauge model, AIP Conf. Proc. 1483, 342-351 (2012)

[5] Y. Aharonov and D. Bohm, Significance of electromagnetic potentials in the quantum theory, Phys. Rev. 115 (1959) 48.

[6] J. Schwinger, Selected Papers on Quantum Electrodynamics Dowe Publications (1958); P.A.M Dirac. A theory of electrons and protons PRSL, 26(1930) 361; P.A.M Dirac. On quantum electrodynamics. Physikdische Zeitschrift der Sowieturian 2(1933) 468

[7] A Compton, The magnetic electron, Jour. Franklin Inst. 192:2 (1921) 145

[8] Gerlach, W.; Stern, O. (1922). "Der experimentelle Nachweis der Richtungsquantelung im Magnetfeld". Zeitschrift für Physik. 9: 349-352; Gerlach, W.; Stern, O. (1922). "Das magnetische Moment des Silberatoms". Zeitschrift für Physik. 9: 353-355; Gerlach, W.; Stern, O. (1922). "Der experimentelle Nachweis des magnetischen Moments des Silberatoms". Zeitschrift für Physik. 8: 110-111. 
[9] P.A.M. Dirac, The quantum theory of the electron-I, Proc. R. Soc. Lond. A 117 (1928) 610; The quantum theory of the electron II, Proc. R. Soc. Lond. A 118 (1928)

[10] H. Ohanian, And what is spin?, Am. J. Phys. 54,6 (1986)

[11] R. Doria and I. Soares, Spin Valued Four Bosons Electromagnetism, submitted paper (2021)

[12] E.J. Konopinski, what the vector potential describes, Am. J. Phys 46, 499-502 (1978); A.C.T $W_{\mu}$ and C.N.Yang, Evolution of the concept of the vector potential in the description of fundamental interactions, Int.J.Mod.Phys. A 21, 32335-3277 (2006). Y. Aharonov and A. Casher, Phys. Rev. Lett. 53 (1984) 319; Y. Aharonov, P. Pearle and L. Vaidman, Phys. Rev. A37 (1988) 4052

[13] G. Breit and John A. Wheeler. Collison of two light quanta. Phys. Rev., 46:1087-1091, Dec 1934.

[14] S.L. Adler, Ann. Phys 67 (1971)

[15] M. Delbruck, Zeits. Physik PR 36 (1930) 1590;84 (1933) 144

[16] P.A.M Dirac, Quantised singularities in the electromagnetic field, PRSL A 133 (1931) 60.

[17] G.'t Hooft, Nucl.Phys.B79 (1974) 276; A.M. Polyakov, JETP Letters 20(1974)

[18] W. Heisenberg, H. Euler, Zeits. Physik 98,714(1936); H. Euler, Ann der Phys. 418(1936); H. Kleivert, arXiv: physics/0605038 [phys.hist-ph] (2006)

[19] M. Born and L. Infeld. Electromagnetic mass. Nature, 132:970, 1932.; M. Born and L. Infeld. Foundations of the new field theory. Proceedings of the Royal Society of London A: Mathematical, Physical and Engineering Sciences, 144(852):425-451, 1934 .

[20] H,J,W Müller-Kirsten, Electrodynamics: An Introduction including Quantum Effects World Scientific

[21] E. Noether, Nach. König. der Gevel. der Wissen. du Göttingen, Math.-Phys.Klasse (1918) 235; B. Berchel, D. Malterre, E. Medina, Gauge transformations and conserved quantities in classical and quantum mechanics, Am. J. Phys. 84 (2016); doi: 101119/1. 4955153

[22] R. Doria, Physics of Light, AIP Conf. Proc. 1483, 35-117 (2012)

[23] J. Chauca, R. Doria, W.Soares, A whole origin for polarization and magnetization, AIP Conf. Proc. 1483, 395-399 (2012); F.S Johnson, B.L. Cragin and R.R. Hodges, Electromagnetic momentum density and the Poynting vector in static fields, Am. J. Phys. 62, 33-41 (1994); M.G.Calkin, Linear momentum of quasiestatic electromagnetic fields, Am. J. Phys. 34, 921-925 (1966)

[24] M. Faraday, Experimental Researches in Electricity, 3 vols, 1839,1844, 1855. Taylor \& T. Francis, vols 1 and 3

[25] A.Einstein, The fields equations of gravitation, Preussische Akademie der Wissenschaften, Sitzungsberichte, 1915 (part 2), 831 